\title{
Fast and Compact Self-Stabilizing Verification, Computation, and Fault Detection of an MST
}

Amos Korman *

\author{
Shay Kutten ${ }^{\dagger}$
}

\author{
Toshimitsu Masuzawa ${ }^{\ddagger}$
}

\begin{abstract}
This paper demonstrates the usefulness of distributed local verification of proofs, as a tool for the design of self-stabilizing algorithms. In particular, it introduces a somewhat generalized notion of distributed local proofs, and utilizes it for improving the time complexity significantly, while maintaining space optimality. As a result, we show that optimizing the memory size carries at most a small cost in terms of time, in the context of Minimum Spanning Tree (MST). That is, we present algorithms that are both time and space efficient for both constructing an MST and for verifying it. This involves several parts that may be considered contributions in themselves.

First, we generalize the notion of local proofs, trading off the time complexity for memory efficiency. This adds a dimension to the study of distributed local proofs, which has been gaining attention recently. Specifically, we design a (self-stabilizing) proof labeling scheme which is memory optimal (i.e., $O(\log n)$ bits per node), and whose time complexity is $O\left(\log ^{2} n\right)$ in synchronous networks, or $O\left(\Delta \log ^{3} n\right)$ time in asynchronous ones, where $\Delta$ is the maximum degree of nodes. This answers an open problem posed by Awerbuch and Varghese (FOCS 1991). We also show that $\Omega(\log n)$ time is necessary, even in synchronous networks. Another property is that if $f$ faults occurred, then, within the required detection time above, they are detected by some node in the $O(f \log n)$ locality of each of the faults.

Second, we show how to enhance a known transformer that makes input/output algorithms self-stabilizing. It now takes as input an efficient construction algorithm and an efficient self-stabilizing proof labeling scheme, and produces an efficient self-stabilizing algorithm. When used for MST, the transformer produces a memory optimal self-stabilizing algorithm, whose time complexity, namely, $O(n)$, is significantly better even than that of previous algorithms. (The time complexity of previous MST algorithms that used $\Omega\left(\log ^{2} n\right)$ memory bits per node was $O\left(n^{2}\right)$, and the time for optimal space algorithms was $O(n|E|)$.) Inherited from our proof labelling scheme, our self-stabilising MST construction algorithm also has the following two properties: (1) if faults occur after the construction ended, then they are detected by some nodes within $O\left(\log ^{2} n\right)$ time in synchronous networks, or within $O\left(\Delta \log ^{3} n\right)$ time in asynchronous ones, and (2) if $f$ faults occurred, then, within the required detection time above, they are detected within the $O(f \log n)$ locality of each of the faults. We also show how to improve the above two properties, at the expense of some increase in the memory.
\end{abstract}

Keywords: Distributed network algorithms, Locality, Proof labels, Minimum spanning tree, Distributed property verification, Self-stabilization, Fast fault detection, Local fault detection.

\footnotetext{
${ }^{*}$ CNRS and Univ. Paris Diderot, Paris, 75013, France. E-mail: Amos.Korman@liafa.jussieu. fr. Supported in part by a France-Israel cooperation grant ("Mutli-Computing" project) from the France Ministry of Science and Israel Ministry of Science, by the ANR projects ALADDIN and PROSE, and by the INRIA project GANG.

${ }^{\dagger}$ Information Systems Group, Faculty of IE\&M, The Technion, Haifa, 32000 Israel. E-mail: my last name at ie dot technion dot ac dot il. Supported in part by a France-Israel cooperation grant ("Mutli-Computing" project) from the France Ministry of Science and Israel Ministry of Science, by a grant from the Israel Science Foundation, and by the Technion funds for security research.

${ }^{\ddagger}$ Graduate School of Information Science and Technology, Osaka University, 1-5 Yamadaoka, Suita, Osaka, 565-0871, Japan. E-mail: masuzawa@ist. osaka-u.ac. jp. Supported in part by JSPS Grant-in-Aid for Scientific Research ((B)26280022).
} 


\section{Introduction}

\subsection{Motivation}

In a non-distributed context, solving a problem is believed to be, sometimes, much harder than verifying it (e.g., for NP-Hard problems). Given a graph $G$ and a subgraph $H$ of $G$, a task introduced by Tarjan [65] is to check whether $H$ is a Minimum Spanning Tree (MST) of $G$. This non-distributed verification seems to be just slightly easier than the non-distributed computation of an MST. In the distributed context, the given subgraph $H$ is assumed to be represented distributively, such that each node stores pointers to (some of) its incident edges in $H$. The verification task consists of checking whether the collection of pointed edges indeed forms an MST, and if not, then it is required that at least one node raises an alarm. It was shown recently that such an MST verification task requires the same amount of time as the MST computation [26, 53]. On the other hand, assuming that each node can store some information, i.e., a label, that can be used for the verification, the time complexity of an MST verification can be as small as 1, when using labels of size $\Theta\left(\log ^{2} n\right)$ bits per node [54, 55], where $n$ denotes the number of nodes. To make such a proof labeling scheme a useful algorithmic tool, one needs to present a marker algorithm for computing those labels. One of the contributions of the current paper is a time and memory efficient marker algorithm.

Every decidable graph property (not just an MST) can be verified in a short time given large enough labels [55]. A second contribution of this paper is a generalization of such schemes to allow a reduction in the memory requirements, by trading off the locality (or the time). In the context of MST, yet another (third) contribution is a reduced space proof labeling scheme for MST. It uses just $O(\log n)$ bits of memory per node (asymptotically the same as the amount of bits needed for merely representing distributively the MST). This is below the lower bound of $\Omega\left(\log ^{2} n\right)$ of [54]. The reason this is possible is that the verification time is increased to $O\left(\log ^{2} n\right)$ in synchronous networks and to $O\left(\Delta \log ^{3} n\right)$ in asynchronous ones, where $\Delta$ is the maximum degree of nodes. Another important property of the new scheme is that any fault is detected rather close to the node where it occurred. Interestingly, it turns out that a logarithmic time penalty for verification is unavoidable. That is, we show that $\Omega(\log n)$ time for an MST verification scheme is necessary if the memory size is restricted to $O(\log n)$ bits, even in synchronous networks. (This, by the way, means that a verification with $O(\log n)$ bits, cannot be silent, in the sense of [33]; this is why they could not be of the kind introduced in [55]).

Given a long enough time, one can verify $T$ by recomputing the MST. An open problem posed by Awerbuch and Varghese [15] is to find a synchronous MST verification algorithm whose time complexity is smaller than the MST computation time, yet with a small memory. This problem was introduced in [15] in the context of selfstabilization, where the verification algorithm is combined with a non-stabilizing construction protocol to produce a stabilizing protocol. Essentially, for such purposes, the verification algorithm repeatedly checks the output of the non-stabilizing construction protocol, and runs the construction algorithm again if a fault at some node is detected. Hence, the construction algorithm and the corresponding verification algorithm are assumed to be designed together. This, in turn, may significantly simplify the checking process, since the construction algorithm may produce output variables (labels) on which the verification algorithm can later rely. In this context, the above mentioned third contribution solves this open problem by showing an $O\left(\log ^{2} n\right)$ time penalty (in synchronous networks) when using optimal $O(\log n)$ memory size for the MST verification algorithm. In contrast, if we study MST construction instead of MST verification, time lower bounds which are polynomial in $n$ for MST construction follow from [58, 62] (even for constant diameter graphs).

One known application of some methods of distributed verification is for general transformers that transform non-self-stabilizing algorithms to self-stabilizing ones. The fourth contribution of this paper is an adaptation of the transformer of [15] such that it can transform algorithms in our context. That is, while the transformer of [15] requires that the size of the network and its diameter are known, the adapted one allows networks of 
unknown size and diameter. Also, here, the verification method is a proof labeling scheme whose verifier part is self-stabilizing. Based on the strength of the original transformer of [15] (and that of the companion paper [13] it uses), our adaptation yields a result that is rather useful even without plugging in the new verification scheme. This is demonstrated by plugging in the proof labeling schemes of [54, 55], yielding an algorithm which already improves the time of previous $O\left(\log ^{2} n\right)$ memory self-stabilizing MST construction algorithm [17], and also detects faults using 1 time and at distance at most $f$ from each fault (if $f$ faults occurred).

Finally, we obtain an optimal $O(\log n)$ memory size, $O(n)$ time asynchronous self-stabilizing MST construction algorithm. The state of the art time bound for such optimal memory algorithms was $O(n|E|)[18,48]$. In fact, our time bound improves significantly even the best time bound for algorithms using polylogarithmic memory, which was $O\left(n^{2}\right)$ [17].

Moreover, our self-stabilizing MST algorithm inherits two important properties from our verification scheme, which are: (1) the time it takes to detect faults is small: $O\left(\log ^{2} n\right)$ time in a synchronous network, or $O\left(\Delta \log ^{3} n\right)$ in an asynchronous one; and (2) if some $f$ faults occur, then each fault is detected within its $O(f \log n)$ neighbourhood. Intuitively, a short detection distance and a small detection time may be helpful for the design of local correction, for fault confinement, and for fault containment algorithms [43, 16]. Those notions were introduced to combat the phenomena of faults "spreading" and "contaminating" non-faulty nodes. For example, the infamous crash of the ARPANET (the predecessor of the Internet) was caused by a fault in a single node. This caused old updates to be adopted by other nodes, who then generated wrong updates affecting others [59]. This is an example of those non-faulty nodes being contaminated. The requirement of containment [43] is that such a contamination does not occur, or, at least, that it is contained in a small radius around the faults. The requirement of confinement [16] allows the contamination of a state of a node, as long as this contamination is not reflected in the output (or the externally visible actions) of the non-faulty nodes. Intuitively, if the detection distance is short, non-faulty nodes can detect the faults and avoid being contaminated.

\subsection{Related work}

The distributed construction of an MST has yielded techniques and insights that were used in the study of many other problems of distributed network protocols. It has also become a standard to check a new paradigm in distributed algorithms theory. The first distributed algorithm was proposed by [25], its complexity was not analyzed. The seminal paper of Gallager, Humblet, and Spira presented a message optimal algorithm that used $O(n \log n)$ time, improved by Awerbuch to $O(n)$ time [40, 9], and later improved in [57, 41] to $O\left(D+\sqrt{n} \log ^{*} n\right)$, where $D$ is the diameter of the network. This was coupled with an almost matching lower bound of $\Omega(D+\sqrt{n})[62]$.

Proof labeling schemes were introduced in [55]. The model described therein assumes that the verification is restricted to 1 unit of time. In particular, a 1 time MST verification scheme was described there using $O\left(\log ^{2} n\right)$ bits per node. This was shown to be optimal in [54]. In [46], Göös and Suomela extend the notion of proof labeling schemes by allowing constant time verification, and exhibit some efficient proof labeling schemes for recognizing several natural graph families. In all these schemes, the criterion to decide failure of a proof (that is, the detection of a fault) is the case that at least one node does not manage to verify (that is, detects a fault). The global state passes a proof successfully if all the nodes verify successfully. This criterion for detection (or for a failure to prove) was suggested by [2, 3] in the contexts of self stabilization, and used in self stabilization (e.g. $[13,14,15])$ as well as in other other contexts [60].

Self-stabilization [29] deals with algorithms that must cope with faults that are rather severe, though of a type that does occur in reality [50]. The faults may cause the states of different nodes to be inconsistent with each other. For example, the collection of marked edges may not be an MST.

Table 1.2 summarizes the known complexity results for self stabilizing MST construction algorithms. The 
first several entrees show the results of using (to generate an MST algorithm automatically) the known transformer of Katz and Perry [52], that extends automatically non self stabilizing algorithms to become self stabilizing. The transformer of Katz and Perry [52] assumes a leader whose memory must hold a snapshot of the whole network. The time of the resulting self-stabilizing MST algorithm is $O(n)$ and the memory size is $O(|E| n)$. We have attributed a higher time to [52] in the table, since we wanted to remove its assumption of a known leader, to make a fair comparison to the later papers who do not rely on this assumption.

To remove the assumption, in the first entry we assumed the usage of the only leader election known at the time of [52]. That is, in [3], the first self-stabilizing leader election algorithm was proposed in order to remove the assumptions of [52] that a leader and a spanning tree are given. The combination of [3] and [52] implied a self-stabilizing MST in $O\left(n^{2}\right)$ time. (Independently, a leader election algorithm was also presented by [6]; however, we cannot use it here since it needed an extra assumption that a bound on $n$ was known; also, its higher time complexity would have driven the complexity of the transformed MST algorithm higher than the $O\left(n^{2}\right)$ stated above.)

Using unbounded space, the time of self-stabilizing leader election was later improved even to $O(D)$ (the actual diameter) [4, 27]. The bounded memory algorithms of [10] or [1, 28], together with [52] and [9], yield a self-stabilizing MST algorithm using $O(n|E| \log n)$ bits per node and time $O(D \log n)$ or $O(n)$.

Antonoiu and Srimani [5] presented a self stabilizing algorithm whose complexities were not analyzed. As mentioned by [48], the model in that paper can be transformed to the models of the other papers surveyed here, at a high translation costs. Hence, the complexities of the algorithm of [5] may grow even further when stated in these models. Gupta and Srimani [47] presented an $O(n \log n)$ bits algorithm. Higham and Liang [48] improved the core memory requirement to $O(\log n)$, however, the time complexity went up again to $\Omega(n|E|)$. An algorithm with a similar time complexity and a similar memory per node was also presented by Blin, PotopButucaru, Rovedakis, and Tixeuil [18]. This latter algorithm exchanges less bits with neighbours than does the algorithm of [48]. The algorithm of [18] addressed also another goal- even during stabilization it is loop free. That is, it also maintains a tree at all times (after reaching an initial tree). This algorithm assumes the existence of a unique leader in the network (while the algorithm in the current paper does not). However, this does not seem to affect the order of magnitude of the time complexity.

Note that the memory size in the last two algorithms above is the same as in the current paper. However, their time complexity is $O(|E| n)$ versus $O(n)$ in the current paper. The time complexity of the algorithm of Blin, Dolev, Potop-Butucaru, and Rovedakis [17] improved the time complexity of [18, 48] to $O\left(n^{2}\right)$ but at the cost of growing the memory usage to $O\left(\log ^{2} n\right)$. This may be the first paper using labeling schemes for the design of a self-stabilizing MST protocol, as well as the first paper implementing the algorithm by Gallager, Humblet, and Spira in a self-stabilizing manner without using a general transformer.

Additional studies about MST verification in various models appeared in [26, 30, 31, 53, 54, 55]. In particular, Kor et al. [53] shows that the verification from scratch (without labels) of an MST requires $\Omega(\sqrt{n}+D)$ time and $\Omega(|E|)$ messages, and that these bounds are tight up to poly-logarithmic factors. We note that the memory complexity was not considered in [53], and indeed the memory used therein is much higher than the one used in the current paper. The time lower bound proof in [53] was later extended in [26] to apply for a variety of verification and computation tasks.

This paper has results concerning distributed verification. Various additional papers dealing with verification have appeared recently, the models of some of them are rather different than the model here. Verification in the $\mathcal{L O C} \mathcal{A} \mathcal{L}$ model (where congestion is abstracted away) was studied in [37] from a computational complexity perspective. That paper presents various complexity classes, shows separation between them, and provides complete problems for these classes. In particular, the class NLD defined therein exhibits similarities to the notion of proof labeling schemes. Perhaps the main result in [37] is a sharp threshold for the impact of randomization on local 
Table 1: Comparing self-stabilizing MST construction algorithms

\begin{tabular}{|c|c|c|c|c|}
\hline Algorithm & space & time & Asynch & comment \\
\hline$[52]+[3]+[9]$ & $O(|E| n)$ & $O\left(n^{2}\right)$ & yes & \\
\hline$[52]+[4]+[9]$ & unbounded & $O(D)$ & yes & $\begin{array}{l}\text { The } 2 \text { nd component can be re- } \\
\text { placed by [27], assuming the } \\
\mathcal{L} \mathcal{O C} \mathcal{A} \mathcal{L} \text { model. }\end{array}$ \\
\hline$[52]+9]+10$ & $O(|E| n) \log n$ & $O(\min \{D \log n, n\})$ & yes & $\begin{array}{l}\text { The third component here can be } \\
\text { replaced by [1] or by [28. }\end{array}$ \\
\hline 47 & $O(n \log n)$ & $O(n)$ & no & $\begin{array}{l}\text { Implies an } O\left(n^{2}\right) \text { time bound in } \\
\text { asynchronous networks, assum- } \\
\text { ing a good bound on the net- } \\
\text { work size is known. The time is } \\
\text { based on assuming the } \mathcal{L O C \mathcal { A }} \\
\text { model. }\end{array}$ \\
\hline 48 & $O(\log n)$ & $\Omega(|E| n)$ & yes & $\begin{array}{l}\text { The time complexity is based } \\
\text { on the assumption that a good } \\
\text { bound on the network diameter } \\
\text { is known. }\end{array}$ \\
\hline 18 & $O(\log n)$ & $\Omega(|E| n)$ & yes & $\begin{array}{l}\text { Aims to exchanging less bits } \\
\text { with neighbours than [48]. As- } \\
\text { sumes a leader is known. }\end{array}$ \\
\hline 17 & $O\left(\log ^{2}(n)\right)$ & $O\left(n^{2}\right)$ & yes & \\
\hline Current paper & $O(\log n)$ & $O(n)$ & yes & \\
\hline
\end{tabular}


decision of hereditary languages. Following that paper, [38] showed that the threshold in [37] holds also for any non-hereditary language, assuming it is defined on path topologies. In addition, [38] showed further limitations of randomness, by presenting a hierarchy of languages, ranging from deterministic, on the one side of the spectrum, to almost complete randomness, on the other side. Still, in the $\mathcal{L O C} \mathcal{A} \mathcal{L}$ model, [39] studied the impact of assuming unique identifiers on local decision. We stress that the memory complexity was not considered in neither [37] nor in its follow-up papers [38, 39].

\subsection{Our results}

This paper contains the following two main results.

(1) Solving an open problem posed by Awerbuch and Varghese [15]: In the context of self-stabilization, an open problem posed in [15] is to find a (synchronous) MST verification algorithm whose time complexity is smaller than the MST computation time, yet with a small memory. Our first main result solves this question positively by constructing a time efficient self-stabilizing verification algorithm for an MST while using optimal memory size, that is $O(\log n)$ bits of memory per node. More specifically, the verification scheme takes as input a distributed structure claimed to be an MST. If the distributed structure is indeed an MST, and if a marker algorithm properly marked the nodes to allow the verification, and if no faults occur, then our algorithm outputs accept continuously in every node. However, if faults occur (including the case that the structure is not, in fact, an MST, or that the marker did not perform correctly), then our algorithm outputs reject in at least one node. This reject is outputted in time $O\left(\log ^{2} n\right)$ after the faults cease, in a synchronous network. (Recall, lower bounds which are polynomial in $n$ for MST construction are known even for synchronous networks [58, 62].) In asynchronous networks, the time complexity of our verification scheme grows to $O\left(\Delta \log ^{3} n\right)$. We also show that $\Omega(\log n)$ time is necessary if the memory size is restricted to $O(\log n)$, even in synchronous networks. Another property of our verification scheme is that if $f$ faults occurred, then, within the required detection time above, they are detected by some node in the $O(f \log n)$ locality of each of the faults. Moreover, we present a distributed implementation of the marker algorithm whose time complexity for assigning the labels is $O(n)$, under the same memory size constraint of $O(\log n)$ memory bits per node.

(2) Constructing an asynchronous self-stabilizing MST construction algorithm which uses optimal memory $(O(\log n)$ bits) and runs in $O(n)$ time: In our second main result, we show how to enhance a known transformer that makes input/output algorithms self-stabilizing. It now takes as input an efficient construction algorithm and an efficient self-stabilizing proof labeling scheme, and produces an efficient self-stabilizing algorithm. When used with our verification scheme, the transformer produces a memory optimal self-stabilizing MST construction algorithm, whose time complexity, namely, $O(n)$, is significantly better even than that of previous algorithms. (Recall, the time complexity of previous MST algorithms that used $\Omega\left(\log ^{2} n\right)$ memory bits per node was $O\left(n^{2}\right)$, and the time for optimal space algorithms was $O(n|E|)$.) Inherited from our verification scheme, our self-stabilising MST construction algorithm also has the following two properties. First, if faults occur after the construction ended, then they are detected by some nodes within $O\left(\log ^{2} n\right)$ time in synchronous networks, or within $O\left(\Delta \log ^{3} n\right)$ time in asynchronous ones, and second, if $f$ faults occurred, then, within the required detection time above, they are detected within the $O(f \log n)$ locality of each of the faults. We also show how to improve these two properties, at the expense of some increase in the memory. 


\subsection{Outline}

Preliminaries and some examples of simple, yet useful, proof labeling schemes are given in Section 2 . An intuition is given in Section 3 A building block is then given in Section 4. Namely, that section describes a synchronous MST construction algorithm in $O(\log n)$ bits memory size and $O(n)$ time. Section 5 describes the construction of parts of the labeling scheme. Those are the parts that use labeling schemes of the kind described in [55]- namely, schemes that can be verified in one time unit. These parts use the MST construction (of Section 47) to assign the labels. Sections 6, 7, and 8 describe the remaining part of the labeling scheme. This part is a labeling scheme by itself, but of a new kind. It saves on memory by distributing information. Specifically, Section 6 describes how the labels should look if they are constructed correctly (and if an MST is indeed represented in the graph). The verifications, in the special case that no further faults occur, is described in Section 77. This module verifies (alas, not in constant time) by moving the distributed information around, for a "distributed viewing". Because the verification is not done in one time unit, it needs to be made self stabilizing. This is done in Section 8 . Section 9 presents a lower bound for the time of a proof labeling scheme for MST that uses only logarithmic memory. (Essentially, the proof behind this lower bound is based on a simple reduction, using the rather complex lower bound given in [54].)

The efficient self-stabilizing MST algorithm is given in Section 10. Using known transformers, we combine efficient MST verification schemes and (non-self-stabilizing) MST construction schemes to yield efficient selfstabilizing schemes. The MST construction algorithm described in Section 4 is a variant of some known time efficient MST construction algorithms. We show there how those can also be made memory efficient (at the time, this complexity measure was not considered), and hence can be used as modules for our optimal memory self-stabilizing MST algorithm.

\section{Preliminaries}

\subsection{Some general definitions}

We use rather standard definitions; a reader unfamiliar with these notions may refer to the model descriptions in the rich literature on these subjects. In particular, we use rather standard definitions of self-stabilization (see, e.g. [32]). Note that the assumptions we make below on time and time complexity imply (in self stabilization jargon) a distributed daemon with a very strong fairness. When we speak of asynchronous networks, this implies a rather fine granularity of atomicity. Note that the common self stabilization definitions include the definitions of faults. We also use standard definitions of graph theory (including an edge weighted graph $G=(V, E)$, with weights that are polynomial in $n=|V|$ ) to represent a network (see, e.g. [35]). Each node $v$ has a unique identity $\operatorname{ID}(v)$ encoded using $O(\log n)$ bits. For convenience, we assume that each adjacent edge of each node $v$ has some label that is unique at $v$ (edges at different nodes may have the same labels). This label, called a port-number, is known only to $v$ and is independent of the port-number of the same edge at the other endpoint of the edge. (Clearly, each port-number can be assumed to be encoded using $O(\log n)$ bits). Moreover, the network can store an object such as an MST (Minimum Spanning Tree) by having each node store its component of the representation. A component $c(v)$ at a node $v$ includes a collection of pointers (or port-numbers) to neighbours of $v$, and the collection of the components of all nodes induces a subgraph $H(G)$ (an edge is included in $H(G)$ if and only if at least one of its end-nodes points at the other end-node). In the verification scheme considered in this current paper, $H(G)$ is supposed to be an MST and for simplicity, we assume that the component of each node contains a single pointer (to the parent, if that node is not defined as the root). It is not difficult to extend our verification scheme to hold also for the case where each component can contain several pointers. Note that the definitions in this paragraph imply a lower bound of $\Omega(\log n)$ bits on the memory required at each node to 
even represent an MST (in graphs with nodes of high degree).

Some additional standard ([40]) parts of the model include the assumption that the edge weights are distinct. As noted often, having distinct edge weights simplifies our arguments since it guarantees the uniqueness of the MST. Yet, this assumption is not essential. Alternatively, in case the graph is not guaranteed to have distinct edge weights, we may modify the weights locally as was done in [53]. The resulted modified weight function $\omega^{\prime}(e)$ not only assigns distinct edge weights, but also satisfies the property that the given subgraph $H(G)$ is an MST of $G$ under $\omega(\cdot)$ if and only if $H(G)$ is an MST of $G$ under $\omega^{\prime}(\cdot) !$

We use the (rather common) ideal time complexity which assumes that a node reads all of its neighbours in at most one time unit, see e.g. [18, 17]. Our results translate easily to an alternative, stricter, contention time complexity, where a node can access only one neighbour in one time unit. The time cost of such a translation is at most a multiplicative factor of $\Delta$, the maximum degree of a node (it is not assumed that $\Delta$ is known to nodes).

As is commonly assumed in the case of self-stabilization, each node has only some bounded number of memory bits available to be used. Here, this amount of memory is $O(\log n)$.

\subsection{Using protocols designed for message passing}

We use a self stabilizing transformer of Awerbuch and Varghese as a building block [15]. That protocol was designed for the message passing model. Rather than modifying that transformer to work on the model used here (which would be very easy, but would take space), we use emulation. That is, we claim that any self stabilizing protocol designed for the model of [15] (including the above transformer) can be performed in the model used here, adapted from [18, 17]. This is easy to show: simply use the current model to implement the links of the model of [15]. To send a message from node $v$ to its neighbour $u$, have $v$ write its shared variable that (only $v$ and) $u$ can read. This value can be read by $u$ after one time unit in a synchronous network as required from a message arrival in the model of [15]. Hence, this is enough for synchronous networks.

In an asynchronous network, we need to work harder to simulate the sending and the receiving of a message, but only slightly harder, given known art. Specifically, in an asynchronous network, an event occurs at $u$ when this message arrives. Without some additional precaution on our side, $u$ could have read this value many times (per one writing) resulting in duplications: multiple message "arriving" while we want to emulate just one message. This is solved by a self stabilizing data link protocol, such as the one used by [3], since this is also the case in a data link protocol in message passing systems where a link may loose a package. There, a message is sent repeatedly, possibly many times, until an acknowledgement from the receiver tells the sender that the message

\footnotetext{
${ }^{1}$ We note, the standard technique (e.g., [40]) for obtaining unique weights is not sufficient for our purposes. Indeed, that technique orders edge weights lexicographically: first, by their original weight $\omega(e)$, and then, by the identifiers of the edge endpoints. This yields a modified graph with unique edge weights, and an MST of the modified graph is necessarily an MST of the original graph. For construction purposes it is therefore sufficient to consider only the modified graph. Yet, this is not the case for verification purposes, as the given subgraph can be an MST of the original graph but not necessarily an MST of the modified graph. While the authors in [53] could not guarantee that any MST of the original graph is an MST of the modified graph (having unique edge weights), they instead make sure that the particular given subgraph $T$ is an MST of the original graph if and only if it is an MST of modified one. This condition is sufficient for verification purposes, and allows one to consider only the modified graph. For completeness, we describe the weight-modification in [53]. To obtain the modified graph, the authors in [53] employ the technique, where edge weights are lexicographically ordered as follows. For an edge $e=(v, u)$ connecting $v$ to its neighbour $u$, consider first its original weight $\omega(e)$, next, the value $1-Y_{u}^{v}$ where $Y_{u}^{v}$ is the indicator variable of the edge $e$ (indicating whether $e$ belongs to the candidate MST to be verified), and finally, the identifiers of the edge endpoints, $\operatorname{ID}(v)$ and $\operatorname{ID}(u)$ (say, first comparing the smaller of the two identifiers of the endpoints, and then the larger one). Formally, let $\omega^{\prime}(e)=\left\langle\omega(e), 1-Y_{u}^{v}, \operatorname{ID}_{m i n}(e), \operatorname{ID}_{m a x}(e)\right\rangle$, where $\operatorname{ID}_{m i n}(e)=\min \{\operatorname{ID}(v), \operatorname{ID}(u)\}$ and $\operatorname{ID}_{\max }(e)=\max \{\operatorname{ID}(v), \operatorname{ID}(u)\}$. Under this weight function $\omega^{\prime}(e)$, edges with indicator variable set to 1 will have lighter weight than edges with the same weight under $\omega(e)$ but with indicator variable set to 0 (i.e., for edges $e_{1} \in T$ and $e_{2} \notin T$ such that $\omega\left(e_{1}\right)=\omega\left(e_{2}\right)$, we have $\omega^{\prime}\left(e_{1}\right)<\omega^{\prime}\left(e_{2}\right)$ ). It follows that the given subgraph $T$ is an MST of $G$ under $\omega(\cdot)$ if and only if $T$ is an MST of $G$ under $\omega^{\prime}(\cdot)$. Moreover, since $\omega^{\prime}(\cdot)$ takes into account the unique vertex identifiers, it assigns distinct edge weights.
} 
arrived. The data link protocol overcomes the danger of duplications by simply numbering the messages modulo some small number. That is, the first message is sent repeatedly with an attached "sequence number" zero, until the first acknowledgement arrives. All the repetitions of the second message have as attachments the sequence number 1, etc. The receiver then takes just one of the copies of the first message, one of the copies of the second, etc. A self stabilized implementation of this idea in a shared memory model appears in [3] using (basically, to play the role of the sequence number) an additional shared variable called the "toggle", which can take one of three values ${ }^{2}$ When $u$ reads that the toggle of $v$ changes, $u$ can emulate the arrival of a message. In terms of time complexity, this protocol takes a constant time, and hence sending (an emulated) message still takes a constant time (in terms of complexity only) as required to emulate the notion of ideal time complexity of [18, 17]. Note that the memory is not increased.

\subsection{Wave\&Echo}

We use the well known Wave\&Echo (PIF) tool. For details, the readers are referred to [21, 63]. For completeness, we remind the reader of the overview of Wave\&Echo when performed over a rooted tree. It is started by the tree root, and every node who receives the wave message forwards it to its children. The wave can carry a command for the nodes. A leaf receiving the wave, computes the command, and sends the output to its parent. This is called an echo. A parent, all of whose children echoed, computes the command itself (possibly using the outputs sent by the children) and then sends the echo (with its own output) to its parent. The Wave\&Echo terminates at the root when all the children of the root echoed, and when the root executed the command too.

In this paper, the Wave\&Echo activations carry various commands. Let us describe first two of these commands, so that they will also help clarify the notion of Wave\&Echo and its application. The first example is the command to sum up values residing at the nodes. The echo of a leaf includes its value. The echo of a parent includes the sum of its own value and the sums sent by its children. Another example is the counting of the nodes. This is the same as the sum operation above, except that the initial value at a node is 1 . Similarly to summing up, the operation performed by the wave can also be a logical OR.

\subsection{Proof labeling schemes}

In [54, 55, 46], the authors consider a framework for maintaining a distributed proof that the network satisfies some given predicate $\Psi$, e.g., that $H(G)$ is an MST. We are given a predicate $\Psi$ and a graph family $\mathcal{F}$ (in this paper, if $\Psi$ and $\mathcal{F}$ are omitted, then $\Psi$ is MST and $\mathcal{F}$ (or $\mathcal{F}(n)$ ) is all connected undirected weighted graphs with $n$ nodes). A proof labeling scheme (also referred to as a verification algorithm) includes the following two components.

- A marker algorithm $\mathcal{M}$ that generates a label $\mathcal{M}(v)$ for every node $v$ in every graph $G \in \mathcal{F}$.

- A verifier, that is a distributed algorithm $\mathcal{V}$, initiated at each node of a labeled graph $G \in \mathcal{F}$, i.e., a graph whose nodes $v$ have labels $L(v)$ (not necessarily correct labels assigned by a marker). The verifier at each node is initiated separately, and at an arbitrary time, and runs forever. The verifier may raise an alarm at some node $v$ by outputting "no" at $v$.

Intuitively, if the verifier at $v$ raises an alarm, then it detected a fault. That is, for any graph $G \in \mathcal{F}$,

\footnotetext{
${ }^{2}$ That protocol, called "the strict discipline" in [3], actually provides a stronger property (emulating a coarser grained atomicity), not used here.
} 
- If $G$ satisfies the predicate $\Psi$ and if the label at each node $v$ is $\mathcal{M}(v)$ (i.e., the label assigned to $v$ by the marker algorithm $\mathcal{M}$ ) then no node raises an alarm. In this case, we say that the verifier accepts the labels.

- If $G$ does not satisfy the predicate $\Psi$, then for any assignment of labels to the nodes of $G$, after some finite time $t$, there exists a node $v$ that raises an alarm. In this case, we say that the verifier rejects the labels.

Note that the first property above concerns only the labels produced by the marker algorithm $\mathcal{M}$, while the second must hold even if the labels are assigned by some adversary. We evaluate a proof labeling scheme $(\mathcal{M}, \mathcal{V})$ by the following complexity measures.

- The memory size: the maximum number of bits stored in the memory of a single node $v$, taken over all the nodes $v$ in all graphs $G \in \mathcal{F}(n)$ that satisfy the predicate $\Psi$ (and over all the executions); this includes: (1) the bits used for encoding the identity $\operatorname{ID}(v),(2)$ the marker memory: number of bits used for constructing and encoding the labels, and (3) the verifier memory: the number of bits used during the operation of the verifiet ${ }^{3}$.

- The (ideal) detection time: the maximum, taken over all the graphs $G \in \mathcal{F}(n)$ that do not satisfy the predicate $\Psi$ and over all the labels given to nodes of $G$ by adversaries (and over all the executions), of the time $t$ required for some node to raise an alarm. (The time is counted from the starting time, when the verifier has been initiated at all the nodes.)

- The detection distance: for a faulty node $v$, this is the (hop) distance to the closest node $u$ raising an alarm within the detection time after the fault occurs. The detection distance of the scheme is the maximum, taken over all the graphs having at most $f$ faults, and over all the faulty nodes $v$ (and over all the executions), of the detection distance of $v$.

- The (ideal) construction-time: the maximum, taken over all the graphs $G \in \mathcal{F}(n)$ that satisfy the predicate $\Psi$ (and over all the executions), of the time required for the marker $\mathcal{M}$ to assign labels to all nodes of $G$. Unless mentioned otherwise, we measure construction-time in synchronous networks only.

In our terms, the definitions of [54, 55] allowed only detection time complexity 1 . Because of that, the verifier of [54, 55] at a node $v$, could only consult the neighbours of $v$. Whenever we use such a scheme, we refer to it as a 1-proof labeling scheme, to emphasis its running time. Note that a 1-proof labelling scheme is trivially self-stabilzying. (In some sense, this is because they "silently stabilize" [33], and "snap stabilize" [20].) Also, in [54, 55], if $f$ faults occurred, then the detection distance was $f$.

\subsection{Generalizing the complexities to a computation}

In Section 2.4, we defined the memory size, detection time and the detection distance complexities of a verification algorithm. When considering a (self-stabilizing) computation algorithm, we extend the notion of the memory size to include also the bits needed for encoding the component $c(v)$ at each node. Recall, the definition of a component $c(v)$ in general, and the special case of $c(v)$ for MST, are given in Section2. (Recall, from Section 2.4, that the size of the component was excluded from the definition of memory size for verification because, there, the designer of the verification scheme has no control over the nodes' components.)

\footnotetext{
${ }^{3}$ Note that we do not include the number of bits needed for storing the component $c(v)$ at each node $v$. Recall that for simplicity, we assume here that each component contains a single pointer, and therefore, the size of each component is $O(\log n)$ bits. Hence, for our purposes, including the size of a component in the memory complexity would not increase the asymptotical size of the memory, anyways. However, in the general case, if multiple pointers can be included in a component, then the number of bits needed for encoding a component would potentially be as large as $O(\Delta \log n)$. Since, in this case, the verification scheme has no control over the size of the component, we decided to exclude this part from the definition of the memory complexity.
} 
The notions of detection time and the detection distance can be carried to the very common class of selfstabilizing computation algorithms that use fault detection. (Examples for such algorithms are algorithms that have silent stabilization [33].) Informally, algorithms in this class first compute an output. After that, all the nodes are required to stay in some output state where they (1) output the computation result forever (unless a fault occurs); and (2) check repeatedly until they discover a fault. In such a case, they recompute and enter an output state again. Let us term such algorithms detection based self-stabilizing algorithms. We define the detection time for such algorithms to be the time from a fault until the detection. However, we only define the detection time (and the detection distance) for faults that occur after all the nodes are in their output states. (Intuitively, in the other cases, stabilization has not been reached yet anyhow.) The detection distance is the distance from a node where a fault occurred to the closest node that detected a fault.

\subsection{Some examples of 1-proof labeling schemes}

As a warm-up exercise, let us begin by describing several simple 1-proof labeling schemes, which will be useful later in this paper. The first two examples are taken from [55] and are explained there in more details. The reader familiar with proof labeling schemes may skip this subsection.

- Example 1: A spanning tree. (Example SP) Let $f_{\text {span }}$ denote the predicate such that for any input graph $G$ satisfies, $f_{\text {span }}(G)=1$ if $H(G)$ is a spanning tree of $G$, and $f_{\text {span }}(G)=0$ otherwise. We now describe an efficient 1-proof labeling scheme $(\mathcal{M}, \mathcal{V})$ for the predicate $f_{\text {span }}$ and the family of all graphs. Let us first describe the marker $\mathcal{M}$ operating on a "correct instance", i.e., a graph $G$ where $T=H(G)$ is indeed a spanning tree of $G$. If there exists a node $u$ whose component does not encode a pointer to any of its adjacent edges (observe that there can be at most one such node), we root $T$ at $u$. Otherwise, there must be two nodes $v$ and $w$ whose components point at each other. In this case, we root $T$ arbitrarily at either $v$ or $w$. Note that after rooting $T$, the component at each non-root node $v$ points at $v$ 's parent. The label given by $\mathcal{M}$ to a node $v$ is composed of two parts. The first part encodes the identity $\operatorname{ID}(r)$ of the root $r$ of $T$, and the second part of the label of $v$ encodes the distance (assuming all weights of edges are 1) between $v$ and $r$ in $T$.

Given a labeled graph, the verifier $\mathcal{V}$ operates at a node $v$ as follows: first, it checks that the first part of the label of $v$ agrees with the first part of the labels of $v$ 's neighbours, i.e., that $v$ agrees with its neighbours on the identity of the root. Second, let $d(v)$ denote the number encoded in the second part of $v$ 's label. If $d(v)=0$ then $\mathcal{V}$ verifies that $\operatorname{ID}(v)=\operatorname{ID}(r)$ (recall that $\operatorname{ID}(r)$ is encoded in the first part of $v$ 's label). Otherwise, if $d(v) \neq 0$ then the verifier checks that $d(v)=d(u)+1$, where $u$ is the node pointed at by the component at $v$. If at least one of these conditions fails, the verifier $\mathcal{V}$ raises an alarm at $v$. It is easy to get convinced that $(\mathcal{M}, \mathcal{V})$ is indeed a 1-proof labeling scheme for the predicate $f_{\text {span }}$ with memory size $O(\log n)$ and construction time $O(n)$.

Remark. Observe that in case $T=H(G)$ is indeed a (rooted) spanning tree of $G$, we can easily let each node $v$ know which of its neighbours in $G$ are its children in $T$ and which is its parent. Moreover, this can be done using one unit of time and label size $O(\log n)$ bits. To see this, for each node $v$, we simply add to its label its identity $\operatorname{ID}(v)$ and the identity $\operatorname{ID}(u)$ of its parent $u$. The verifier at $v$ first verifies that $\operatorname{ID}(v)$ is indeed encoded in the right place of its label. It then looks at the label of its parent $u$, and checks that $v$ 's candidate for being the identity of $u$ is indeed $\operatorname{ID}(u)$. Assume now that these two conditions are satisfied at each node. Then, to identify a child $w$ in $T$, node $v$ should only look at the labels of its neighbours in $G$ and see which of them encoded $\operatorname{ID}(v)$ in the designated place of its label.

- Example 2: Knowing the number of nodes (Example NumK) Denote by $f_{\text {size }}$ the boolean predicate such that $f_{\text {size }}(G)=1$ if and only if one designated part of the label $L(v)$ at each node $v$ encodes the number of nodes in 
$G$ (informally, when $f_{\text {size }}$ is satisfied, we say that each node 'knows' the number of nodes in $G$ ). Let us denote the part of the label of $v$ that is supposed to hold this number by $L^{\prime}(v)$.

In [55], the authors give a 1-proof labeling scheme $(\mathcal{M}, \mathcal{V})$ for $f_{\text {size }}$ with memory size $O(\log n)$. The idea behind their scheme is simple. First, the verifier checks that $L^{\prime}(u)=L^{\prime}(v)$ for every two adjacent nodes $u$ and $v$ (if this holds at each node then all nodes must hold the same candidate for being the number of nodes). Second, the marker constructs a spanning tree $T$ rooted at some node $r$ (and verifies that this is indeed a spanning tree using the Example SP above). Third, the number of nodes in $T$ is aggregated upwards along $T$ towards its root $r$, by keeping at the label $\mathcal{M}(v)$ of each node $v$, the number of nodes $n(v)$ in the subtree of $T$ hanging down from $v$. This again is easily verified by checking at each node $v$ that $n(v)=1+\sum_{u \in \operatorname{Child}(v)} n(u)$, where $\operatorname{Child}(v)$ is the set of children of $v$. Finally, the root verifies that $n(r)=L^{\prime}(r)$. It is straightforward that $(\mathcal{M}, \mathcal{V})$ is indeed a 1-proof labeling scheme for the predicate $f_{\text {size }}$ with memory size $O(\log n)$ and construction time $O(n)$.

$\circ$ Example 3: An upper bound on the diameter of a tree (Example ED IAM) Consider a tree $T$ rooted at $r$, and let $h$ denote the height of $T$. Denote by $f_{\text {height }}$ the boolean predicate such that $f_{\text {height }}(T)=1$ if and only if one designated part of the label $L(v)$ at each node encodes the same value $x$, where $h \leq x$ (informally, when $f_{\text {height }}$ is satisfied, we say that each node 'knows' an upper bound of $2 x$ on the diameter $D$ of $T$ ). Let us denote the part of the label of $v$ that is supposed to hold this number by $L^{\prime}(v)$. We sketch a simple 1-proof labeling scheme $(\mathcal{M}, \mathcal{V})$ for $f_{\text {height }}$. First, the verifier checks that $L^{\prime}(u)=L^{\prime}(v)$ for every two adjacent nodes $u$ and $v$ (if this holds at each node then all nodes must hold the same value $x$ ). Second, similarly to the proof labeling scheme for $f_{\text {span }}$ given in Example SP above, the label in each node $v$ contains the distance $d(v)$ in the tree from $v$ to the root. Each non-root node verifies that the distance written at its parent is one less than the distance written at itself, and the root verifies that the distance written at itself is 0 . Finally, each node $v$ verifies also that $x \geq d(v)$. If no node raises an alarm then $x$ is an upper bound on the height. On the other hand, if the value $x$ is the same at all nodes and $x$ is an upper bound on the height then no node raises an alarm. Hence the scheme is a 1-proof labeling scheme for the predicate $f_{\text {height }}$ with memory size $O(\log n)$ and construction time $O(n)$.

\section{Overview of the MST verification scheme and the intuition behind it}

The final MST construction algorithm makes use of several modules. The main technical contribution of this paper is the module that verifies that the collection of nodes' components is indeed an MST. This module in itself is composed of multiple modules. Some of those, we think may be useful by themselves. To help the reader avoid getting lost in the descriptions of all the various modules, we present, in this section, an overview of the MST verification part.

Given a graph $G$ and a subgraph that is represented distributively at the nodes of $G$, we wish to produce a self-stabilizing proof labeling scheme that verifies whether the subgraph is an MST of $G$. By first employing the (self-stabilizing) 1-proof labeling scheme mentioned in Example SP, we may assume that the subgraph is a rooted spanning tree of $G$ (otherwise, at least one node would raise an alarm). Hence, from now on, we focus on a spanning tree $T=(V(G), E(T))$ of a weighted graph $G=(V(G), E(G))$, rooted at some node $r(T)$, and aim at verifying the minimality of $T$.

\subsection{Background and difficulties}

From a high-level perspective, the proof labeling scheme proves that $T$ could have been computed by an algorithm that is similar to that of GHS, the algorithm of Gallager, Humblet, and Spira's described in [40]. This leads to a simple idea: when $T$ is a tree computed by such an algorithm, $T$ is an MST. Let us first recall a few terms from 
[40]. A fragment $F$ denotes a connected subgraph of $T$ (we simply refer it to a subtree). Given a fragment $F$, an edge $(v, u) \in E(G)$ whose one endpoint $v$ is in $F$, while the other endpoint $u$ is not, is called outgoing from $F$. Such an edge of minimum weight is called a minimum outgoing edge from $F$. A fragment containing a single node is called a singleton. Recall that GHS starts when each node is a fragment by itself. Essentially, fragments merge over their minimum outgoing edges to form larger fragments. That is, each node belongs to one fragment $F_{1}$, then to a larger fragment $F_{2}$ that contains $F_{1}$, etc. This is repeated until one fragment spans the network. A tree constructed in that way is an MST. Note that in GHS, the collection of fragments is a laminar family, that is, for any two fragments $F$ and $F^{\prime}$ in the collection, if $F \cap F^{\prime} \neq \emptyset$ then either $F \subseteq F^{\prime}$ or $F^{\prime} \subseteq F$ (see, e.g. [44]). Moreover, each fragment has a level; in the case of $v$ above, $F_{2}$ 's level is higher than that of $F_{1}$. This organizes the fragments in a hierarchy $\mathcal{H}$, which is a tree whose nodes are fragments, where fragment $F_{1}$ is a descendant in $\mathcal{H}$ of $F_{2}$ if $F_{2}$ contains $F_{1}$. GHS manages to ensure that each node belongs to at most one fragment at each level, and that the total number of levels is $O(\log n)$. Hence, the hierarchy $\mathcal{H}$ has $O(\log n)$ height.

The marker algorithm in our proof labeling scheme performs, in some sense, a reverse operation. If $T$ is an MST, the marker "slices" it back into fragments. Then, the proof labeling scheme computes for each node $v$ :

- The (unique) name of each of the fragments $F_{j}$ that $v$ belongs to,

- the level of $F_{j}$, and

- the weight of $F_{j}$ 's minimum outgoing edge.

Note that each node participates in $O(\log n)$ fragments, and the above "piece of information" per fragment requires $O(\log n)$ bits. Hence, this is really too much information to store in one node. As we shall see later, the verification scheme distributes this information and then brings it to the node without violating the memory size bound $O(\log n)$. For now, it suffices to know that given these pieces of information and the corresponding pieces of information of their neighbours, the nodes can verify that $T$ could have been constructed by an algorithm similar to GHS. That way, they verify that $T$ is an MST. Indeed, the 1-proof labeling schemes for MST verification given in [54, 55] follow this idea employing memory size of $O\left(\log ^{2} n\right)$ bits. (There, each node keeps $O(\log n)$ pieces, each of $O(\log n)$ bits.)

The current paper allows each node to hold only $O(\log n)$ memory bits. Hence, a node has room for only a constant number of such pieces of information at a time. One immediate idea is to store some of $v$ 's pieces in some other nodes. Whenever $v$ needs a piece, some algorithm should move it towards $v$. Moving pieces would cost time, hence, realizing some time versus memory size trade-off.

Unfortunately, the total (over all the nodes) number of pieces in the schemes of [54, 55] is $\Omega(n \log n)$. Any way one would assign these pieces to nodes would result in the memory of some single node needing to store $\Omega(\log n)$ pieces, and hence, $\Omega\left(\log ^{2} n\right)$ bits. Thus, one technical step we used here is to reduce the total number of pieces to $O(n)$, so that we could store at each node just a constant number of such pieces. However, each node still needs to use $\Omega(\log n)$ pieces. That is, some pieces may be required by many nodes. Thus, we needed to solve also a combinatorial problem: locate each piece "close" to each of the nodes needing it, while storing only a constant number of pieces per node.

The solution of this combinatorial problem would have sufficed to construct the desired scheme in the $\mathcal{L O C} \mathcal{A} \mathcal{L}$ model [61]. There, node $v$ can "see" the storage of nearby nodes. However, in the congestion aware model, one actually needs to move pieces from node to node, while not violating the $O(\log n)$ memory per node constraint. This is difficult, since, at the same time $v$ needs to see its own pieces, other nodes need to see their own ones. 


\subsection{A very high level overview}

Going back to GHS, one may notice that its correctness follows from the combination of two properties:

- P1. (Well-Forming) The existence of a hierarchy tree $\mathcal{H}$ of fragments, satisfying the following:

- Each fragment $F \in \mathcal{H}$ has a unique selected outgoing edge (except when $F$ is the whole tree $T$ ).

- A (non-singleton) fragment is obtained by merging its children fragments in $\mathcal{H}$ through their selected outgoing edges.

- P2. (Minimality) The selected outgoing edge of each fragment is its minimal outgoing edge.

In our proof labeling scheme, we verify the aforementioned two properties separately. In Section 5 , we show how to verify the first property, namely, property Well-Forming. This turns out to be a much easier task than verifying the second property. Indeed, the Well-Forming property can be verified using a 1-proof labeling scheme, while still obeying the $O(\log n)$ bits per node memory constraint. Moreover, the techniques we use for verifying the Well-Forming are rather similar to the ones described in [55]. The more difficult verification task, namely, verifying the Minimality property $\mathrm{P} 2$, is described in Section 6. This verification scheme requires us to come up with several new techniques which may be considered as contributions by themselves. We now describe the intuition behind these verifications.

\subsection{Verifying the Well-Forming property (described in detail in Sections 4 and 5 )}

We want to show that $T$ could have been produced by an algorithm similar to GHS. Crucially, since we care about the memory size, we had to come up with a new MST construction algorithm that is similar to GHS but uses only $O(\log n)$ memory bits per node and runs in time $O(n)$. This MST construction algorithm, called SYNC_MST, can be considered as a synchronous variant of GHS and is described in Section 4 .

Intuitively, for a correct instance (the case $T$ is an MST), the marker algorithm $\mathcal{M}$ produces a hierarchy of fragments $\mathcal{H}$ by following the new MST construction algorithm described in Section 4 . Let $\ell=O(\log n)$ be the height of $\mathcal{H}$. For a fixed level $j \in[0, \ell]$, it is easy to represent the partition of the tree into fragments of level $j$ using just one bit per node. That is, the root $r^{\prime}$ of each fragment $F^{\prime}$ of level $j$ has 1 in this bit, while the nodes in $F^{\prime} \backslash\left\{r^{\prime}\right\}$ have 0 in this bit. Note, these nodes in $F^{\prime} \backslash\left\{r^{\prime}\right\}$ are the nodes below $r^{\prime}$ (further away from the root of $T$ ), until (and not including) reaching additional nodes whose corresponding bit is 1 . Hence, to represent the whole hierarchy, it is enough to attach a string of length $\ell+1$-bits at each node $v$. The string at a node $v$ indicates, for each level $j \in[0, \ell]$, whether $v$ is the root of the fragment of level $j$ containing $v$ (if one exists).

Next, still for correct instances, we would like to represent the selected outgoing edges distributively. That is, each node $v$ should be able to detect, for each fragment $F$ containing $v$, whether $v$ is an endpoint of the selected edge of $F$. If $v$ is, it should also know which of $v$ 's edges is the selected edge. This representation is used later for verifying the two items of the Well-Forming property specified above. For this purpose, first, we add another string of $\ell+1$ entries at each node $v$, one entry per level $j$. This entry specifies, first, whether there exists a level $j$ fragment $F_{j}(v)$ containing $v$. If $F_{j}(v)$ does exist, the entry specifies whether $v$ is incident to the corresponding selected edge. Note, storing the information at $v$ specifying the pointers to all the selected edges of the fragments containing it, may require $O\left(\log ^{2} n\right)$ bits of memory at $v$. This is because there may be $O(\log n)$ fragments containing $v$; each of those may select an edge at $v$ leading to an arbitrary neighbour of $v$ in the tree $T$; if $v$ has many neighbours, each edge may $\operatorname{cost} O(\log n)$ bits to encode. The trick is to distribute the information regarding $v$ 's selected edges among $v$ 's children in $T$. (Recall that $v$ can look at the data structures of $v$ 's children.)

The strings mentioned in the previous paragraphs are supposed to define a hierarchy and selected outgoing edges from the fragments of the hierarchy. However, on incorrect instances, if corrupted, the strings may not 
represent the required. For example, the strings may represent more than one selected edge for some fragment. Hence, we need also to attach proof labels for verifying the hierarchy and the corresponding selected edges represented by those strings. Fortunately, for proving the Well-Forming property only, it is not required to verify that the represented hierarchy (and the corresponding selected edges) actually follow the MST construction algorithm (which is the case for correct instances). In Section 5, we present 1-proof labeling schemes to show that the above strings represent some hierarchy with corresponding selected edges, and that the Well-Forming property does hold for that hierarchy.

\subsection{Verifying The Minimality property (described in detail in Sections 6, 7 and 8 )}

A crucial point in the scheme is letting each node $v$ know, for each of its incident edges $(v, u) \in E$ and for each level $j$, whether $u$ and $v$ share the same level $j$ fragment. Intuitively, this is needed in order to identify outgoing edges. For that purpose, we assign each fragment a unique identifier, and $v$ compares the identifier of its own level $j$ fragment to the identifier of $u$ 's level $j$ fragment.

Consider the number of bits required to represent the identifiers of all the fragments that a node $v$ participates in. There exists a method to assign unique identifiers such that this total number is only $O(\log n)$ [56, 36]. Unfortunately, we did not manage to use that method here. Indeed, there exists a marker algorithm that assigns identifiers according to that method. However, we could not find a low space and short time method for the verifier to verify that the given identifiers of the fragments were indeed assigned in that way. (In particular, we could not verify efficiently that the given identifiers are indeed unique).

Hence, we assign identifiers according to another method that appears more memory wasteful, where the identity $\operatorname{ID}(F)$ of a fragment $F$ is composed of the (unique) identity of its root together with its level. We also need each node $v$ to know the weight $\omega(F)$ of the minimum outgoing edge of each fragment $F$ containing $v$. To summarize, the piece of information $\mathrm{I}(F)$ required in each node $v$ per fragment $F$ containing $v$ is $\operatorname{ID}(F) \circ \omega(F)$. Thus, $I(F)$ can be encoded using $O(\log n)$ bits. Again, note that since a node may participate in $\ell=\Theta(\log n)$ fragments, the total number of bits used for storing all the $\mathrm{I}(F)$ for all fragments $F$ containing $v$ would thus be $\Theta\left(\log ^{2} n\right)$. Had no additional steps been taken, this would have violated the $O(\log n)$ memory constraint.

Luckily, the total number of bits needed globally for representing the pieces $I(F)$ of all the fragments $F$ is only $O(n \log n)$, since there are at most $2 n$ fragments, and $\mathrm{I}(F)$ of a fragment $F$ is of size $O(\log n)$ bits. The difficulty results from the fact that multiple nodes need replicas of the same information. (E.g., all the nodes in a fragment need the ID of the fragment.) If a node does not store this information itself, it is not clear how to bring all the many pieces of information to each node who needs them, in a short time (in spite of congestion) and while having only a constant number of such pieces at a node at each given point in time.

To allow some node $v$ to check whether its neighbour $u$ belongs to $v$ 's level $j$ fragment $F_{j}(v)$ for some level $j$, the verifier at $v$ needs first to reconstruct the piece of information $\mathrm{I}\left(F_{j}(v)\right)$. Intuitively, we had to distribute the information, so that $\mathrm{I}(F)$ is placed "not too far" from every node in $F$. To compare $\mathrm{I}\left(F_{j}(v)\right)$ with a neighbour $u$, node $v$ must also obtain $\mathrm{I}\left(F_{j}(u)\right)$ from $u$. This requires some mechanism to synchronize the reconstructions in neighbouring nodes. Furthermore, the verifier must be able to overcome difficulties resulting from faults, which can corrupt the information stored, as well as the reconstruction and the synchronization mechanisms.

The above distribution of the I's is described in Section 6. The distributed algorithm for the "fragment by fragment" reconstruction (and synchronization) is described in Section7. The required verifications for validating the I's and comparing the information of neighbouring nodes are described in Section 8. 


\subsubsection{Distribution of the pieces of information (described in detail in Section 6 )}

At a very high level description, each node $v$ stores permanently $\mathrm{I}(F)$ for a constant number of fragments $F$. Using that, $\mathrm{I}(F)$ is "rotated" so that each node in $F$ "sees" $I(F)$ in $O(\log n)$ time. We term the mechanism that performs this rotation a train. A first idea would have been to have a separate train for each fragment $F$ that would "carry" the piece $I(F)$ and would allow all nodes in $F$ to see it. However, we did not manage to do that efficiently in terms of time and of space. That is, one train passing a node could delay the other trains that "wish" to pass it. Since neighbouring nodes may share only a subset of their fragments, it is not clear how to pipeline the trains. Hence, those delays could accumulate. Moreover, as detailed later, each train utilizes some (often more than constant) memory per node. Hence, a train per fragment would have prevented us from obtaining an $O(\log n)$ memory solution.

A more refined idea would have been to partition the tree into connected parts, such that each part $P$ intersects $O(|P|)$ fragments. Using such a partition, we could have allocated the $O(|P|)$ pieces (of these $O(|P|)$ fragments), so that each node of $P$ would have been assigned only a constant number of such pieces, $\operatorname{costing} O(\log n)$ bits per node. Moreover, just one train per part $P$ could have sufficed to rotate those pieces among the nodes of $P$. Each node in $P$ would have seen all the pieces $I(F)$ for fragments $F$ containing it in $O(|P|)$ time. Hence, this would have been time efficient too, had $P$ been small.

Unfortunately, we did not manage to construct the above partition. However, we managed to obtain a similar construction: we construct two partitions of $T$, called Top and Bottom. We also partitioned the fragments into two kinds: top and bottom fragments. Now, each part $P$ of partition Top intersects with $O(|P|)$ top fragments (plus any number of bottom fragments). Each part $P$ of partition Bottom intersects with $O(|P|)$ bottom fragments (plus top fragments that we do not count here). For each part in Top (respectively Bottom), we shall distribute the information regarding the $O(|P|)$ top (respectively, bottom) fragments it intersects with, so that each node would hold at most a constant number of such pieces of information. Essentially, the pieces of information regarding the corresponding fragments are put in the nodes of the part (permanently) according to a DFS (Depth First Search) order starting at the root of the part. For any node $v$, the two parts containing it encode together the information regarding all fragments containing $v$. Thus, to deliver all relevant information, it suffices to utilize one train per part (and hence, each node participates in two trains only). Furthermore, the partitions are made so that the diameter of each part is $O(\log n)$, which allows each train to quickly pass in all nodes, and hence to deliver the relevant information in short time.

The distributed implementation of this distribution of pieces of information, and, in particular, the distributed construction of the two partitions, required us to come up with a new multi-wave primitive, enabling an efficient (in $O(n))$ time) parallel (i.e., pipelined) executions of Wave\&Echo operations on all fragments of Hierarchy $\mathcal{H}_{\mathcal{M}}$.

\subsubsection{Viewing the pieces of information (described in detail in Section 7)}

Consider a node $v$ and a fragment $F_{j}(v)$ of level $j$ containing it. Recall that the information $\mathrm{I}\left(F_{j}(v)\right)$ should reside in some node of a part $P$ to which $v$ belongs. To allow $v$ to compare $\mathrm{I}\left(F_{j}(v)\right)$ to $\mathrm{I}\left(F_{j}(u)\right)$ for a neighbour $u$, both these pieces must somehow be "brought" to $v$. The process handling this task contains several components. The first component is called the train which is responsible for moving the pieces of information through $P$ 's nodes, such that each node does not hold more than $O(\log n)$ bits at a time, and such that in short time, each node in $P$ "sees" all pieces, and in some prescribed order. Essentially, a train is composed of two ingredients. The first ingredient called convergecast pipelines the pieces of information in a DFS order towards the root of the part (recall, the pieces of information of the corresponding fragments are initially located according to a DFS order). The second ingredient broadcasts the pieces from the root of the part to all nodes in the part. Since the number of pieces is $O(\log n)$ and the diameter of the part is $O(\log n)$, the synchronous environment guarantees 
that each piece of information is delivered to all nodes of a part in $O(\log n)$ time. On the other hand, in the asynchronous environment some delays may occur, and the delivery time becomes $O\left(\log ^{2} n\right)$. These time bounds are also required to self-stabilize the trains, by known art, see, e.g. [23, 20].

Unfortunately, delivering the necessary pieces of information at each node is not enough, since $\mathrm{I}\left(F_{j}(v)\right)$ may arrive at $v$ at a different time than $\mathrm{I}\left(F_{j}(u)\right)$ arrives at $u$. Recall that $u$ and its neighbour $v$ need to have these pieces simultaneously in order to compare them (to know whether the edge $e=(u, v)$ is outgoing from $F_{j}(v)$ ).

Further complications arise from the fact that the neighbours of a node $v$ may belong to different parts, so different trains pass there. Note that $v$ may have many neighbours, and we would not want to synchronize so many trains. Moreover, had we delayed the train at $v$ for synchronization, the delays would have accumulated, or even would have caused deadlocks. Hence, we do not delay these trains. Instead, $v$ repeatedly samples a piece from its train, and synchronizes the comparison of this piece with pieces sampled by its neighbours, while both trains advance without waiting. Perhaps not surprisingly, this synchronization turns out to be easier in synchronous networks, than in asynchronous ones. Our synchronization mechanism guarantees that each node can compare all pieces $\mathrm{I}\left(F_{j}(v)\right)$ with $\mathrm{I}\left(F_{j}(u)\right)$ for all neighbours $u$ and levels $j$ in a short time. Specifically, $O\left(\log ^{2} n\right)$ time in synchronous environments and $O\left(\Delta \log ^{3} n\right)$ time in asynchronous ones.

\subsubsection{Local verifications (described in detail in Section 8)}

So far, with respect to verifying the Minimality property, we have not discussed issues of faults that may complicate the verification. Recall, the verification process must detect if the tree is not an MST. Informally, this must hold despite the fact that the train processes, the partitions, and also, the pieces of information carried by the trains may be corrupted by an adversary. For example, the adversary may change or erase some (or even all) of such pieces corresponding to existing fragments. Moreover, even correct pieces that correspond to existing fragments may not arrive at a node in the case that the adversary corrupted the partitions or the train mechanism.

In Section 8, we explain how the verifier does overcome such undesirable phenomena, if they occur. Intuitively, what is detected is not necessarily the fact that a train is corrupted (for example). Instead, what is detected is the state that some part is incorrect (either the tree is not an MST, or the train is corrupted, or ... etc.). Specifically, we show that if an MST is not represented in the network, this is detected in time $O\left(\log ^{2} n\right)$ for synchronous environments and time $O\left(\Delta \log ^{3} n\right)$ for asynchronous ones. Note that for a verifier, the ability to detect while assuming any initial configuration means that the verifier is self-stabilizing, since the sole purpose of the verifier is to detect.

Verifying that some two partitions exist is easy. However, verifying that the given partitions are as described in Section 6.1, rather than being two arbitrary partitions generated by an adversary seems difficult. Fortunately, this verification turns out to be unnecessary.

First, as mentioned, it is a known art to self-stabilize the train process. After trains stabilize, we verify that the set of pieces stored in a part (and delivered by the train) includes all the (possibly corrupted) pieces of the form $\mathrm{I}\left(F_{j}(v)\right.$ ), for every $v$ in the part and for every $j$ such that $v$ belongs to a level $j$ fragment. Essentially, this is done by verifying at the root $r(P)$ of a part $P$, that (1) the information regarding fragments arrives at it in a cyclic order (the order in which pieces of information are supposed to be stored in correct instances), (2) the levels of pieces arriving at $r(P)$ comply with the levels of fragments to which $r(P)$ belongs to, as indicated by $r(P)$ 's data-structure. Next, we verify that the time in which each node obtains all the pieces it needs is short. This is guaranteed by the correct train operation, as long as the diameter of parts is $O(\log n)$, and the number of pieces stored permanently at the nodes of the part is $O(\log n)$. Verifying these two properties is accomplished using a 1-proof labelling scheme of size $O(\log n)$, similarly to the schemes described in Examples 2 and 3 (SP and EDIAM, mentioned in Section 2.6. 
Finally, if up to this point, no node raised an alarm, then for each node $v$, the (possibly corrupted) pieces of information corresponding to $v$ 's fragments reach $v$ in the prescribed time bounds. Now, by the train synchronization process, each node can compare its pieces of information with the ones of its neighbours. Hence, using similar arguments as was used in the $O\left(\log ^{2} n\right)$-memory bits verification scheme of [55], nodes can now detect the case that either one of the pieces of information is corrupted or that $T$ is not an MST.

\section{A synchronous MST construction in $O(\log n)$ bits memory size and $O(n)$ time}

In this section, we describe an MST construction algorithm, called SYNC_MST, that is both linear in its running time and memory optimal, that is, it runs in $O(n)$ time and has $O(\log n)$ memory size. We note that this algorithm is not self-stabilizing and its correct operation assumes a synchronous environment. The algorithm will be useful later for two purposes. The first is for distributively assigning the labels of the MST proof labelling scheme, as described in the next section. The second purpose, is to be used as a module in the self-stabilizing MST construction algorithm.

As mentioned, the algorithm of Gallager, Humblet, and Spira (GHS) [40] constructs an MST in $O(n \log n)$ time. This has been improved by Awerbuch to linear time, using a somewhat involved algorithm. Both algorithms are also efficient in terms of the number of messages they send. The MST construction algorithm described in this section is, basically, a simplification of the GHS algorithm. There are two reasons for why we can simplify that algorithm, and even get a better time complexity. The first reason is that our algorithm is synchronous, whereas GHS (as well as the algorithm by Awerbuch) is designed for asynchronous environments. Our second aid is the fact that we do not care about saving messages (anyhow, we use a shared memory model), while the above mentioned algorithms strive to have an optimal message complexity. Before describing our MST construction algorithm, we recall the main features of the GHS algorithm.

\subsection{Recalling the MST algorithm of Gallager, Humblet, and Spira (GHS)}

For full details of GHS, please refer to [40]. GHS uses connected subgraphs of the final MST, called fragments. Each node in a fragment, except for the fragment's root, has a pointer to its parent in the fragment. When the algorithm starts, every node is the root of the singleton fragment including only itself. Each fragment is associated with its level (zero for a singleton fragment) and the identity of its root (this is a slight difference from the description in [40], where a fragment is said to be rooted at an edge). Each fragment $F$ searches for its minimum outgoing edge $e_{\min }(F)=(v, u)$. Using the selected edges, fragments are merged to produce larger fragments of larger levels. That is, two or more fragments of some level $j$, possibly together with some fragments of levels lower than $j$, are merged to create a fragment of level $j+1$. Eventually, there remains only one fragment spanning the graph which is an MST.

In more details, each fragment sends an offer (over $e_{\min }(F)$ ) to merge with the other fragment $F^{\prime}$, to which the other endpoint $u$ belongs. If $F^{\prime}$ is of a higher level, then $F$ is connected to $F^{\prime}$. That is, the edges in $F$ are reoriented so that $F$ is now rooted in the endpoint $v$ of $e_{\min }(F)$, which becomes a child of the other endpoint $u$.

If the level of $F^{\prime}$ is lower, then $F$ waits until the level of $F^{\prime}$ grows (see below, the description of "test" messages). The interesting case is when $F$ and $F^{\prime}$ are of the same level $j$. If $e_{\min }(F)=e_{\min }\left(F^{\prime}\right)$, then $F$ and $F^{\prime}$ merge to become one fragment, rooted at, say, the highest ID node between $u$ and $v$. The level of the merged fragment is set to $j+1$.

The remaining case, that (w.l.o.g.) $w\left(e_{\min }(F)\right)>w\left(e_{\min }\left(F^{\prime}\right)\right)$ does not need a special treatment. When $F$ sends $F^{\prime}$ an offer to merge, $F^{\prime}$ may have sent such an offer to some $F^{\prime \prime}$ over $w\left(e_{\min }\left(F^{\prime}\right)\right)$. Similarly, $F^{\prime \prime}$ may have sent an offer to some $F^{\prime \prime \prime}$ (over $w\left(e_{\min }\left(F^{\prime \prime}\right)\right)$ ), etc. No cycle can be created in this chain of offers (because 
of the chain of decreasing weights $\left.w\left(e_{\min }(F)\right)>w\left(e_{\min }\left(F^{\prime}\right)\right)>w\left(e_{\min }\left(F^{\prime \prime}\right)\right) \ldots\right)$. Hence, unless the chain ends with some fragment of a higher level (recall that treating the case that a fragment's minimum edge leads to a higher level fragment was already discussed), some two fragments in the above chain merge, increasing their level by one. This case (for the fragments of the chain, excluding the two merging fragments) now reduces to the case (discussed previously) that a fragment $F$ makes an offer to a fragment of a higher level.

The above describes the behavior of fragments. To implement it by nodes, recall that every fragment always has a root. The root conducts Wave\&Echo over the fragment to ask nodes to find their own candidate edges for the minimum outgoing edge. On receiving the wave (called "find"), each node $v$ selects its minimum edge $(v, u)$ that does not belong yet to the fragment, and has not been "tested" yet (initially, no edge was "tested"). Node $v$ sends a "test" message to $u$, to find out whether $u$ belongs to $v$ 's fragment. The "test" includes the ID of $v$ 's fragment's root $r$ and its level $j$. If the level of $u$ 's fragment is at least $j$ then $u$ answers. In particular, if $u$ 's level is $j$ and $u$ 's fragment root is $r$ then $u$ sends a "reject" to $v$, causing $v$ to conclude that $(v, u)$ is not outgoing and cannot be a candidate. (Node $u$ does not answer, until its level reaches $j$, thus, possibly, causing $v$ 's fragment to wait.) In the converging wave (called "found") of the above "find" broadcast, each node $v$ passes to its parent only the candidate edge with the minimum weight (among its own candidate and the candidates it received from its children). Node $v$ also remembers a pointer to whoever sent it the above candidate. These pointers form a route from $F^{\prime} s$ root to the endpoint of $e_{\min }(F)$. The root then sends a message "change-root", instructing all the nodes on this route (including itself) to reverse their parent pointers. Hence, $F$ becomes rooted at the endpoint of $e_{\min }(F)$, who now can send an offer to "connect" over $e_{\min }(F)$.

\subsection{Algorithm SYNC_MST: a synchronous linear time version with optimal memory size}

The algorithm we now describe is synchronous and assumes that all the nodes wake up simultaneously at round 0 . However, to keep it easy for readers who are familiar with GHS, we tried to keep it as similar to GHS as possible.

Initially, each node is a root of a fragment of level 0 that contains only itself. During the execution of SYNC_MST, a node who is not a root, keeps a pointer to its parent. The collection of these pointers (together with all the nodes) defines a forest at all times. Each node also keeps an estimate of the ID and the level of the root of its fragment. As we shall see later, the ID estimate is not always accurate. The level estimate is a lower bound on the actual level. We use the levels for convenience of comparing the algorithm to that of GHS (and for the convenience of the proof). The levels actually can be computed from the round number, or from the counting procedure defined below. More specifically, the algorithm is performed in synchronous phases. Phase $i$ starts at round $11 \cdot 2^{i}$. Each root $r(F)$ of a fragment $F$ (that is, a node whose parent pointer is null) starts the phase by setting its level to $i$ and then counting the number of nodes in its fragment.

The counting process, called Count_Size, is defined later, but for now it suffices to say that it consumes precisely $2^{i+2}-1$ rounds. If the diameter of the fragment is small, then some waiting time is added to keep the precise timing. On the other hand, if the number of nodes in the fragment is too large, Count_Size may terminate before all the nodes in the fragment are counted. Specifically, we guarantee that if the counting process succeeds to count all nodes in the fragment $F$ then the precise number of these nodes is known to the root $r(F)$ at the end of the counting procedure. On the other hand, if the counting process does not count all nodes, then the number of nodes in the fragment is at least $2^{i+1}$, and at the end of the Count_Size process, the root $r(F)$ learns this fact. Moreover, in such a case, as a consequence, $r(F)$ changes its level to $i+1$.

Definition 4.1 A root $r(F)$ is active in phase $i$ if and only if $|F| \leq 2^{i+1}-1$, where $|F|$ denotes the number of nodes in $F$. Note that if $r(F)$ is active then its level is $i$. In particular, all the roots are active in phase (and level) 0 . A fragment is active when its root is active. 
Comment 4.1 When constructing the marker algorithm in later sections, we use the fragments constructed by algorithm SYNC_MST. More specifically, we refer only to the active fragments. As is easy to observe below in the current section, an active fragment is a specific set of nodes that does not change. This is because when the fragment merges with others (or when others join it), it is no longer the same fragment. In particular, when the new set of nodes will be active, it will be in a higher phase.

Procedure Find_Min_Out_Edge: Consider the root $r(F)$ of fragment $F$, who is active in phase $i$. At round $(11+4) \cdot 2^{i}$, each such root $r(F)$ instructs the nodes in $F$ to search for the minimum outgoing edge of $F$. This procedure, called Find_Min_Out_Edge, could have been combined with the counting, but we describe it as a separate stage for the sake of simplicity. The method is the same as that of GHS algorithm, except that we achieve an exact timing obtained by not saving in messages. The search is performed over exactly the same set of nodes which has just been counted. This is implemented by a Wave operation initiated by $r(F)$, carrying $r(F)$ 's ID and level. At precisely round $(11+6) \cdot 2^{i}$, each node $v$ who has received the wave, finds the minimum outgoing edge emanating from it. That is, $v$ looks at each of its neighbours $u$ to see whether $u$ belongs to a different fragment of some other root $r\left(F^{\prime}\right) \neq r(F)$. We now describe how $v$ identifies this.

Let us note here two differences from GHS. First, node $v$ tests all of its emanating edges at the same time, rather than testing them one by one (as is done in GHS). Moreover, it does not reject any edge, and will test all its edges in the next searches too. Intuitively, the above mentioned one by one process was used in GHS in order to save messages. We do not try to save messages, and the simultaneous testing allows us to keep an exact timing on which we rely heavily. Second, in GHS, node $u$ 's estimate of its level may be lower than that of node $v$. In GHS, $v$ then needs to wait for $u$ to reach $v$ 's level, before $v$ knows whether edge $(v, u)$ is outgoing. The main reason this action is useful in GHS is to save on message complexity. Here, again, we do not intend to save messages.

Recall that the root of $v$ 's fragment $F$ is active at phase $i$, hence, $|F|<2^{i+1}$. (We shall show that no additional nodes joined $F$ after the counting.) Hence, at round $(11+6) \cdot 2^{i}$, all the nodes in $F$ have already received the wave, and set their ID estimates to $\operatorname{ID}(r(F))$. The big gain from that, is that at round $(11+6) \cdot 2^{i}$, the IDs of the roots of $u$ and $v$ are different if and only if the edge $(v, u)$ is outgoing at $v$. The minimum outgoing edge in the fragment of $r(F)$ is then computed during the convergecast, using the standard Wave\&Echo technique. Thus, Procedure F ind_Min_Out_Edge (composed of the aforementioned Wave\&Echo) lasts at most $2\left(2^{i+1}-1\right)$ round units, hence (having been started at round $\left.(11+4) \cdot 2^{i}\right)$, it is completed by round $(11+8) \cdot 2^{i}-1$.

Merging and reorienting edges: Let $(w, x)$ be the chosen minimum outgoing edge from the fragment $F$, such that $w \in F$. Later, we refer to it as the candidate edge. At round $(11+8) \cdot 2^{i}$, an active $\operatorname{root} r(F)$ of $F$ starts the process of re-orienting the edges in $F$ towards $w$. (For a more thorough description of the root transfer refer to [40].) This takes at most $2\left(2^{i}-1\right)$ rounds.

Node $w$ then conducts a handshake with $x$, referred to as the pivot of $F$. This takes a constant time, but, to keep the total numbers simple, we pad this time to $2^{i}$. One case is that $w$ is, at that time exactly, a pivot of the fragment of $x$, and also $\operatorname{ID}(x)<\operatorname{ID}(w)$. In this case, node $x$ will become the child of $w$. In every other case, $w$ hooks upon the other endpoint $x$ (sets its parent pointer to point at $x$ ). The hooking is performed exactly at round $(11+11) \cdot 2^{i}-1$, ending phase $i$. Since the next phase starts at round $22 \cdot 2^{i}$ there is no overlap between phases.

Procedure Count_Size: To complete the description of a phase, it is left to describe the counting process, namely, Procedure Count_Size. To count, a root starts a Wave\&Echo, attaching a time-to-live $=2^{i+1}-1$ counter to its broadcast message. A child $c$ of a node $y$ accepts the wave only if the time-to-live is above zero. Child $c$ then copies the wave broadcast message, decrementing the time-to-live (by 1). If, after decrementing, the value of time-to-live is zero, then $c$ is a leaf who needs to start the echo. The number of the nodes (who copied 
the broadcast message) is now counted during the echo in the standard way. Finally, if the count covers the whole graph, this can be easily detected at the time of the echo. The algorithm then terminates.

To sum up, phase $i$ of the MST construction algorithm is composed of the following components.

\section{$\underline{\text { Phase } i}$}

- Starts at round $11 \cdot 2^{i}$;

- Root $r(F)$ of each fragment $F$ initiates Procedure Count_Size. At the end of the procedure, we have: $|F| \leq 2^{i+1}-1$ iff (1) $r(F)$ is active and (2) all nodes in $F$ have their ID estimates set to $\operatorname{ID}(r(F))$;

- At round $(11+4) \cdot 2^{i}$, each active root $r(F)$ initiates Procedure Find_Min_Out_Edge ;

- At round $(11+8) \cdot 2^{i}$, merge fragments and re-orient edges in the newly created fragments.

The proof that the collection of parent pointers forms a forest (or a tree) at all times is the same as in GHS. Let us now analyze the round complexity. Observe that each phase $i$ takes $O\left(2^{i}\right)$ time. Hence, the linear time complexity of the algorithm follows from the lemma below.

Lemma 4.1 The size of a fragment $F$ in phase $i$ (and in level $i$ ) is at least $2^{i}$. Moreover, $|F|<2^{i+1}$ if and only if $r(F)$ is active by round $(11+4) \cdot 2^{i}$.

Proof: Let us first prove the second part of the lemma. Before deciding whether to be active, a root $r(F)$ of level $i$ counts the number of nodes in its fragment, by employing Procedure Count_Size. If the count amounts to $2^{i+1}$ or more, then the level of $r(F)$ is set to $i+1$. Otherwise, we have $|F| \leq 2^{i+1}-1$ and the root of $F$ becomes active. Since Procedure Count_Si ze is terminated by round $(11+4) \cdot 2^{i}$, the second part of the lemma follows. To prove the first part of the lemma, we need to show that the size of a level $i$ fragment is at least $2^{i}$. We prove this by induction on $i$.

Intuitively, the induction says that each fragment at the beginning of phase $i-1$, is of size at least $2^{i-1}$. During phase $i-1$, by the second part of the lemma, at time $(11+4) \cdot 2^{i-1}$, all the non-active fragments are already of size at least $2^{i}$ and are also of level $i$ (as a result of the count). As for active fragments, each such fragment is combined to at least one other fragment, so the resulting size is at least $2 \cdot 2^{i-1}=2^{i}$.

In more details, note that the claim holds for phase $i=0$. For a larger phase $i$, assume that the lemma holds for phases up to $i-1$ including. Consider a root $r(F)$ of a fragment $F$ of level $i$. It was a root also at level $i-1$. First, assume that at phase $i-1$, some other root $r\left(F_{1}\right)$ hooked upon $r(F)$ 's tree. To do so, $r\left(F_{1}\right)$ had to be active at phase $i-1$. By the induction hypothesis (the first part of the lemma), the size of fragment $F_{1}$, as well as the size of fragment of $F$ at that point in time, was at least $2^{i-1}$. The claim, in this case, follows.

Now, assume that no other fragment hooked upon fragment $F$ in phase $i-1$. Note that $F$ at level $i-1$ does not span the graph (otherwise, no root would reach level $i$, by the 2nd part of the inductive hypothesis, and since the counting process on trees is a known art and is known to be correct). Hence, it has a minimum outgoing edge $e=(w, x)$, where $w \in F$ and $x$ belongs to some other fragment $F_{x}$. We claim that the search process Find_Min_Out_Edge does find that edge $e$. Recall that in the fragment of an active root, the counting reaches all the nodes in the fragment. Hence, each of them knows the ID of their root $r(F)$ at the time the search in their fragment starts. Moreover, since a hooking is performed only at times of the form $(11+11) \cdot 2^{j}-1$, no new nodes (or fragments) join until the last time step of the phase (which is after the search, because of what we established about the size of the fragment). 
We claim that either (a) Edge $e=(w, x)$ was not the minimum outgoing edge of $x$ 's fragment $F_{x}$, or, alternatively, (b) the root $r_{x}$ of $x$ 's fragment $F_{x}$ had level $i$ at that time, or (c) $\operatorname{ID}(x)>\operatorname{ID}(w)$. Assume the contrary. By the inductive hypothesis, node $r_{x}$ is at least at level $i-1$. Since we assumed that (b) does not hold, $r_{x}$ is exactly at level $i-1$. This means (by the correctness of the counting) that $r_{x}$ is active at phase $i-1$. Similarly, this also means that the size of $F_{x}$ is less than $2^{i}$. Hence, and by the induction hypothesis, the counting, the searching, the root transfer, and the handshake end in $x$ 's fragment at the same time they end in $w$ 's fragment. (These processes use known art, and we shall not prove them here.) Then $x$ hooks upon $w$, contrary to our assumption.

We have just established that the conditions for $w$ to hook upon $x$ hold. Hence, $w$ hooks upon $x$. Similarly to the previous case, the size of w's fragment, as well as the size of $x$ 's fragments at that point in time is at least $2^{i-1}$. Thus, the size of the combined fragment is at least $2^{i}$. This concludes the proof of the first part of the lemma.

Corollary 4.2 The synchronous MST construction algorithm computes an MST in time $O(n)$.

Implementing the algorithm in the shared memory model with $O(\log n)$ memory: Each node $v$ keeps its fragment level and root ID. Node $v$ also remembers whether $v$ is in the stage of counting the number of nodes, or searching for the minimum outgoing edge. It also needs to remember whether it is in the wave stage, or has already sent the echo. Node $v$ needs to remember the candidate (for being $e_{\min }(F)$ ) edge that $v$ computed in the echo ("found") stage of the convergecast. If this candidate was reported by a child, then $v$ also remembers a pointer to that child. Clearly, all the above variables combined need $O(\log n)$ bits of memory.

At a first glimpse it may look as if a node must also remember the list of pointers to its children. The list is used for (1) sending the wave (e.g., the "find" message of the search, using GHS terms) to all the children, and (2) knowing when all the children answered the echo (e.g., the "found" of the search). Note that a node does not need to store this list itself. Node $v$ can look at each neighbour $u$ to see whether the neighbour is $v$ 's child. (For that purpose, if $u$ is a child of $v$, then $u$ stores $v$ 's ID as $u$ 's Parent_ID.) Clearly, this can be implemented using $O(\log n)$ bits per node.

To implement (1), node $v$ posts its wave broadcast message (e.g., the "find") so that every neighbour can read it. However, only its children actually do. To allow the implementation of (2), a precaution is taken before the above posting. Node $v$ first posts a request for its children to reset their ECHO variables, and performs the posting of "find" only when it sees that $\mathrm{ECHO}$ has been reset for every neighbour $w$ whose parent pointer points at $v$.

To implement (2), node $v$ further reads its neighbours repeatedly. It knows all its children echoed the wave in an iteration when it has just finished rereading all its neighbours, and every node $u$ pointing at $v$ (as its parent), also sets its $\mathrm{ECHO}$ variable to some candidate edge (or to some default value if it has no candidate edge).

Observation 4.3 The space required by the linear time synchronous algorithm is $O(\log n)$ bits per node.

The theorem below follows from Observation 4.3 and Corollary 4.2

Theorem 4.4 The synchronous algorithm SYNC_MST computes an MST in time $O(n)$ and memory size $O(\log n)$. 


\section{Representing and verifying a hierarchy}

We are now ready to describe our proof labeling scheme $(\mathcal{M}, \mathcal{D})$ for MST. The goal of this section is to construct some part of the marker $\mathcal{M}$, and the corresponding part of the verifier $\mathcal{D}$, which are relatively easy to construct. The techniques used in this section bear similarity to the techniques presented in [55]. Hence, we only expose the main ideas behind this part of the proof labeling scheme, leaving out some of the technicalities. Nevertheless, since the notion of proof labeling schemes can sometimes be confusing, this section may help the reader to get accustomed to the notion and the difficulties that may arise.

As a warm up, we first note that using the 1-proof labeling scheme described in Example SP, we may assume that $H(G) \equiv T$ is a spanning tree of $G$ rooted at some node $r$, and that each node knows which of its neighbours in $G$ are its children in $T$ and which is its parent. Moreover, using the 1-proof labeling scheme described in Example NumK, we may also assume that each node knows $n$. The 1-proof labeling schemes described in Examples SP and NumK use $O(\log n)$ memory size and can be constructed using $O(n)$ time. Hence, using them does not violate the desired complexity constrains of our scheme. Thus, from now on, let us fix a spanning tree $T=(V(G), E(T))$ of a graph $G=(V(G), E(G))$, rooted at some node $r(T)$. The goal of the rest of the verification scheme is to verify that $T$ is in fact, minimal. Before we continue, we need a few definitions.

Definition $5.1 A$ hierarchy $\mathcal{H}$ for $T$ is a collection of fragments of $T$ satisfying the following two properties.

1. $T \in \mathcal{H}$ and, for every $v \in V(G)$, there is an $F_{v} \in \mathcal{H}$ such such $V\left(F_{v}\right)=\{v\}$ and $E\left(F_{v}\right)=\emptyset$.

2. For any two fragments $F$ and $F^{\prime}$ in $\mathcal{H}$, if $F \cap F^{\prime} \neq \emptyset$ then either $F \subseteq F^{\prime}$ or $F^{\prime} \subseteq F$. (That is, the collection of fragments is a laminar family.)

Please recall (Definition 4.1 and Comment 4.1) that when we construct a hierarchy according to Definition 5.1, the fragments referred to are the active fragments constructed in SYNC_MST.

The root of a fragment $F$ is the node in $F$ closest to the root of $T$. For a fragment $F \in \mathcal{H}$, let $\mathcal{H}(F)$ denote the collection of fragments in $\mathcal{H}$ which are strictly contained in $F$. Observe that a hierarchy $\mathcal{H}$ can be viewed as a rooted tree, whose root is the fragment $T$, and whose leaves are the singleton fragments in $\mathcal{H}$. A child of a nonsingleton fragment $F \in \mathcal{H}$ is a fragment $F^{\prime} \in \mathcal{H}(F)$ such that no other fragment $F^{\prime \prime} \in \mathcal{H}(F)$ satisfies $F^{\prime \prime} \supset F^{\prime}$. Note that the rooted tree induced by a hierarchy is unique (if the children are unordered). To avoid confusion with tree $T$, we use the name hierarchy-tree (or, sometimes even just hierarchy) for the above mentioned tree induced by a hierarchy. We associate a level, denoted $\operatorname{lev}(F)$, with each fragment $F \in \mathcal{H}$. It is defined as the height of the node corresponding to $F$ in the hierarchy-tree induced by $\mathcal{H}$, i.e., the maximal number of fragments on a simple path in $\mathcal{H}$ connecting $F$ to a singleton fragment. In particular, the level of a singleton fragment is 0. The level of the fragment $T$ is called the height of the hierarchy, and is denoted by $\ell$. Figure 1 depicts a hierarchy $\mathcal{H}$ of a tree $T$.

Definition 5.2 Given a hierarchy $\mathcal{H}$ for a spanning tree $T$, a function $\chi: \mathcal{H} \backslash\{T\} \longrightarrow E(T)$ is called a candidate function of $\mathcal{H}$ if it satisfies $E(F)=\left\{\chi\left(F^{\prime}\right) \mid F^{\prime} \in \mathcal{H}(F)\right\}$ for every $F \in \mathcal{H}$. (Less formally, $F$ is precisely the union of the candidate edges $\chi\left(F^{\prime}\right)$ of all fragments $F^{\prime}$ of $\mathcal{H}$ strictly contained in $\left.F\right)$.

The proof of the following lemma is similar, e.g., to the proof of [40].

Lemma 5.1 Let $T$ be a spanning tree of a graph $G$. If there exists a candidate function $\chi$ for a hierarchy $\mathcal{H}$ for $T$, such that for every $F \in \mathcal{H}$, the candidate edge $\chi(F)$ is a minimum outgoing edge from $F$, then $T$ is an MST of $G$. 


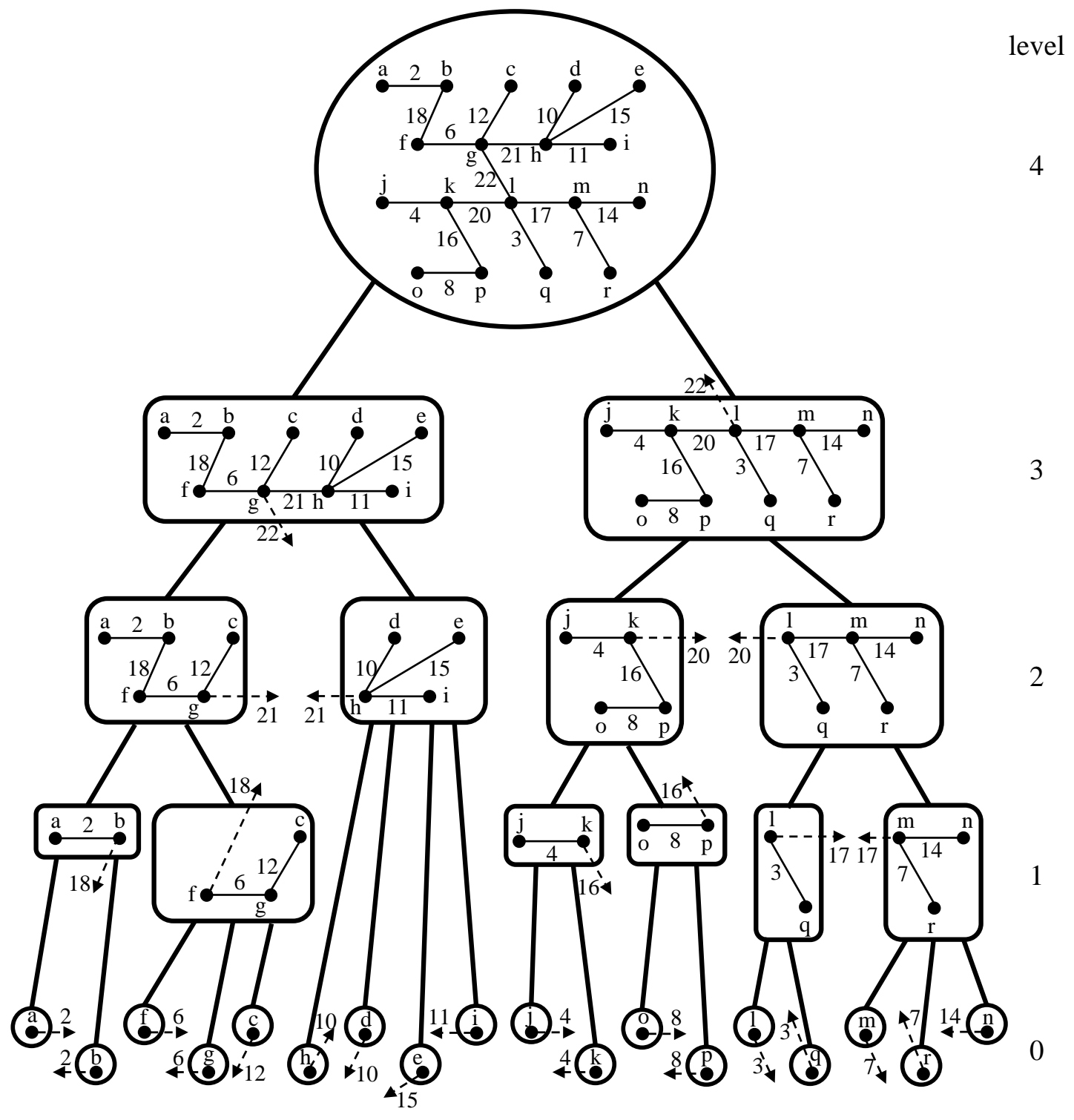

Figure 1: A hierarchy $\mathcal{H}$ of a tree $T$. The root node of $\mathcal{H}$ represents $T$ (where non-tree edges are omitted). Each fragment that is not a leaf fragment is a parent, in the hierarchy, of the fragments that were merged to form it. The broken arrow from each fragment is the outgoing edge of the fragment that is used to form a higher level (parent) fragment. 
Proof: We prove the claim that each fragment $F \in \mathcal{H}$ is a subtree of an MST of $G$, by induction on the level $\operatorname{lev}(F)$ of fragment $F$. Note that the claim obviously holds for any fragment $F$ with $\operatorname{lev}(F)=0$ since $F$ is a singleton fragment.

Now consider a fragment $F$ with $\operatorname{lev}(F)=k$ under the inductive assumption that the claim holds for every fragment $F^{\prime}$ with $\operatorname{lev}\left(F^{\prime}\right)<k$. Let $F_{1}, F_{2}, \ldots, F_{a}$ be the child fragments of $F$ in $\mathcal{H}$. Since for each $i \in[1, a]$, fragment $F_{i}$ satisfies $l e v\left(F_{i}\right)<k$, the induction hypothesis implies that $F_{i}$ is a subtree of the MST. It also follows from the facts that $E(F)=\left\{\chi\left(F^{\prime}\right) \mid F^{\prime} \in \mathcal{H}(F)\right\}$ and $E\left(F_{i}\right)=\left\{\chi\left(F^{\prime}\right) \mid F^{\prime} \in \mathcal{H}\left(F_{i}\right)\right\}$ for each $i \in[1, a]$ that the fragment $F$ is obtained by connecting $F_{1}, F_{2}, \ldots, F_{a}$ with their minimum outgoing edges. In the case that a fragment $F^{\prime}$ is a fragment of an MST (as is the case here for $F_{1}, F_{2}, \ldots, F_{a}$, by the induction hypothesis), it is known that the union of $E\left(F^{\prime}\right)$ with the minimum outgoing edge of $F^{\prime}$ is a fragment of the MST (the "safe edge" theorem). (See e.g., [35].) Thus, fragment $F$, which is obtained by connecting fragments $F_{1}, F_{2}, \ldots, F_{a}$ with their minimum outgoing edges, is a subgraph of an MST.

Informally, suppose that we are given distributed structures that are claimed to be a tree $T$, a "legal" hierarchy $\mathcal{H}$ for the tree, and a "legal" candidate function for the hierarchy. The goal obtained in the current section is to verify the following properties of these structures. First, verify that this indeed is a hierarchy for $T$ of height $\ell \leq\lceil\log n\rceil$ and a candidate function $\chi$ for $\mathcal{H}$. Moreover, verify that each node $v$ "knows" to which levels of fragments $v$ belongs and which of its neighbours in $T$ share the same given fragment. (Note that this section does not guarantee that knowledge for neighbours in $G$ who are not neighbours in $T$.) In addition, each node is verified to "know" whether it is adjacent to a candidate edge of any of the fragments it belongs to. Put more formally, this section establishes the following lemma.

Lemma 5.2 There exists a 1-proof labeling scheme with memory size $O(\log n)$ and construction time $O(n)$ that verifies the following:

- $H(G) \equiv T$ is a spanning tree of $G$ rooted at some node $r$, and each node knowns $n$.

- The cartesian product of the data-structures indeed implies a hierarchy $\mathcal{H}$ for $T$ of height $\ell \leq\lceil\log n\rceil$ and a candidate function $\chi$ for $\mathcal{H}$. Furthermore, the data-structure at each node $v$ allows it to know,

- Whether $v$ belongs to a fragment $F_{j}(v)$ of level $j$ in $\mathcal{H}$ for each $0 \leq j \leq \ell$, and if so:

- Whether $v$ is the root of $F_{j}(v)$.

- Whether $v$ is an endpoint of the (unique) candidate edge of $F_{j}(v)$, and if so, which of the edges adjacent to $v$ is the candidate edge.

- Given the data-structure of a node $u$ which is a neighbour of $v$ in $G$, i.e., $(v, u) \in E(G)$, node $v$ can find out whether they are neighbours in $T$ as well, i.e., whether $(u, v) \in E(T)$, and if so, for each $1 \leq j \leq \ell$, whether u belongs to $F_{j}(v)$.

\subsection{Hierarchy $\mathcal{H}_{\mathcal{M}}$ and candidate function $\chi_{\mathcal{M}}$}

On a correct instance, i.e., when $T$ is indeed an MST, the marker $\mathcal{M}$ first constructs a particular hierarchy $\mathcal{H}_{\mathcal{M}}$ over $T$ and a candidate function $\chi_{\mathcal{M}}$ for that hierarchy. Hierarchy $\mathcal{H}_{\mathcal{M}}$ and candidate function $\chi_{\mathcal{M}}$ are designed so that indeed each candidate of a fragment is a minimum outgoing edge from that fragment. The marker then encodes hierarchy $\mathcal{H}_{\mathcal{M}}$ and candidate function $\chi_{\mathcal{M}}$ in one designated part of the labels using $O(\log n)$ bits per node. Note, however, that these bits of information may be corrupted by the adversary. We will therefore need to employ another procedure that verifies that indeed a hierarchy $\mathcal{H}$ and a candidate function $\chi$ are represented 
by the cartesian product of the encodings of all nodes. By Lemma 5.1, it is not necessary that the verifier checks that $\mathcal{H}$ is, in fact, the particular hierarchy $\mathcal{H}_{\mathcal{M}}$ constructed by the marker, or that the candidate function $\chi$ is $\chi_{\mathcal{M}}$. However, as is clear from the same lemma, we do need to show that $\mathcal{H}$ and candidate function $\chi$ satisfy that indeed each candidate of a fragment is a minimum outgoing edge from that fragment. This task is the main technical difficulty of the paper, and is left for the following sections.

The hierarchy $\mathcal{H}_{\mathcal{M}}$ and Candidate function $\chi_{\mathcal{M}}$ built by the marker algorithm are based on SYNC_MST, the new MST construction algorithm described in Section 4. Since we assume that the MST is unique, Algorithm SYNC_MST will in fact construct the given MST. (Recall that we describe here the labels assigned by the marker to a correct instance, where the given subgraph $T$ is indeed an MST.) The hierarchy and candidate function we define for $T$ follow the merging of active fragments in algorithm SYNC_MST. More precisely, the nodes in $\mathcal{H}_{\mathcal{M}}$ are the active fragments defined during the execution of SYNC_MST. Recall from Section 4, that an active fragment $F$ joins some fragment $H$ of $T$, through its minimal outgoing edge $e$. (It is possible that at the time $F$ joins $H, H$ itself was an active fragment that joined $F$ through its own minimal outgoing edge that is also e.) Note that with time, some other fragments join the resulted connected component, until, at some point, the resulted connected component becomes an active fragment $F^{\prime}$. In the hierarchy tree $\mathcal{H}_{\mathcal{M}}$, fragment $F$ is defined as the child of $F^{\prime}$, and the candidate edge of $F$ is $e$, i.e., $\chi(F)=e$.

As proved in Lemma 4.1, after performing the algorithm for level $i$, the size of every fragment is at least $2^{i}$. Thus, in particular, the height of the hierarchy $\mathcal{H}$ is at most $\lceil\log n\rceil$. The candidate function $\chi_{\mathcal{M}}$ chosen by the marker for $\mathcal{H}_{\mathcal{M}}$ is defined by the minimum outgoing edges selected by the algorithm, i.e., for each $F \in \mathcal{H}_{\mathcal{M}}$, the candidate edge $\chi(F)$ is the selected edge of $F$. Thus, under $\chi_{\mathcal{M}}$, each candidate of a fragment is, actually, a minimum outgoing edge.

\subsection{Representing a hierarchy distributively and verifying it locally}

Representing a hierarchy: Let $\ell \leq\lceil\log n\rceil$. Given a hierarchy of fragments $\mathcal{H}$ of height $\ell$ over the rooted tree $T=H(G)$, we now describe how we represent it in a distributed manner. Each node $v$ keeps a string named $\operatorname{Roots}(v)$ of length $\ell+1$, where each entry in that string is either " 1 ", " 0 ", or "*". To be consistent with the levels, we enumerate the entries of each string from left to right, starting at position 0 , and ending at position $\ell$. Fix $j \in[0, \ell]$. Informally, the $i$ 'th entry of $\operatorname{Roots}(v)$, namely, $\operatorname{Roots}_{i}(v)$, is interpreted as follows.

- $\operatorname{Roots}_{i}(v)=1$ indicates that $v$ is the root of the level $i$ fragment it belongs to.

- $\operatorname{Root} \mathrm{s}_{i}(v)=0$ indicates that $v$ is not the root of the level $i$ fragment it belongs to.

- $\operatorname{Roots}_{i}(v)=*$ indicates that there is no level $i$ fragment that $v$ belongs to.

See Table 2 for an example of Roots strings of nodes corresponding to Figure 1.

Verifying a hierarchy: Observe, the Roots strings assigned for a correct instance satisfy the following.

\section{The Roots strings (RS) conditions:}

- (RS0) The prefix of the Roots string at every node is in $\left[1,{ }^{*}\right]^{*}$ and its suffix is in $\left[0,{ }^{*}\right]^{*}$, (*because each node is a root of a level 0 fragment and continues being a root in its fragment until some level when it stops (if it does stop); when the node stops being a root, it never becomes a root again*)

- (RS1) the length of each Roots string is $\ell+1$, (*because there cannot be more than $\ell+1$ levels *) 


\begin{tabular}{|c|ccccc|}
\hline Roots & 0 & 1 & 2 & 3 & 4 \\
\hline $\mathrm{a}$ & 1 & 0 & 0 & 0 & 0 \\
$\mathrm{~b}$ & 1 & 1 & 0 & 0 & 0 \\
$\mathrm{c}$ & 1 & 0 & 0 & 0 & 0 \\
$\mathrm{~d}$ & 1 & $*$ & 0 & 0 & 0 \\
$\mathrm{e}$ & 1 & $*$ & 0 & 0 & 0 \\
$\mathrm{f}$ & 1 & 0 & 0 & 0 & 0 \\
$\mathrm{~g}$ & 1 & 1 & 1 & 1 & 0 \\
$\mathrm{~h}$ & 1 & $*$ & 1 & 0 & 0 \\
$\mathrm{i}$ & 1 & $*$ & 0 & 0 & 0 \\
$\mathrm{j}$ & 1 & 0 & 0 & 0 & 0 \\
$\mathrm{k}$ & 1 & 1 & 1 & 0 & 0 \\
$\mathrm{l}$ & 1 & 1 & 1 & 1 & 1 \\
$\mathrm{~m}$ & 1 & 1 & 0 & 0 & 0 \\
$\mathrm{n}$ & 1 & 0 & 0 & 0 & 0 \\
$\mathrm{o}$ & 1 & 0 & 0 & 0 & 0 \\
$\mathrm{p}$ & 1 & 1 & 0 & 0 & 0 \\
$\mathrm{q}$ & 1 & 0 & 0 & 0 & 0 \\
$\mathrm{r}$ & 1 & 0 & 0 & 0 & 0 \\
\hline
\end{tabular}

\begin{tabular}{|c|ccccc|}
\hline EndP & 0 & 1 & 2 & 3 & 4 \\
\hline $\mathrm{a}$ & up & none & none & none & none \\
$\mathrm{b}$ & down & up & none & none & none \\
$\mathrm{c}$ & up & none & none & none & none \\
$\mathrm{d}$ & up & $*$ & none & none & none \\
$\mathrm{e}$ & up & $*$ & none & none & none \\
$\mathrm{f}$ & up & down & none & none & none \\
$\mathrm{g}$ & down & none & down & up & none \\
$\mathrm{h}$ & down & $*$ & up & none & none \\
$\mathrm{i}$ & up & $*$ & none & none & none \\
$\mathrm{j}$ & up & none & none & none & none \\
$\mathrm{k}$ & down & down & up & none & none \\
$\mathrm{l}$ & down & down & down & down & none \\
$\mathrm{m}$ & down & up & none & none & none \\
$\mathrm{n}$ & up & none & none & none & none \\
$\mathrm{o}$ & up & none & none & none & none \\
$\mathrm{p}$ & down & up & none & none & none \\
$\mathrm{q}$ & up & none & none & none & none \\
$\mathrm{r}$ & up & none & none & none & none \\
\hline
\end{tabular}

\begin{tabular}{|c|ccccc|}
\hline Parents & 0 & 1 & 2 & 3 & 4 \\
\hline $\mathrm{a}$ & 1 & 0 & 0 & 0 & 0 \\
$\mathrm{~b}$ & 0 & 1 & 0 & 0 & 0 \\
$\mathrm{c}$ & 0 & 0 & 0 & 0 & 0 \\
$\mathrm{~d}$ & 1 & 0 & 0 & 0 & 0 \\
$\mathrm{e}$ & 0 & 0 & 0 & 0 & 0 \\
$\mathrm{f}$ & 1 & 0 & 0 & 0 & 0 \\
$\mathrm{~g}$ & 0 & 0 & 0 & 1 & 0 \\
$\mathrm{~h}$ & 0 & 0 & 1 & 0 & 0 \\
$\mathrm{i}$ & 0 & 0 & 0 & 0 & 0 \\
$\mathrm{j}$ & 1 & 0 & 0 & 0 & 0 \\
$\mathrm{k}$ & 0 & 0 & 1 & 0 & 0 \\
$\mathrm{l}$ & 0 & 0 & 0 & 0 & 0 \\
$\mathrm{~m}$ & 0 & 1 & 0 & 0 & 0 \\
$\mathrm{n}$ & 0 & 0 & 0 & 0 & 0 \\
$\mathrm{o}$ & 1 & 0 & 0 & 0 & 0 \\
$\mathrm{p}$ & 0 & 1 & 0 & 0 & 0 \\
$\mathrm{q}$ & 1 & 0 & 0 & 0 & 0 \\
$\mathrm{r}$ & 1 & 0 & 0 & 0 & 0 \\
\hline
\end{tabular}

\begin{tabular}{|c|ccccc|}
\hline Or-EndP & 0 & 1 & 2 & 3 & 4 \\
\hline $\mathrm{a}$ & 1 & 0 & 0 & 0 & 0 \\
$\mathrm{~b}$ & 1 & 1 & 0 & 0 & 0 \\
$\mathrm{c}$ & 1 & 0 & 0 & 0 & 0 \\
$\mathrm{~d}$ & 1 & 0 & 0 & 0 & 0 \\
$\mathrm{e}$ & 1 & 0 & 0 & 0 & 0 \\
$\mathrm{f}$ & 1 & 1 & 0 & 0 & 0 \\
$\mathrm{~g}$ & 1 & 1 & 1 & 1 & 0 \\
$\mathrm{~h}$ & 1 & 0 & 1 & 0 & 0 \\
$\mathrm{i}$ & 1 & 0 & 0 & 0 & 0 \\
$\mathrm{j}$ & 1 & 0 & 0 & 0 & 0 \\
$\mathrm{k}$ & 1 & 1 & 1 & 0 & 0 \\
$\mathrm{l}$ & 1 & 1 & 1 & 1 & 0 \\
$\mathrm{~m}$ & 1 & 1 & 0 & 0 & 0 \\
$\mathrm{n}$ & 1 & 0 & 0 & 0 & 0 \\
$\mathrm{o}$ & 1 & 0 & 0 & 0 & 0 \\
$\mathrm{p}$ & 1 & 1 & 0 & 0 & 0 \\
$\mathrm{q}$ & 1 & 0 & 0 & 0 & 0 \\
$\mathrm{r}$ & 1 & 0 & 0 & 0 & 0 \\
\hline
\end{tabular}

Table 2: Roots, EndP, Parents and Or-EndP for Figure1. 
- (RS2) the Roots string of the root $r$ of $T$ contains only "1"s and "*”s, and its $\ell$ 'th entry is " 1 ", (*because a zero in the $i$ th position would have meant that $r$ is not the root of its fragment of level $i$; the second part follows from the fact that the whole tree is a fragment of level $\ell$ and $r$ is its root *)

- (RS3) the first entry (at position 0 ) of every Root s string is " 1 ", (*because every node $v$ is the root of a singleton fragment of level 0 containing only node $v^{*}$ ),

- (RS4) the $\ell$ 'th entry of every non-root node is " 0 ", (*because only $r$ is the root of a fragment of level $\ell$, since that fragment is the whole tree *)

- (RS5) if the $j$ 'th entry of Roots $(v)$ is " 0 " for some node $v$ and $j \in[0, \ell]$, then the $j$ 'th entry of the Roots strings of $v$ 's parent in $T$ is not “*”.

(*because node $v$ belongs to a fragment $F$ of level $j$, but is not $F$ 's root; hence, $v$ 's parent belongs to $F$ of level $j$ too *)

It is easy to see that for any assignment of Roots strings $\mathcal{I}$ obeying rules RS1-RS5 there exists a unique hierarchy whose distributed representation is $\mathcal{I}$. Hence, we say that an assignment of Root s strings to the nodes of $T$ is legal if the strings obey the five Roots strings conditions above, namely RS1-RS5. For a given legal assignment of Roots strings $\mathcal{I}$, we refer to its induced hierarchy as the Roots-hierarchy of $\mathcal{I}$. Recall that at this point, we may assume that each node $v$ knows the value of $n$, and that each node knows whether it is the root of $T$. Hence, verifying that a given assignment of Roots strings is a legal one can be done locally, by letting each node look at its own string and the string of its parent only.

Identifying tree-neighbours in the same fragment: Obviously, for correct instances, the marker produces a legal assignment of Roots strings. For a general instance, if the verifier at some node finds that the assignment of Roots is not legal then it raises an alarm. Thus, (if no node raises an alarm), we may assume that hierarchy Roots-hierarchy exists, and that each node knows (by looking at its own label and the labels of its neighbours in the tree $T$ ), for every level $0 \leq j \leq \ell$,

1. whether it belongs to a fragment $F_{j}$ of level $j$, and if so:

2. which of its neighbours in $T$ belongs to $F_{j}$.

\subsection{Representing and verifying a candidate function for the Roots-hierarchy}

Having discussed the proof labeling for the hierarchy, we now describe the proof labeling scheme for the candidate function. Consider now a correct instance $G$ and the hierarchy $\mathcal{H}_{\mathcal{M}}$ produced by the marker algorithm. Recall, the candidate function $\chi_{\mathcal{M}}$ is given by the selected outgoing edges, which are precisely the minimum outgoing edges of the corresponding fragments, as identified by the construction algorithm SYNC_MST. We would like to represent this candidate function $\chi_{\mathcal{M}}$ distributively, and to verify that this representation indeed forms a candidate function. Moreover, we would make sure that each node $v$ be able to know, for each fragment $F$ containing it, whether it is an endpoint of the selected edge of $F$, and if so, which of its edges is the selected edge.

Representing a candidate function: Given a correct instance, and its corresponding legal assignment of Roots strings, we augment it by adding, for each node $v$, an additional string of $\ell+1$ entries named $\operatorname{EndP}(v)$. Intuitively, $\operatorname{EndP}(v)$ is used by the marker algorithm to mark the levels of the fragments for which $v$ is the endpoint of the minimum outgoing edge. Moreover, in a sense, $\operatorname{EndP}(v)$ also is a part of the marking of the specific edge of $v$ that is the minimum outgoing edge in that level (in the case that $v$ is indeed the endpoint). Let us now give the specific definition of that marking. 
Each entry in $\operatorname{EndP}(v)$ is one of four symbols, namely, "up", "down", "none" and "*”. The entries of $\operatorname{EndP}(v)$ are defined as follows. Fix an integer $j \in[0, \ell]$ and a node $v$. If $v$ does not belong to a fragment of level $j$ in $\mathcal{H}_{\mathcal{M}}$, then the $j$ 'th entry in $\operatorname{EndP}(v)$ is "**". Consider now $j \in[0, \ell]$ such that $v$ does belong to a fragment $F \in \mathcal{H}_{\mathcal{M}}$ of level $j$. If $v$ is not an endpoint of the candidate $\chi_{\mathcal{M}}(F)$ of $F$, then the $j$ 'th entry of $\operatorname{EndP}(v)$ is "none". Otherwise, node $v$ is an endpoint of $\chi_{\mathcal{M}}(F)$, i.e., $\chi_{\mathcal{M}}(F)=(v, u)$ (for some $u$ that is not in $F$ ). Consider two cases. If $u$ is $v$ 's parent in $T$ then the $j$ 'th entry of $\operatorname{EndP}(v)$ is set to "up". If, on the other hand, $u$ is a child of $v$ in $T$, then the $j$ 'th entry of $\operatorname{EndP}(v)$ is set to "down". See Table 1 for an example of EndP strings of nodes corresponding to Figure 1 .

Consider now a node $v$ that belongs to a level $j$ fragment $F \in \mathcal{H}_{\mathcal{M}}$. By inspecting its own label, node $v$ can find out whether it is an endpoint of a candidate of $F$ (recall, from the previous subsection, that it also knows whether or not it belongs to a level $j$ fragment). Moreover, in this case, we would like $v$ to actually be able to identify in one time unit, which of its incident (tree) edges is the candidate. Obviously, if the $j$ 'th entry in $\operatorname{EndP}(v)$ is "up", then the candidate $e$ is the edge leading from $v$ to its parent in $T$. Intuitively, in the case that the entry is "down", we would like to store this information in $v$ 's children to save space in $v$ (since $v$ may be the endpoint of minimum outgoing edges for several fragments, of several levels, and may not have enough space to represent all of them). Hence, we attach to each node $x$ another string called Parents $(x)$, composed also of $\ell+1$ bits. For $j \in[0, \ell]$, the $j$ 'th bit in $\operatorname{Parents}(x)$ is " 1 " if and only if $(y, x)$ is the candidate of the level $j$ fragment that contains $y$ (if one exists), where $y$ is the parent of $x$. See Table 1 for an example of Parents strings of nodes corresponding to Figure 1. Now, to identify $u$, node $v$ needs only to inspect the Parents strings of its children. In either of the above cases for the $\operatorname{EndP}(v)$ entry ("up" or "down"), we call $e$ the induced candidate of $F$.

Verifying a candidate function: Given a legal assignment of Roots strings, we say that assignments of EndP and Parents strings are legal if the following conditions hold:

- (EPS0) If the $j$ 'th entry of $\operatorname{Parents}(v)$ is " 1 " and $u$ is the parent of $v$, then the $j$ 'th entry of $\operatorname{EndP}(u)$ is "down",

(* because if $v$ indicates the minimum outgoing edge of $u$ 's fragment (of level $j$ ) leads from $u$ to $v$, then $v$ 's parent $u$ indicates this edge leads to one of $u$ 's children *)

- (EPS1) for each fragment $F$ of level $0 \leq j<\ell$ in the Root s-hierarchy, there exists precisely one node $v \in F$ whose $j$ 'th entry in $\operatorname{EndP}(v)$ is either "up" or "down",

(*because only one node $v$ in each fragment $F$ of level $j$ is the endpoint of the outgoing edge of $F *$ )

- (EPS2) for each node $v$, if the $j$ 'th entry in $\operatorname{EndP}(v)$ string is "down" then there exists precisely one child $u$ of $v$ such that the $j$ 'th entry in $\operatorname{Parent} \mathrm{s}(u)$ is " 1 ",

(*because the j'th entry in $\operatorname{EndP}(v)$ being "down" indicates its minimum outgoing edge leads to one of $v$ 's children (only one, since there is only one minimum outgoing edge of the fragment $F$ of level $j$ containing $v$ ); to remember which child, we mark this child $u$ by $\operatorname{Parents}(u)=1 *$ )

- (EPS3) for each node $v$, and for each $0 \leq j<\ell$, if the $j$ 'th entry in $\operatorname{EndP}(v)$ string is "up" then:

1. the $j$ 'th entry of $v$ 's Roots-string is " 1 ",

(*because node $v$ belongs to a different fragment $F_{v}$ of level $j$ than the level $j$ fragment of $v$ 's parent; hence, $v$ is the highest (closest to the root of the whole tree) in $F_{v}$, that is, $v$ is $F_{v}$ 's root *)

2. for every $i>j$, the $i$ 'th entry of $v$ 's Root s-string is not " 1 ", (*because fragment $F_{v}$ of $v$ in level $j$ merges with the fragment (of level $j$ ) of $v$ 's parent; hence, $v$ is not the highest in its fragments of levels $i>j^{*}$ )

- (EPS4) if the $j$ 'th entry in $\operatorname{Parent} s(v)$ is " 1 " then: 
1. the $j$ 'th entry of $v$ 's Roots-string is not " 0 ",

(*because node $v$ is not in the fragments of level $j$ of $v$ 's parent (see EPS2); hence, either $v$ is the root of its fragment of level $j$ (see EPS3, part 1), or $v$ does not belong to a fragment of level $j *$ )

2. for every $i>j$, the $i$ 'th entry of $v$ 's Roots-string is not " 1 ",

(*See EPS3 part $2 *)$

- (EPS5) for every non-root node $v$, there exists an index integer $j \in[0, \ell]$, such that either the $j$ 'th entry in Parent $\mathrm{s}(v)$ is 1 or the $j$ 'th entry in $\operatorname{EndP}(v)$ is "up".

(*because every node is the root of a fragment of level 0 ; at some level, $v$ 's fragment merges with the fragment of $v$ 's parent *)

Lemma 5.3 Consider a Roots-hierarchy $\mathcal{H}$ given by a legal assignment of Roots strings. The conditions EPS1-EPS5 above imply that legal assignments of EndP and Parents strings (with respect to $\mathcal{H}$ ) induce a candidate function $\chi: \mathcal{H} \backslash\{T\} \longrightarrow E(T)$.

Proof: Condition EPS1 implies that for each fragment $F \neq T$, there is precisely one node "suspected" as an endpoint of the induced candidate of $F$. Condition EPS2 together with the previous one, implies that there is precisely one induced candidate edge $\chi(F)$ for each fragment $F \neq T$. That is, these two conditions induce a function $\chi: \mathcal{H} \backslash\{T\} \longrightarrow E(T)$. Our goal now is to show that $\chi$ is, in fact, a candidate function. That is, we need to show that for every fragment $F \in \mathcal{H}$, we have $E(F)=\left\{\chi\left(F^{\prime}\right) \mid F^{\prime} \in \mathcal{H}(F)\right\}$. (Recall, $\mathcal{H}(F)$ denotes the set of fragments in $\mathcal{H}$ which are strictly contained in $F$.)

It follows by the second items in Conditions EPS3 and EPS4, that for every fragment $F \in \mathcal{H}$, we have

$$
E(F) \supseteq\left\{\chi\left(F^{\prime}\right) \mid F^{\prime} \in \mathcal{H}(F)\right\}
$$

In particular, we have $E(T) \supseteq\left\{\chi\left(F^{\prime}\right) \mid F^{\prime} \in \mathcal{H}(T)\right\}$. Now, by Condition EPS5, we get that each edge of $T$ is an induced candidate of some fragment. That is, we have:

$$
E(T)=\left\{\chi\left(F^{\prime}\right) \mid F^{\prime} \in \mathcal{H}(T)\right\}
$$

The first items in Conditions EPS3 and EPS4 imply that for every fragment $F \in \mathcal{H} \backslash\{T\}$, the edge $\chi(F)$ is outgoing from $F$. This fact, together with part (2) in the definition of a hierarchy, implies that for every fragment $F \in \mathcal{H}$,

$$
\left\{\chi\left(F^{\prime}\right) \mid F^{\prime} \notin \mathcal{H}(F)\right\} \bigcap E(F)=\emptyset .
$$

Equations (1), (2), and (3) imply that for every fragment $F \in \mathcal{H},\left\{\chi\left(F^{\prime}\right) \mid F^{\prime} \in \mathcal{H}(F)\right\}$. In other words, $\chi$ is a candidate function for $\mathcal{H}$, as desired.

Comment 5.1 Condition EPSO is not required in order to prove the above lemma. If the labels were assigned by our MST construction algorithm, condition EPSO holds too. Even though adding the condition seems redundant, we decided to add it because we believe it makes the reading more intuitive.

Now, to verify that assignments of EndP and Parents strings are legal with respect to a given legal assignment of Roots strings, we need to verify the five conditions above. Conditions EPS2-EPS5 can be verified easily, in 1 unit of time, while the first condition EPS1 needs additional information at each node to be verified in 1 unit of time. Specifically, verifying the rule amounts to verifying that exactly one of the nodes in a fragment of level $i$ has its $i$ 'th position in EndP equal to 1 . This is easy to do in a scheme that is very similar to Example NumK in Section 2.6. Hence, we omit this simple description (nevertheless, it is demonstrated in Table 2 in the example of the Or_EndP strings of nodes corresponding to Figure 1 . 


\subsection{The distributed marker algorithm}

A natural method for assigning the labels of the 1-proof labeling scheme described above (composed of the representation of $\mathcal{H}_{\mathcal{M}}$ and $\chi_{\mathcal{M}}$, and the strings Roots, Parents, EndP, and Or_EndP), is to follow the construction algorithm of the MST, namely SYNC_MST (see Section 4), which, in particular, constructs the hierarchy $\mathcal{H}_{\mathcal{M}}$ and the candidate function $\chi_{\mathcal{M}}$. Recall that the complexity of SYNC_MST is $O(n)$ time and $O(\log n)$ bits of memory per node.

Essentially, assigning the labels is performed by adding some set of actions to SYNC_MST. These actions do not change the values of any of the variables of the original algorithm. Also, we do not change the algorithm's flow of control, except for adding these actions. Since each action is just a new assignment to a new variable (of logarithmic size), the addition of these actions cannot violate the correctness of SYNC_MST, nor change its time and memory complexities (except by a constant factor). We note that adding these actions on top of SYNC_MST is not complicated, and can be realized using standard techniques. Hence, we omit it here. Hence, we obtain the following.

Lemma 5.4 There exists a distributed marker algorithm assigning the labels of the 1-proof labeling scheme described in Section 5 running in $O(n)$ time and using $O(\log n)$ bits of memory per node.

The lemma above together with Lemma 5.3 establishes Lemma 5.2 .

\section{Distributing pieces of information}

In the previous section, we described the verification that (1) a tree exists, (2) it is decomposed into a hierarchy of fragments, and (3) edges emanating from the fragments compose a candidate function (so that the tree is the collection of these edges). That verified the Well-Forming property. It is left to verify the Minimality property. That is, it is left to show that each edge of the candidate function is the minimum outgoing edge of some fragment in the hierarchy. The current section describes a part of the marker algorithm responsible for marking the nodes for this verification.

Informally, to perform the verification, each node must possess some information regarding each of the fragments $F$ containing it. The information regarding a fragment $F$ contains the weight of the selected edge of the fragment as well as the fragment identity, hence, it can be encoded using $O(\log n)$ bits. (The fragment identity is needed to identify the set $O_{F}$ of outgoing edges from $F$, and the weight of the selected edge is needed for comparing it to the weight of the other edges of $O_{F}$; this is how we detect that the selected edge is indeed the minimum). However, as mentioned, each node participates in $O(\log n)$ fragments, and hence, cannot hold at the same time all the information relevant for its fragments. Instead, we distribute this information among the nodes of the fragments, in a way that will allow us later to deliver this information efficiently to all nodes of the fragment. In this section, we show how to distribute the information regarding the fragments. In the next section, we explain how to exploit this distribution of information so that during the verification phase, relevant information can be delivered to nodes relatively fast and without violating the $O(\log n)$ memory size.

The piece of information $\operatorname{ID}(F)$ : As mentioned in Section 3.4, a crucial point in the scheme is letting each node $v$ know, for each of its incident edges $(v, u) \in E$ and for each level $j$, whether $u$ and $v$ share the same level $j$ fragment. (Note, in the particular case where $u$ is also a neighbour of $v$ in $T$, this information can be extracted by $v$ using $u$ 's data-structure, see Lemma 5.2.) Intuitively, this is needed in order to identify outgoing edges. For that purpose, we assign each fragment a unique identifier, and $v$ compares the identifier of its own level $j$ fragment 
with the identifier of $u$ 's level $j$ fragment. The identifier of a fragment $F$ is $\operatorname{ID}(F):=\operatorname{ID}(r(F)) \circ l e v(F)$, where $\operatorname{ID}(r(F))$ is the unique identity of the root $r(F)$ of $F$, and $\operatorname{lev}(F)$ is $F$ 's level. We also need each node $v$ to know the weight $\omega(F)$ of the minimum outgoing edge of each fragment $F$ containing $v$. To summarize, the piece of information $\mathrm{I}(F)$ required in each node $v$ per fragment $F$ containing $v$ is $\mathrm{I}(F):=\operatorname{ID}(F) \circ \omega(F)$. Thus, $\mathrm{I}(F)$ can be encoded using $O(\log n)$ bits.

At a very high level description, each node $v$ stores permanently $I(F)$ for a constant number of fragments $F$. Using that, $I(F)$ is "rotated" so that each node in $F$ "sees" $I(F)$ in $O(\log n)$ time. We term the mechanism that performs this rotation a train. A crucial point is having each node participate in only few trains. Indeed, one train passing a node could delay the other trains that "wish" to pass it. Furthermore, each train utilizes some (often more than constant) memory per node. Hence, many trains passing at a node would have violated the $O(\log n)$ memory constraint. In our solution, we let each node participate in two trains.

Let us recall briefly the motivation for two trains rather than one. As explained in Section 3.4.1, one way to involve only one train passing each node would have been to partition the nodes, such that each fragment would have intersected only one part of the partition. Then, one train could have passed carrying the pieces of information for all the nodes in the part. Unfortunately, we could not construct such a partition where the parts were small. A small size of each part is needed in order to ensure that a node sees all the pieces (the whole train) in a short time.

Hence, we construct two partitions of the tree. Each partition is composed of a collection of node-disjoint subtrees called parts. For each partition, the collection of parts covers all nodes. Hence, each node belongs to precisely two parts, one part per partition. For each part, we distribute the information regarding some of the fragments it intersects, so that each node holds at most a constant number of such pieces of information. Conversely, the information regarding a fragment is distributed to nodes of one of the two parts intersecting it. Furthermore, for any node $v$, the two parts corresponding to it encode together the information regarding all fragments containing $v$. Thus, to deliver all relevant information, it suffices to utilize one train per part (and hence, each node participates in two trains only). Furthermore, the partitions are made so that the diameter of each part is $O(\log n)$, which allows each train to pass in all nodes in short time, and hence to deliver the relevant information quickly. The mechanism of trains and their synchronization is described in the next section. The remaining of this current section is dedicated to the construction of the two partitions, and to explaining how the information regarding fragments is distributed over the parts of the two partitions.

\subsection{The two partitions}

Consider a correct instance, and fix the corresponding hierarchy tree $\mathcal{H}=\mathcal{H}_{\mathcal{M}}$. We now describe $t$ wo partitions of the nodes in $T$, called Top and Bottom. (The distributed algorithm that constructs the partitions is described later.) We also partition the fragments into two kinds, namely, top and bottom fragments.

Top and bottom fragments: Define the top fragments to be precisely those fragments whose number of nodes is at least $\log n$. Observe that the top fragments correspond to a subtree of the hierarchy tree $\mathcal{H}$. Name that subtree $T_{\text {Top }}$. All other fragments are called bottom. See the left side of Figure 2 for an illustration of the top fragments and the subtree $T_{\text {Top }}$.

\subsubsection{Partition Top}

Let us first describe partition Top. We first need to define three new types of fragments. 
Red, blue, and large fragments: A leaf fragment in subtree $T_{\text {Top }}$ is colored red. A fragment not in $T_{\text {Top }}$ which is a sibling in $\mathcal{H}$ of a fragment in $T_{\text {Top }}$ is colored blue. (Equivalently, a blue fragment is a fragment not in $T_{\text {Top }}$, whose parent fragment in $\mathcal{H}$ is a non-red fragment in $\left.T_{\mathrm{Top}}\right)$. The following observation is immediate.

Observation 6.1 The collection of red and blue fragments forms a partition $\mathcal{P}^{\prime}$ of the nodes of T. See Figure 2 for an illustration of partition $\mathcal{P}^{\prime}$.

To emphasize the fact that each non-blue child fragment of an internal fragment in $T_{\text {Top }}$ contains at least $\log n$ nodes, we call internal fragments in $T_{\text {Top }}$ large. Note, the large fragments are precisely the (strict) ancestors of the red fragments in $\mathcal{H}$. Since the ancestry relation in $\mathcal{H}$ corresponds to an inclusion relation between the corresponding (active) fragments in $T$, we obtain the following observation.

Observation 6.2 Each large fragment $F_{\text {large }}$ is composed of at least one red fragment $F_{\text {red }}$ as well as one or more blue ones, and does not contain any additional nodes (of course, the part may contain also the edges connecting those fragments).

Partition $\mathcal{P}^{\prime \prime}$ : Our goal now is to partition the nodes to parts such that each part contains precisely one red fragment and possibly several blue ones, and no additional nodes. Such a partition exists, since, it is just a coarsening of the partition $\mathcal{P}^{\prime}$ of the nodes to red and blue fragments. Moreover, the construction of some such a partition is trivial, following Observation 6.2 and the fact that the tree is a connected graph. The following procedure produces such a partition $\mathcal{P}^{\prime \prime}$ that has an additional property defined below. (A less formal description of the procedure is as follows: let pink parts be either red fragments, or the results of a merge between a red fragment and any number of blue ones. Now repeat the following as long as there are unmerged blue fragments: consider a blue fragment $F_{\text {blue }}$ who has a sibling pink fragment and, moreover touches that sibling; merge $F_{b l u e}$ with one of its sibling pink fragments it touches).

\section{Procedure Merge}

1. Initialize the set $\tilde{\mathcal{P}}$ of parts to include precisely the set of red parts. $(* \tilde{\mathcal{P}}$ is not yet a partition $*)$

2. Repeat while there are blue fragments not merged into parts of $\tilde{\mathcal{P}}$

(a) Let $F_{\text {large }}$ be a top fragment that contains a node $u$ that is not in any part of $\tilde{\mathcal{P}}$, where all the nodes of every child fragment of $F_{\text {large }}$ belong to parts of $\tilde{\mathcal{P}}$.

(b) Let $F_{\text {blue }}$ be the blue fragment containing $u$. (Note that we have $u \in F_{\text {blue }} \subset F_{\text {large }}$.) Let $\tilde{P} \in \tilde{\mathcal{P}}$ be some part that touches $F_{\text {blue }}$.

(c) Merge $F_{b l u e}$ with one such $\tilde{P}$. (This also removes $\tilde{P}$ from $\tilde{\mathcal{P}}$ and inserts, instead, the merged part $F_{\text {blue }} \cup \tilde{P}$

3. When the procedure terminates, $\mathcal{P}^{\prime \prime} \leftarrow \tilde{\mathcal{P}}$.

See Figure 2 for an illustration of partition $\mathcal{P}^{\prime \prime}$. It is easy to see (e.g., by induction on the order of merging in the above procedure) that partition $\mathcal{P}^{\prime \prime}$ is constructed in the following way: let $F_{\text {red }}$ be the red fragment in a part $\tilde{P}$. Then all the nodes in $\tilde{P}$ belong to ancestor fragments of $F_{\text {red }}$. This leads to the following observation.

Claim 6.3 Each part $P \in \mathcal{P}^{\prime \prime}$ intersects at most one level $j$ top fragment, for every $j$.

The property captured in the above claim is very useful. As can be seen later, this property means that the train in each part $\tilde{P}$ needs to carry only one piece of information for each level. 

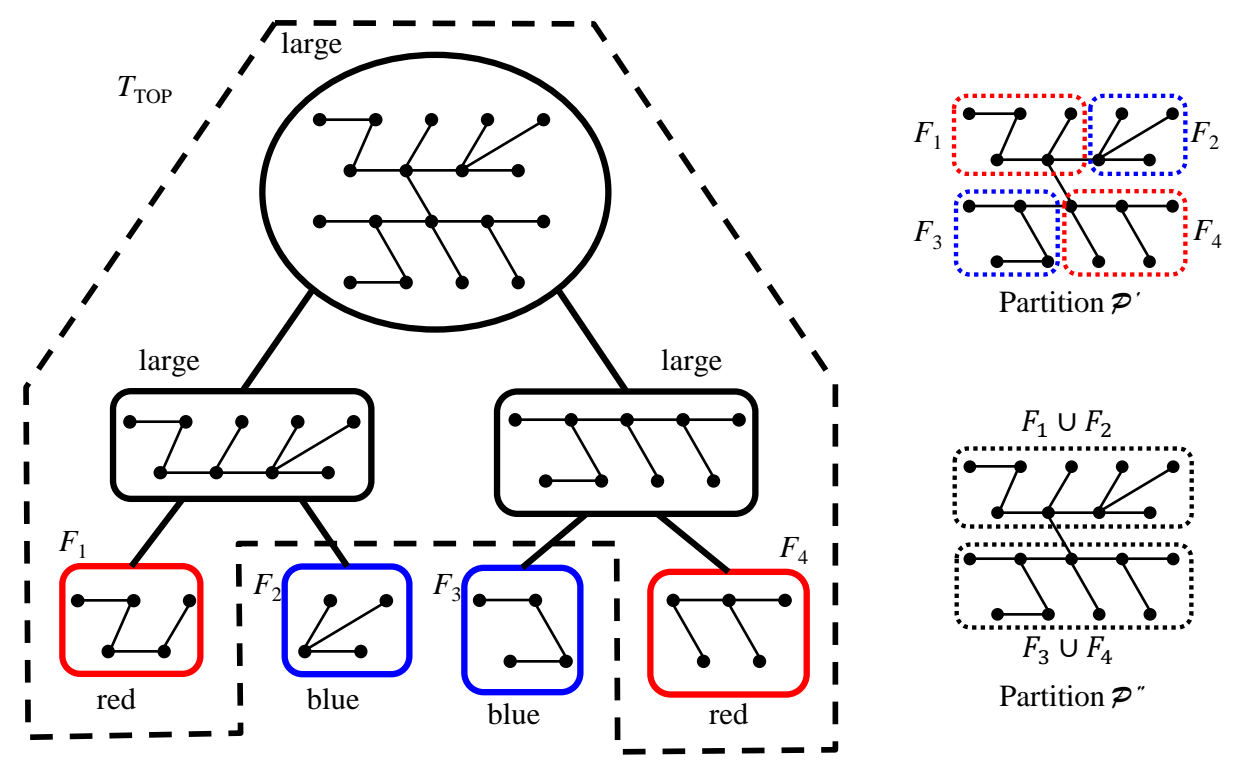

Figure 2: On the left: the top fragments and $T_{\text {Top }}$; On the right: partition $\mathcal{P}^{\prime}$ (above) and partition $\mathcal{P}^{\prime \prime}$ (below)

Partition Top: We would like to pass a train in each part $P$ of $\mathcal{P}^{\prime \prime}$. Unfortunately, the diameter of $P$ may be too large. In such a case, we partition $P$ further to neighbourhoods, such that each neighbourhood is a subtree of $T$ of size at least $\log n$ and of diameter $O(\log n)$. The resulted partition is called Top. The lemma below follows.

Lemma 6.4 For every part $P$ in partition Top, the following holds.

- $|P| \geq \log n$,

- $D(P)=O(\log n)$, where $D(P)$ is the diameter of $P$.

- $P$ intersects at most one level $j$ top fragment, for every $j$ (in particular, it intersects at most $\ell=\lceil\log n\rceil$ top fragments).

\subsubsection{Partition Bottom}

The bottom fragments are precisely those with less than $\log n$ nodes. The parts of the second partition Bottom are the following: (1) the blue fragments, and (2) the children fragments in $\mathcal{H}_{\mathcal{M}}$ of the red fragments. By Observation 6.1, this collection of fragments is a indeed a partition. Observe that each part of Bottom is a bottom fragment. Thus, the size, and hence the diameter, of each part $P$ of Bottom, is less than $\log n$. Figure 3 illustrates the bottom fragments and partition Bottom. Observe also that a part $P \in$ Bottom contains all of $P$ 's descendant fragments in $\mathcal{H}$ (recall, $P$ is a fragment, and the collection of fragments are a laminar family), and does not intersect other bottom fragments. Hence, we get the following.

Lemma 6.5 For every part $P$ of partition Bott om, the following holds:

- $|P|<\log n$, and

- $P$ intersects at most $2|P|<2 \log n$ bottom fragments. 


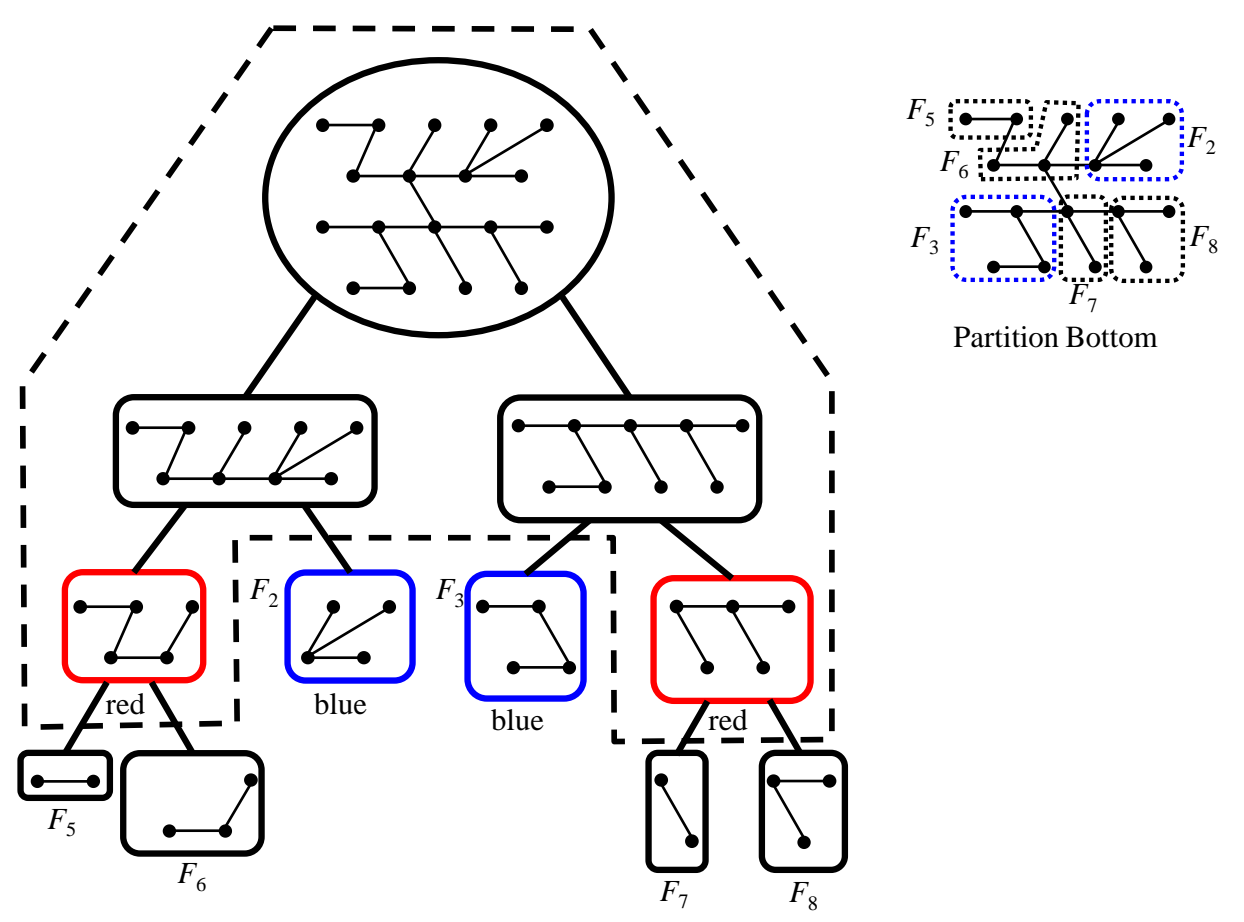

Figure 3: The bottom fragments and partition Bottom.

\subsubsection{Representations of the partitions}

In Section 6.3, we show that the above partitions Top and Bot tom can be constructed by a distributed algorithm that uses $O(\log n)$ memory and linear time. Each part $P$ of each of the two partitions is represented by encoding in a designated part of the label of each node in $P$, the identity $\operatorname{ID}(r(P))$ of the root of $P$ (the highest node of part $P)$. Recall that a node participates in only two parts (one of each partition), so this consumes $O(\log n)$ bits per node. Obviously, given this representation, the root of a part can identify itself as such by simply comparing the corresponding part of its label with its identity. In addition, by consulting the data-structure of a tree neighbour $u$, each node $v$ can detect whether $u$ and $v$ belong to the same part (in each of the two partitions).

A delicate and interesting point is that the verifier does not need to verify directly that the partitions Top or Bottom were constructed as explained here. This is explained in Section 8 .

\subsection{Distributing the information of fragments}

Fix a part $P$ of partition Top (respectively, Bottom). Recall that $P$ is a subtree of $T$ rooted at $r(P)$. Let $F_{1}, F_{2}, \cdots, F_{k}$ be the top (resp., bottom) fragments intersecting $P$, for some integer $k$. By Lemma 6.4 (resp., Lemma 6.5), we know that $k \leq \min \{2|P|, 2 \log n\}$. Assume w.l.o.g., that the indices are such that the level of $F_{i}$ is, at least, the level of $F_{i-1}$, for each $1<i \leq k$.

The information concerning part $P$ is defined as $I(P)=I\left(F_{1}\right) \circ I\left(F_{2}\right) \circ, \cdots, \circ I\left(F_{k}\right)$. We distribute this information over the nodes of $P$ as follows. We break $\mathrm{I}(P)$ into $|P|$ pairs of pieces. Specifically, for $i$ such that $1 \leq i \leq\lceil k / 2\rceil \leq|P|$, the $i$ 'th pair, termed $\mathrm{P} \mathrm{C}(i)$, contains $\mathrm{I}\left(F_{2 i-1}\right) \circ \mathrm{I}\left(F_{2 i}\right)$ (for odd $k, \mathrm{P} \mathrm{C}(\lceil k / 2\rceil)=\mathrm{I}\left(F_{k}\right)$ ).

The process of storing the pieces permanently at nodes of a part of the partition is referred to as the initialization of the trains. The distributed algorithm that implements the initialization of the trains using $O(\log n)$ memory 
size and linear time is described next. It is supposed to reach the same result of the following non-distributed algorithm (given just in order to define the result of the distributed one).

This non-distributed algorithm is simply the classical Depth First Search (DFS) plus the following operation in every node visited for the first time. Consider a DFS traversal over $P$ that starts at $r(P)$ and let df $\mathrm{S}(i)$ denote the the $i$ 'th node visited in this traversal. For each $i, 1 \leq i \leq\lceil k / 2\rceil$, dfs $(i)$ stores permanently the $i$ 'th pair of $\mathrm{I}(P)$, namely, $\mathrm{PC}(i)$.

\subsection{Distributed implementation}

Before describing the distributed construction of the two partitions, namely Top and Bottom, we need to describe a tool we use for efficiently executing several waves\&echoes operations in parallel. This Multi_Wave primitive (described below) performs a Wave\&Echo in every fragment in $\mathcal{H}$ of level $j$, for $j=0,1,2, \cdots, \ell$. Moreover, the $i+1$ th Wave\&Echo is supposed to start after the $i$ th Wave\&Echo terminates. Furthermore, all this is obtained in time $O(n)$.

\subsubsection{The Multi_Wave primitive}

We shall use this primitive only after the Roots string is already set, so that every node knows for each level, whether it is the root of a fragment of that level. Let us first present a slightly inefficient way to perform this. The root of the whole tree starts $\ell+1$ consecutive waves and echoes, each for the whole final tree. (By consecutive we mean that the $j+1$ th wave starts when the $j$ th wave terminates.) Let the level $j$ wave be called Wave ${ }^{I_{1}}(\mathrm{~T}, j)$ since it carries some instruction $I_{1}$, is sent over the whole tree $T$, and carries the information that it is meant for level $j$. A root $v_{j}$ of a fragment $F^{j}$ of level $j$, receiving Wave ${ }^{I_{1}}(\mathrm{~T}, j)$, then starts its own Wave\&Echo Wave ${ }^{I_{2}}\left(F^{j}, j\right)$ over its own fragment only. (Here, $I_{2}$ is some instruction possibly different than $I_{1}$.) A node who is not a root of a level $j$ fragment can echo $\operatorname{Wave}^{I_{1}}(\mathrm{~T}, j)$ as soon as all its children in the final tree (if it has any) echoed. A root $v_{j}$ echoes Wave ${ }^{I_{1}}(\mathrm{~T}, j)$ only after its own wave $\operatorname{Wave}^{I_{2}}\left(F^{j}, j\right)$ terminated (and, of course, after it also received the echoes of $\operatorname{Wave}^{I_{1}}(\mathrm{~T}, j)$ from all its children). The following observation follows immediately from the known semantics of Wave\&Echo.

Observation 6.6 Consider a fragment $F^{j}$ of level $j$ rooted at some $v_{j}$. The wave initiation by $v_{j}$ starts after all the waves involving its descendant fragments terminated (at the roots of the corresponding fragments).

The ideal time complexity of performing the above collection of $\ell$ waves is $\Theta(n \log n)$. In the case that the size of a level $j$ fragment $F^{j}$ is $2^{j} \leq\left|F^{j}\right|<2^{j+1}$, we can achieve the semantics of Observation 6.6 somewhat more time efficiently. The primitive that achieves this is termed a Multi_Wave. When invoking it, one needs to specify which instructions it carries. Informally, the idea is that the roots $R_{0}$ of level 0 fragments perform the wave (for level 0) in parallel, each in its own fragment of level 0 (a single node). Recall that a fragment $F_{1}$ of level 1 contains multiple fragments of level zero. The roots of these fragments of level zero report the termination of the level 0 wave to the root of $F_{1}$. Next, the roots $R_{1}$ of level 1 fragments perform the wave (for level 1 ) in parallel, each in its own fragment of level 1. The terminations are reported to level 2 fragment roots, etc., until the Multi_Wave terminates.

The Multi_Wave is started at the root of the final tree $T$ by a wave termed Multi_Wave $\left(T, I_{1}, I_{2}\right)$. Each node $v$ who receives Multi_Wave $\left(T, I_{1}, I_{2}\right)$ acts also as if $v$ has initiated a Wave $I_{2}\left(F^{0}, 0\right)$ on a tree containing only itself. Ensuring the termination and the semantics for level $\operatorname{Wave}^{I_{2}}\left(F^{0}, 0\right)$ is trivial. We now define the actions of levels higher than zero in an inductive manner. Every node $v$ who received (and forwarded to 
its children if it has any) Multi_Wave $\left(T, I_{1}, I_{2}\right)$, simulates the case that it received (and forwarded to its children) Wave ${ }^{I_{2}}\left(F^{j}, j\right)$ for every level $j$. However, $v$ is not free yet to echo $\operatorname{Wave}^{I_{2}}\left(F^{j}, j\right)$ until an additional condition holds as follows: When some wave $\operatorname{Wave}^{I_{2}}\left(F^{j}, j\right)$ terminates at the root $v_{j}$ of $F_{j}$, this root initiates an informing wave Wave ${ }^{\mathrm{Free}-I_{2}}\left(F^{j}, j\right)$ to notify the nodes in $F^{j}$ that the wave of level $j$ in their subtree terminated, and thus they are free to echo $\operatorname{Wave}^{I_{2}}\left(F^{j+1}, j+1\right)$. That is, a leaf of a $F^{j+1}$ fragment can echo Wave $^{I_{2}}\left(F^{j+1}, j+1\right)$ immediately when receiving Wave ${ }^{\mathrm{Free}-I_{2}}\left(F^{j}, j\right)$, and a non-leaf of Wave ${ }^{I_{2}}\left(F^{j+1}, j+1\right)$ may echo $\operatorname{Wave}^{I_{2}}\left(F^{j+1}, j+1\right)$ when it receives echoes from all its children in $F^{j+1}$.

Specifically, the convergecast is performed to the containing $j+1$ fragment as follows: a leaf of a level $j+1$ fragment who receives Wave ${ }^{\mathrm{Free}-I_{2}}\left(F^{j}, j\right)$ sends a message $\operatorname{Ready}\left(j+1, I_{2}\right)$ to its parent. A parent node sends message $\operatorname{Ready}\left(j+1, I_{2}\right)$ if it is not a root of a level $j+1$ fragment, and only after receiving $\operatorname{Ready}\left(j+1, I_{2}\right)$ from all its children. When a root of a level $j+1$ fragment receives the $\operatorname{Ready}\left(j+1, I_{2}\right)$ message from all of its children, it starts Wave ${ }^{\mathrm{Free}-I_{2}}\left(F^{j+1}, j+1\right)$. The Multi_Wave terminates at the root of the final tree when the wave for level $\ell$ terminates at that root. The informing wave $\operatorname{Wave}^{\mathrm{Free}-I_{2}}\left(F^{j}, j\right)$ itself needs no echo.

Observation 6.7 The efficient implementation of the multi-wave simulates the multiple waves analyzed in $\mathrm{Ob}$ servation 6.6. That is, it obtains the same result for the instructions $I_{1}$ and $I_{2}$ in every node.

Proof: Consider an alternative algorithm (for Multi_Wave) in which, when a root of fragment $F^{j+1}$ receives Multi_Wave $\left(T, I_{1}, I_{2}\right)$, it starts a wave Wave $-\operatorname{Ready}\left(j+1, I_{2}\right)$. Assume further, that the Ready $\left(j+1, I_{2}\right)$ messages are sent as echoes of Wave- $\operatorname{Ready}\left(j+1, I_{2}\right)$. Moreover, assume that an echo $\operatorname{Ready}\left(j+1, I_{2}\right)$ is sent by a node only after it received Wave ${ }^{\mathrm{Free}-I_{2}}\left(F^{j}, j\right)$. The claim for such an alternative algorithm would follow from Observation 6.6 and the known properties of Wave\&Echo. Now, it is easy to verify that the Multi_Wave described simulates that alternative algorithm. That is, (1) Multi_Wave $\left(T, I_{1}, I_{2}\right)$ is sent by a node $v_{j+1}$ who belongs to a fragment of level $j+1$ to its child $u$ in the same fragment exactly when it would have sent the imaginary Wave $-\operatorname{Ready}\left(j+1, I_{2}\right)$. This is easy to show by induction on the order of events. Moreover, at that time, the child $u$ knows the information carried by Wave - Ready $\left(j+1, I_{2}\right)$ since it knows (from its Roots) which fragments it shares with its parent (and for each one of them we simulate the case $u$ now receives Wave - Ready).

Observation 6.8 The ideal time complexity of performing a multi-wave on the hierarch $\mathcal{H}_{\mathcal{M}}$ is $O(n)$.

Proof: The wave started by the root consumes $O(n)$ time. Recall that hierarchy $\mathcal{H}_{\mathcal{M}}$ corresponds to active fragments during the construction of the MST by algorithm SYNC_MST. Hence, Lemma 4.1 implies that in hierarchy $\mathcal{H}_{\mathcal{M}}$, the size of a level $j$ fragment $F^{j}$ satisfies $2^{j} \leq\left|F^{j}\right|<2^{j+1}$. Thus, each wave started by a root of a fragment $F^{j}$ of level $j$ takes $O\left(2^{j}\right)$ time, and starts at time $O\left(2^{j}\right)$ after the initiation of the multi-wave.

\subsubsection{Distributed construction of partition $\mathcal{P}^{\prime}$}

The construction of partition $\mathcal{P}^{\prime}$ is performed in several stages. Each of the tasks below is performed using the Multi_Wave primitive. This is rather straightforward, given that the usage of Wave\&Echo as a primitive is very well studied. Below, we give some hints and overview.

First, we need to identify red fragments. It is easy to count the nodes in a fragment using Wave\&Echo to know which fragment has more than $\log n$ nodes. However, a large fragment that properly contains a red fragment is not red itself. Hence, the count is performed first in fragments lower in the hierarchy, and only then in fragments that are higher. Recall that the Multi_Wave primitive indeed completes first waves in fragments that are lower 
in the hierarchy, before moving to fragments that are higher. Hence, one execution of the Multi_Wave primitive allows to identify red fragment. At the end of this execution, the roots of fragments know whether they are the roots of red fragments or not. A similar technique can be applied to identify blue fragments.

A second task is to identify a large fragment $F_{\text {large }}$ that is not red, but has a child fragment who is red. It is an easy exercise to perform the construction using the Multi_Wave primitive.

The third task is that of identifying the blue fragments. A fourth task is to let each node in a blue fragment, and each node in a red fragment, know the color of their fragments. Again, designing these tasks is an easy exercise given the example of the first task above, and the Multi_Wave primitive.

\subsubsection{Constructing partition $\mathcal{P}^{\prime \prime}$}

It is rather straightforward to use waves\&echos to implement procedure Merge to generate partition $\mathcal{P}^{\prime \prime}$. The red fragments use waves\&echos to annex roots of sibling blue fragments. They become pink parts (in the terminology of paragraph 6.1.1. Then this is repeated in the parent fragment, etc. Since this process goes from a lower level fragment to higher and higher levels, the Multi_Wave primitive handles this well.

\subsubsection{Constructing partition Top}

Upon receiving the echoes for the Multi_Wave primitive constructing partition $\mathcal{P}^{\prime \prime}$, the root of the final tree instructs (by yet another Wave) each PART_LEADER of $\mathcal{P}^{\prime \prime}$ to start partitioning its part into parts of partition Top. That is, each part of $\mathcal{P}^{\prime \prime}$ is partitioned into subtrees, each of diameter $O(\log n)$ and of size $\Omega(\log n)$. This task is described in [57]. When it is completed, each part of Top is rooted at its highest node. Moreover, every node in that part is marked by the name of its part leader, in its variable called Top - Root. (Since Top is a partition, each node belongs only to one part; hence, this does not violate the $O(\log n)$ bits constraint.)

\subsubsection{Constructing partition Bottom}

Recall that the parts of the second partition Bottom are (1) the blue fragments and (2) the child fragments of red fragments. Let us term the latter green fragments. We already established that members and roots of blue fragments know that they are members and roots of blue fragments. The green fragments are notified in a similar way the blue ones were. That is, the root of the final tree starts a Wave\&Echo instructing the roots of the red fragments to notify child fragments that they are green.

Claim 6.9 The two partitions Top and Bottom described in Section 6.1 can be assigned in time $O(n)$ and memory size $O(\log n)$.

\subsubsection{Initializing the trains information}

First we describe a primitive that a root of a part $P$ uses for storing $I(F)$ of one given fragment $F \in P$. This is a well known distributed algorithm, so we do not describe it in detail. We use a distributed Depth First Search (DFS), see, e.g. [45, 22, 7]. Initially, all the nodes in a part $P$ are marked Vacant $(P)$. When the root of the part wants to store the $\mathrm{I}(F)$ of some fragment $F$, it sends this $\mathrm{I}(F)$ (with a token) to perform a DFS traversal of part $P$. The first time that token reaches a node marked Vacant $(P)$, it sets Vacant $(P)$ to false and stores $I(F)$ in that node. It is left to describe how the root of a part gets $I(F)$ for each $F$ whose $I(F)$ should reside in that part. 


\subsubsection{Storing $I$ in partition Top}

A part $P^{\prime \prime}$ in partition $\mathcal{P}^{\prime \prime}$ contains precisely one red fragment $F_{\text {red. }}$. Hence, we call such fragments red-centered. Consider a part $P$ in partition Top that was created from a red-centered part $P^{\prime \prime} \in \mathcal{P}^{\prime \prime}$. Recall that such a part $P$ should store only the I of top fragments it intersects. Since each such top fragment is an ancestor fragment of $F_{\text {red }}$, we let part $P$ store the I of all ancestor fragments of $F_{\text {red }}$. Hence, the set of I stored at $P$ includes the I of all fragments $P$ intersects, but may include more I's (of fragments intersecting $P^{\prime \prime}$ but not $P$ ). Nevertheless, note that, for every $j$, these other I's correspond to at most one fragment in level $j$. This follows simply from the fact that $F_{r e d}$ intersects at most one level $j$ fragment (see Claim 6.3). Recall also that the root of the part knows it is a root of a part (by comparing its Top - Root variable with its identity), and every node knows which part it belongs to (again, using its Top - Root variable) as well as who are its parent and children in the part. (The latter information a node can deduce by reading each tree neighbour.)

The root of the final tree $T$ starts a Multi_Wave over $T$. Fix a level $j$. The $j$ th wave of the multi-wave, which we term Send_Anc_Info $(T, j)$, signals the roots of every top fragment $F^{j}$ of level $j$ to obtain the information $I\left(F^{j}\right)$ and to send it to the roots of the parts of partition Top intersecting $F^{j}$. Consider a root $v_{j}$ of such a fragment $F^{j}$ who receives the signal of Send_Anc_In $f o(T, j)$. First, to obtain $I\left(F^{j}\right)$, node $v_{j}$ must find the weight of $e$, the minimum outgoing edge of $F^{j}$. Recall that the endpoint $u \in F_{j}$ of $e=(u, v)$ can identify it is the endpoint using the $j$ 'th position in $\mathrm{EndP}_{u}$, and can identify which of its incident edges is $e$. So, node $v_{j}$ starts another Wave\&Echo bringing the weight of $e$ to $v_{j}$. (Note that $v_{j}$ is the root of a single fragment in level $j$, though it may be the root of other fragments in other levels; hence, at the time of the wave of level $j$, it handles the piece of only one fragment, namely, $F_{j}$; hence, not congestion arises). When this wave terminates, $v_{j}$ sets $\mathrm{I}\left(F^{j}\right)=\left(\mathrm{ID}\left(v_{j}\right), \omega(e)\right)$ and starts another Wave\&Echo, called Anc_In $f \circ\left(F^{j}, j\right)$, conveying $\mathrm{I}\left(F^{j}\right)$ to roots of the parts of partition Top intersecting $F^{j}$. To implement this, $v_{j}$, the root of $F_{j}$, first broadcasts $I\left(F^{j}\right)$ to the nodes of $F^{j}$. At this point, each node in $F_{j}$ knows $\mathrm{I}\left(F^{j}\right)$. Next, our goal is to deliver $\mathrm{I}\left(F^{j}\right)$ to the roots of parts in partition Top intersecting $F^{j}$. However, note that since $F_{j}$ is a subtree, and all parts are subtrees, the roots of the parts of partition Top intersecting $F^{j}$ are all contained in $F_{j}$, except maybe the root $u_{j}$ of the part containing $v_{j}$. So, by now, all roots of parts in partition Top intersecting $F^{j}$, except maybe $u_{j}$, already know $\mathrm{I}\left(F^{j}\right)$. To inform $u_{j}$ it suffices to deliver $\mathrm{I}\left(F^{j}\right)$ up the tree, from $v_{j}$ to $u_{j}$. Since, all roots of parts in Top, and $u_{j}$ in particular, know they are roots, this procedure is trivial. Finally, to complete the wave at level $j$, a root of a Top part receiving $I\left(F_{j}\right)$ stores it in its part as described at top of this section (that is, storing each piece at a node in the part, following a DFS order).

Note, that since the diameter of a part in Top is of length $O(\log n)$, the wave of level $j$ can be implemented in $O\left(\left|F_{j}\right|+\log n\right)=O\left(2^{j}+\log n\right)$ time. Altogether, the Multi_Wave over $T$ is completed by time

$$
O\left(\sum_{j=1}^{\log n} 2^{j}+\log n\right)=O(n) .
$$

\subsubsection{Storing I in partition Bottom}

Recall that a part in partition Bottom is a fragment of size $O(\log n)$. The root of such a part $P$ collects the I of fragments in $P$ of each level $i$ by issuing a Wave\&Echo for level $i$. The weight of the minimum outgoing edge of each fragment $F^{i}$ of level $i$ is then collected by the root of $F^{i}$. This ensures that the $\mathrm{I}\left(F^{i}\right)$ for each fragment $F^{i}$ of level $i$ in the fragment arrives at $F^{i}$, s root. Finally, the Wave\&Echo collects the Is from the roots of the fragments in the Bottom part to the root of the part. It is easy to see the following.

Claim 6.10 The initialization of the trains information described in Section 6.3 can be done in time $O(n)$ and memory size $O(\log n)$. 
The next corollary that summarizes this section follows from Lemma 5.4 and Claims 6.9 and 6.10 .

Corollary 6.11 The marker algorithm $\mathcal{M}$ can be implemented using memory size $O(\log n)$ and $O(n)$ time.

\section{Viewing distributed information}

We now turn to the verifier algorithm of part of the proof labeling scheme that verifies the Minimality property.

Consider a node $v$ and a fragment $F_{j}(v)$ of level $j$ containing it. Recall that $\mathrm{I}\left(F_{j}(v)\right)$ should reside permanently in some node of a part $P$ to which $v$ belongs. This information should be compared at $v$ with the information $\mathrm{I}\left(F_{j}(u)\right)$ regarding a neighbour $u$ of $v$, hence both these pieces must somehow be "brought" to $v$. The process handling this task contains several components. The first is called a "train" and is responsible for moving the pieces' pairs $\mathrm{PC}_{\mathrm{C}}(i)$ through $P$ 's nodes, such that each node does not hold more than $O(\log n)$ bits at a time, and such that in short time, each node in $P$ "sees" all pieces, and in their correct order. (By short time, we mean $O(\log n)$ time in synchronous networks, and $O\left(\log ^{2} n\right)$ time asynchronous networks.)

Unfortunately, this is not enough, since $\mathrm{I}\left(F_{j}(v)\right)$ may arrive at $v$ at a different time than $\mathrm{I}\left(F_{j}(u)\right)$ arrives at $u$, hence some synchronization must be applied. Further difficulties arise from the fact that the neighbours of a node $v$ may belong to different parts, so different trains pass there. Note that $v$ may have many neighbours, and we would not want to synchronize so many trains.

A first idea to obtain synchronization would have been to utilize delays of trains. However, delaying trains at different nodes could accumulate, or could even cause deadlocks. Hence, we avoid delaying trains almost completely. Instead, each node $v$ repeatedly samples a piece from its train, and synchronizes the comparison of this piece with pieces sampled by its neighbours, while both trains advance without waiting. Perhaps not surprisingly, this synchronization turns out to be easier in synchronous networks, than in asynchronous ones.

This process presented below assumes that no fault occurs. The detection of faults is described later.

\subsection{The trains}

For simplicity, we split the task of a train into two subtasks, each performed repeatedly - the first, convergecast, moves (copies of) the pieces one at a time pipelined from their permanent locations to $r(P)$, the root of part $P$, according to the DFS order. (Recall, dfs $(i)$ stores permanently the $i$ 'th piece of $I(P)$.)

Definition 7.1 A cycle is a consecutive delivery of the $k$ pairs of pieces $P_{C}(1), P_{C}(2), \cdots, P_{C}(k)$ to $r(P)$.

Since we are concerned with at most $k \leq 2 \log n$ pairs of pieces, each cycle can be performed in $O(\log n)$ time. The second subtask, broadcast, broadcasts each piece from $r(P)$ to all other nodes in $P$ (pipelined). This subtask can be performed in $D(P)=O(\log n)$ time, where $D(P)$ is the diameter of $P$. We now describe these two subtasks (and their stabilization) in detail.

Consider a part $P$ (recall, a part is a subtree). The (pipelined) broadcast in $P$ is the simpler subtask. Each node contains a broadcast buffer for the current broadcast piece, and the node's children (in the part) copy the piece to their own broadcast buffer. When all these children of a node acknowledge the reception of the piece, the node can copy the next piece into its broadcast buffer. Obviously, this process guarantees that the broadcast of each piece is performed in $D(P)=O(\log n)$ time, where $D(P)$ is the diameter of $P$.

We now describe the convergecast subtask. Informally, this is a recursive process that is similar to a distributed DFS. The subtask starts at the root. Each node $v$ which has woken-up, first wakes-up its first child (that 
is, signals the first child to start). When the first child $u_{1}$ finishes (delivering to $v$ all the pieces of information in $u_{1}$ 's subtree), then $v$ wakes-up the next child, and so forth.

Each node holds two buffers of $O(\log n)$ bits each for two pieces of the train, besides its own piece (that it holds permanently). The node uses one of these buffers, called the incoming car, to read a piece from one of its children, while the other buffer, called the outgoing car is used to let its parent (if it has one) read the piece held by the node. A node $v \neq r(P)$ participates in the following simple procedure whenever signaled by its parent to wake-up. Let $u_{1}, u_{2}, \cdots, u_{d}$ denote the children of $v$ in $P$ (if any exists), ordered according to their corresponding port-numbers at $v$ (i.e., for $i<j$, child $u_{i}$ is visited before $u_{j}$ in the DFS tour).

Train Convergecast Protocol (performed at each node $v \neq r(P)$ )

(*Using two buffers: incoming car and outgoing car *)

1. Copy $v$ 's (permanent) piece into $v$ 's outgoing car

2. For $i=1$ to $d(* d$ is the number of $v$ 's children*)

(a) Signal $u_{i}$ to start performing the train algorithm; (*wake-up $\left.u_{i}{ }^{*}\right)$

(b) Repeat until $v$ receives a signal "finished" from $u_{i}$

i. Copy the piece from the outgoing car of $u_{i}$ to $v$ 's incoming car

ii. Wait until $v$ 's outgoing car is read by its parent (*to accomplish that, $v$ reads the incoming car of its parent and compares it with its outgoing car*)

iii. Move the piece from the incoming car to outgoing car (and, subsequently, empty the content of the incoming car);

3. Report "finished" to parent;

The train Convergecast protocol of the root $r(P)$ is slightly different. Instead of waiting for its parent to read each piece, it waits for the train Broadcast protocol (at the root) to read the piece to its own buffer. Instead of reporting "finished" to its parent, it generates a new start to its first child.

Theorem 7.1 Let $t_{0}$ be some time when the root $r=r(P)$ of $P$ initiated the "For" loop of the train Convergecast protocol. Each node in $P$ sees the pieces in the cycle $\left\{P_{C}(1), P_{C}(2), \cdots, P_{C}(\phi(P))\right\}$ in $O(\log n)$ time after $t_{0}$ in synchronous networks and in $O\left(\log ^{2} n\right)$ time after $t_{0}$ in asynchronous networks.

Proof: First observe that the train broadcast in a leaf node of the part who received a piece from its parent, does not need to pass that piece to any further children. Hence the train process does not incur a deadlock.

As mentioned before, once the root sees a piece, the broadcast protocol guarantees that this piece is delivered to all nodes in the part in $D(P)=O(\log n)$ time. Let $\tau^{\prime}$ denote the maximal time period between two consecutive times that the broadcast protocol at the root reads the buffer of the convergecast protocol to take a new piece (a piece is actually taken only if the convergecast has managed to bring there a new piece, after the broadcast process took the previous one). Now, denote $\tau=\max \left\{1, \tau^{\prime}\right\}$.

Observation 7.2 In synchronous networks, we have $\tau=1$. In asynchronous networks, we have $\tau \leq D(P)=$ $O(\log n)$.

The first part of the observation is immediate. To see why the second part of the observation holds, note that by the definition of time, it takes $O(D(P))$ for a chain of events that transfer a piece to a distance of $D(P)$, in the 
case that all the buffers on the way are free; note that there is no deadlock and no congestion for information flowing down the tree, away from the root; this can be seen easily by induction on the distance of a broadcast piece from the furthest leaf; clearly, if the distance is zero, the piece is consumed, so there is already a room for a new piece; the rest of the induction is also trivial.

We shall measure the time in phases, where each phase consists of $\tau$ time units. Let us start counting the time after time $t_{0}$, that is, we say that phase 0 starts at time $t_{0}$. Our goal now is to show that (for either synchronous or asynchronous networks), for each $1 \leq i<\phi(P)$, piece $\mathrm{PC}(i)$ arrives at the root within $O(\log n)$ phases.

We say that a node $v$ is holding a piece at a given time if either (1) $v$ keeps the piece permanently, or (2) at the given time, the piece resides in either $v$ 's incoming car or its outgoing car. Consider now phase $t$. For each $i$, where $1 \leq i \leq \phi(P)$, if the root $r$ held $\mathrm{PC}_{\mathrm{C}}(i)$ at some time between $t_{0}$ and the beginning of the phase $t$, then we say that $i$ is not t-relevant. Otherwise, $i$ is $t$-relevant. For any $t$-relevant $i$, where $1 \leq i \leq \phi(P)$, let $d_{t}(i)$ denote the smallest DFS number of a node $v$ holding $\mathrm{PC}(i)$ at the beginning of phase $t$. That is, $d_{t}(i)=\min \{\operatorname{dfs}(u) \mid u$ holds $\mathrm{PC}(i)$ at time $t\}$. For any $i$ that is not $t$-relevant, let $d_{t}(i)=0$. The following observation is immediate.

Observation 7.3 At any time $t$,

- For any $1 \leq i \leq \phi(P)$, we have $d_{t}(i) \geq d_{t+1}(i)$ (in other words, $d_{t}(i)$ cannot increase with the phase).

- For any $1 \leq i<\phi(P)$, we have $d_{t}(i) \leq d_{t}(i+1)$.

Informally, the following lemma gives a bound for the delay of a piece as a result of processing previous pieces.

Lemma 7.4 Let $x$ and $i$ be two integers such that $1 \leq i \leq x \leq \phi(P)$. Then, $d_{t}(x) \leq i-1$ for $t \geq 3 x-i$.

To prove the lemma, first observe that the condition holds for the equality case, that is, the case where $i=x$. Indeed, for each $1 \leq x \leq \phi(P)$, the node holding $\mathrm{PC}(x)$ permanently is at distance at most $x-1$ from the root. Hence, $d_{0}(x) \leq x-1$. Now, the condition follows since, by Observation 7.3 , $d_{t}(x)$ cannot increase with the phase.

We now prove the lemma using a double induction. The first induction is on $x$. The basis of the induction, i.e., the case $x=1$, is trivial, since it reduces to the equality case $i=x=1$.

Assume by induction that the condition holds for $x-1$ and any $i$, such that $1 \leq i \leq x-1 \leq \phi(P)$. We now prove that the condition holds for $x$ and any $1 \leq i \leq x$. This is done using a reverse induction on $i$.

The basis of this (second) induction, i.e., the case $i=x$, is an equality case and hence, it is already known to satisfy the desired condition. Now assume by induction, that the condition holds for $x$ and $i$, where $2 \leq i \leq x$, and let us show that it holds also for $x$ and $i-1$.

Let us first consider the case $i=2$. By the (first) induction hypothesis (applied with values $x-1$ and $i=1$ ), we know that

$$
d_{t^{\prime}}(x-1) \leq 0 \quad \text { where } \quad t^{\prime}=3 x-4 .
$$

Thus, at phase $t^{\prime}=3 x-4$, piece $\mathrm{P} \subset(x-1)$ is not $t^{\prime}$-relevant. That is, at that time, piece $\mathrm{P} \subset(x-1)$ is either in the outgoing car of the root $r(P)$ or in the root's incoming car. In the first case, the incoming car of the root is already empty at $t^{\prime}$. Otherwise, recall that, by definition, the broadcast process at the root consumes a piece from $r(P)$ 's outgoing car every phase (if there is a new piece there it has not taken yet). Hence, the outgoing car at 
$r(P)$ is consumed by phase $t^{\prime}+1$. By that phase, the root notices the piece is consumed, deliverers the content of its incoming car (namely, piece $\mathrm{PC}(x-1)$ ) to its outgoing car, and empties its incoming car.

On the other hand, by the second induction hypothesis, $d(x) \leq 1$ at the beginning of phase $3 x-2=t^{\prime}+2$. That is, $\mathrm{PC}(x)$ is at some child $v$ of the root. By the second part of Observation 7.3 , node $v$ is the child the root reads next, and, moreover no piece other than $\operatorname{PC}(x)$ is at the outgoing car of $v$. If at the beginning of phase $t^{\prime}+2$, piece $\mathrm{P} \mathrm{C}(x)$ is at the outgoing car of $v$, then the piece reaches the incoming car of the root already at phase $t^{\prime}+2$. Otherwise, by at most phase $t^{\prime}+3$, node $r(P)$ has a copy of $\mathrm{PC}(x)$ in its incoming car. This means that $d_{t^{\prime \prime}}(x) \leq 0$, where $t^{\prime \prime}=t^{\prime}+3 \leq 3 x-(i-1)$, as desired.

Now consider the case that $2<i$. By the second induction hypothesis, we have $d_{t^{\prime}}(x) \leq i-1$, where $t^{\prime}=3 x-i$. If $d_{t^{\prime}}(x) \leq i-2$ at the beginning of phase $t^{\prime}$ then we are done. Otherwise, let $v$ be the node holding $\mathrm{PC}(x)$ at the beginning of phase $t^{\prime}$ such that the distance (on the tree) of $v$ from $r$ is $i-1$. Let $u$ be $v$ 's parent. Our goal now is to show that $u$ holds $\operatorname{PC}(x)$ by phase $t^{\prime}+1$. The (first) induction hypothesis implies that the condition holds for the pair $x-1$ and $i-2$. That is,

$$
d_{t^{\prime}-1}(x-1) \leq i-3 .
$$

Thus, $\mathrm{P} \subset(x-1)$ has already been copied to $u$ 's parent $w$. The only reason $\mathrm{P} \subset(x-1)$ may be stuck at $u$ (perhaps at both the incoming and outgoing cars of $u$ ) at phase $t^{\prime}-1$, is that $u$ has not observed yet that its parent $w$ actually already copied $\mathrm{PC}(x-1)$. This is observed by $u$ by phase $t^{\prime}$ (when $u$ observes this, it empties the content of its incoming car). Thus, by phase $t^{\prime}+1$, node $u$ has a copy of $\mathrm{P} \subset(x)$, as desired. This concludes the proof of the lemma.

The theorem now follows from the lemma and from the fact that $\phi(P)=O(\log n)$.

Recognizing membership to arriving fragments: Consider now the case that a piece containing $I(F)$ carried by the broadcast wave arrives at some node $v$. Abusing notations, we refer to this event by saying that fragment $F$ arrives at $v$. Recall that $v$ does not have enough memory to remember the identifiers of all the fragments containing it. Thus, a mechanism for letting $v$ know whether the arriving fragment $F$ contains $v$ must be employed. Note that the level $j$ of $F$ can be extracted from $\mathrm{I}(F)$, and recall that it is already ensured that $v$ knows whether it is contained in some level $j$ fragment. Obviously, if $v$ is not contained in a level $j$ fragment then $v \notin F$. If $F_{j}(v)$ does exist, we now explain how to let $v$ know whether $F=F_{j}(v)$.

Consider first a train in a part $P \in$ Top. Here, $P$ intersects at most one level $j$ top fragment, for each level $j$ (see Lemma 6.4. Thus, this train carries at most one level $j$ fragment $F_{j}$. Hence, $F_{j}=F_{j}(v)$ if and only if $F_{j}(v)$ exists.

Now consider a train in a part $P \in$ Bottom. In this case, part $P$ may intersect several bottom fragments of the same level. To allow a node $v$ to detect whether a fragment $F_{j}$ arriving at $v$ corresponds to fragment $F_{j}(v)$, we slightly refine the above mentioned train broadcast mechanism as follows. During the broadcast wave, we attach a flag to each $\mathrm{I}(F)$, which can be either "on" or "off", where initially, the flag is "off". Recall that $\mathrm{I}(F)$ contains the identity $\operatorname{ID}(r(F))$ of the root $r(F)$ of $F$. When the broadcast wave reaches this root $r(F)$ (or, when it starts in $r(F)$ in the case that $r(F)=r(P)$ ), node $r(F)$ changes the flag to "on". In contrast, before transmitting the broadcast wave from a leaf $u$ of $F$ to $u$ 's children in $T$ (that do not belong to $F$ ), node $u$ sets the flag to "off". That way, a fragment $F$ arriving at a node $v$ contains $v$ if and only if the corresponding flag is set to "on". (Recall that the data structure lets each node know whether it is a leaf of a level $j$ fragment.) This process allows each node $v$ to detect whether $F=F_{j}(v)$.

To avoid delaying the train beyond a constant time, each node multiplexes the two trains passing via it. That is, it passes one piece of one train, then one piece of the other. 


\subsection{Sampling and synchronizing}

Fix a partition (either Top or Bottom), and a part $P$ of the partition. Node $u \in P$ maintains two variables: Ask $(u)$ and Show $(u)$, each for holding one piece $I(F)$. In Ask $(u)$, node $u$ keeps $I\left(F_{j}(u)\right)$ for some $j$, until $u$ compares the piece $\mathrm{I}\left(F_{j}(u)\right)$ with the piece $\mathrm{I}\left(F_{j}(v)\right)$, for each of its neighbours $v$. Let $\mathcal{E}(u, v, j)$ denote the event that node $u$ holds $\mathrm{I}\left(F_{j}(u)\right)$ in $\operatorname{Ask}(u)$ and sees $\mathrm{I}\left(F_{j}(v)\right)$ in Show $(v)$. (For simplicity of presentation, we consider here the case that both $u$ and $v$ do belong to some fragments of level $j$; otherwise, storing and comparing the information for a non-existing fragments is trivial.) For any point in time $t$, let $C(t)$ denote the minimal time interval $C(t)=[t, x(t)]$ in which every event of the type $\mathcal{E}(u, v, j)$ occurred. For the scheme to function, it is crucial that $C(t)$ exists for every time $t$. Moreover, to have a fast scheme, we must ensure that $\max _{t}|C(t)|$ is small.

Recall that the train (that corresponds to $P$ ) brings the pieces $I(F)$ in a cyclic order. When $u$ has done comparing $\mathrm{I}\left(F_{j}(u)\right)$ with $\mathrm{I}\left(F_{j}(v)\right)$ for each of its neighbours $v$, node $u$ waits until it receives (by the train) the first piece $\mathrm{I}(F)$ following $\mathrm{I}\left(F_{j}(u)\right)$ in the cyclic order, such that $F$ contains $u$ (recall that $u$ can identify this $F$ ). Let us denote the level of this next fragment $F$ by $j^{\prime}$, i.e., $F=F_{j^{\prime}}(u)$. Node $u$ then removes $I\left(F_{j}(u)\right)$ from $\operatorname{Ask}(u)$ and stores $\mathrm{I}\left(F_{j^{\prime}}(u)\right)$ there instead, and so forth. Each node $u$ also stores some piece $\mathrm{I}\left(F_{i}(u)\right)$ at Show $(u)$ to be seen by its neighbours. (Note that the value at Show $(u)$ may be different than the one at Ask $(u)$.)

Let us explain the comparing mechanism. Assume that everything functions correctly. In particular, assume that the partitions and the distribution of the information are as described above, and the trains function correctly as well. Let us first focus our attention on the simpler and seemingly more efficient synchronous case.

\subsubsection{The comparing mechanism in synchronous networks}

Fix a node $v$. In a synchronous network, node $v$ sees $\operatorname{Show}(u)$ in every pulse, for each neighbour $u$. Let every node $u$ store in Show $(u)$ each piece that arrives in the train (each time, replacing the previous content of Show $(u)$ ). Hence, by Theorem 7.1. given a level $j$, node $v$ sees $I\left(F_{j}(u)\right)$ (if such exists) within $O(\log n)$ time. Put differently, if $v$ waits some $O(\log n)$ time (while $\mathrm{I}\left(F_{j}(v)\right)$ is in $\operatorname{Ask}(v)$ ), node $v$ sees $\mathrm{I}\left(F_{j}(u)\right)$ in $\operatorname{Show}(u)$ for each neighbour $u$. (We do not assume that $u$ keeps track of which neighbours $v$ has already seen $\mathrm{I}\left(F_{j}(u)\right.$ ); node $v$ simply waits sufficient time - to allow one cycle of the train, while looking at its neighbours, looking for their I for level $j$.) Subsequently, node $v$ waits another $O(\log n)$ rounds until the train brings it $I\left(F_{j^{\prime}}(v)\right)$ and stores it in $\operatorname{Ask}(v)$, and so forth. In other words, we have just established that event $\mathcal{E}\left(v, u, j^{\prime}\right)$ occurs within $O(\log n)$ time after $v$ stores $I\left(F_{j^{\prime}}(v)\right)$ in $\operatorname{Ask}(v)$, which happens $O(\log n)$ time after event $\mathcal{E}(v, u, j)$, and so forth. The time for at most $\log n+1$ such events to occur (one per level $j$ ) is $O\left(\log ^{2} n\right.$ ).

Lemma 7.5 In a synchronous environment, for each node $v$ and its neighbour $u$, all events of type $\mathcal{E}(v, u, j)$ (for all levels $j$ ) occur within time $O\left(\log ^{2} n\right)$.

\subsubsection{The comparing mechanism in asynchronous networks}

In an asynchronous network, without some additional kind of a handshake, node $u$ cannot be sure that the piece in $\operatorname{Show}(u)$ was actually seen by its neighbours. (Intuitively, this is needed, so that $u$ can replace the piece with the next one.) Moreover, it is not easy to perform such handshakes with all of $u$ 's neighbours, since $u$ does not have enough memory to keep track on which of its neighbours $v$ has seen the piece and which has not yet. First, let us describe a simple, but somewhat inefficient handshake solution. A more efficient one is presented later.

Each node $v$, holding some piece $\mathrm{I}\left(F_{j}(v)\right)$ in $\operatorname{Ask}(v)$, selects a neighbour $u$ and acts as a "client": that is, node $v$ writes in its register Want the pair $(\operatorname{ID}(u), j)$. Node $v$ then looks repeatedly at Show $(u)$ until it sees 
$\mathrm{I}\left(F_{j}(u)\right)$ there. At the same time, each node $u$ also has a second role - that of a "server". That is, each node rotates these two roles: it performs one atomic action as a server and one as a client. Acting as a server, $u$ selects a client to serve (in a round robin order). If the client has written some $(\operatorname{ID}(w), i)$ in the client's Want, for $w \neq u$, then $u$ chooses another client. On the other hand, if the client wrote $(\operatorname{ID}(u), j)$ in the client's Want, then $u$ waits until it receives by the train $\mathrm{I}\left(F_{j}(u)\right)$ and stores it in $\operatorname{Show}(u)$. A trivial handshake then suffices for $u$ to know that this value has been read by the client. Node $u$, in its role as a server, can then move to serve its next neighbour, and node $v$, in its role as a client, can move on to the next server. In particular, if the client $v$ has already received service from all its neighbours for $\mathrm{I}\left(F_{j}(v)\right)$, then $v$ waits until the train brings it the next piece $\mathrm{I}\left(F_{j^{\prime}}(v)\right)$ that $v$ needs to compare.

Consider now the time a client $v$ waits to see $\mathrm{I}\left(F_{j}(u)\right)$ for one of its neighbours $u$. Before serving $v$, the server $u$ may serve $O(\Delta)$ neighbours. By Theorem 7.1 (applied for the asynchronous setting), each service takes $O\left(\log ^{2} n\right)$ time. In addition, the client needs services from $\Delta$ servers, and for $O(\log n)$ values of $j$. The total time for all the required events to happen in this simple handshake mechanism is, thus, $O\left(\Delta^{2} \log ^{3} n\right)$.

Let us now describe the more efficient asynchronous comparison mechanism that requires only $O\left(\Delta \log ^{3} n\right)$ time. Before dwelling into the details of the comparison mechanism, let us first describe a difference in the way we employ the train. Recall that in the simple solution above (as well as in the synchronous case), the movement of trains was independent from the actions of the comparison mechanism, and hence, by Theorem 7.1, each train finishes a cycle in $O\left(\log ^{2} n\right)$ time. In contrast, a train here may be delayed by the nodes it passes, in a way to be described. Crucially, as we show later, the delay at each node is at most some constant time $c$, and hence, the time a train finishes a cycle remains asymptotically the same, namely, $O\left(\log ^{2} n\right)$.

As before, node $v$, holding $\mathrm{I}\left(F_{j}(v)\right)$ in Ask $(v)$ chooses a server $u$ among $v$ 's neighbours and reads Show $(u)$. Another small, but crucial addition to the actions taken in the simple procedure, is the following: if, when reading Show $(u)$, node $v$ reads $\mathrm{I}\left(F_{j}(u)\right)$, then $\mathcal{E}(v, u, j)$ occurred, and $v$ moves on to read another neighbour. This is illustrated in Figures 4 and 5 .

Only in the case that $\mathrm{I}\left(F_{j}(u)\right)$ is not at $\operatorname{Show}(u)$ at that time (see Figure 6, node $v$ sets Want $(v) \leftarrow$ $(\operatorname{ID}(u), j)$ (see Figure 7). In this case, we say that $v$ files a request for $j$ at $u$. This request stays filed until the value of $\operatorname{Show}(u)$ is the desired one and $\mathcal{E}(v, u, j)$ occurs. Similarly to the synchronized setting, in the case that $v$ has just finished seeing $\mathrm{I}\left(F_{j}(u)\right)$ in every neighbour $u$, node $v$ first waits until it gets by the train, the next piece $\mathrm{I}\left(F_{j^{\prime}}(v)\right)$ in the cycle, and then puts $\mathrm{I}\left(F_{j^{\prime}}(v)\right)$ as the new content of Ask $(v)$.

Now consider any node $u$ in its role as a server. It reads all the clients. (Recall that the ideal time complexity assumes this can be performed in one time unit.) When node $u$ receives $\mathrm{I}\left(F_{j}(u)\right)$ from the train, it puts this value in $\operatorname{Show}(u)$. It now delays the train as long as it sees any client $v$ whose $\operatorname{Want}(v)=(\operatorname{ID}(u), j)$. In particular, node $u$ keeps $\mathrm{I}\left(F_{j}(u)\right)$ in Show $(u)$ during this delay time period. If $u$ has not read any neighbour $v$ such that Want $(v)=(\operatorname{ID}(u), j)$, then $u$ stops delaying the train, waits for receiving the next piece $\mathrm{I}\left(F_{j^{\prime}}(u)\right)$ from the train, and uses it to replace the content of $\operatorname{Show}(u)$.

We define the Ask cycle of a node $v$. This is the time interval starting at the time a client $v$ replaces the content of $\operatorname{Ask}(v)$ from $\mathrm{I}\left(F_{j \max }(v)\right)$ to $\mathrm{I}\left(F_{j^{\min }}(v)\right)$, and until (and excluding) the time $v$ does that again. Here, $j^{\max }$ is the highest level of a piece in that train, such that $F_{j \max }(v)$ exists, and $j^{\text {min }}$ is the smallest level of a piece in that train, such that $F_{j \min }(v)$ exists.

Lemma 7.6 The total length of a Ask cycle of a node $v$ is $O\left(\Delta \log ^{3} n\right)$.

Proof: Fix a node $u$ and let $t_{u}^{1}$ be some time that $u$ starts storing $\mathrm{I}\left(F_{j}(u)\right)$ in $\operatorname{Show}(u)$, for some level $j$; moreover, $\mathrm{I}\left(F_{j}(u)\right)$ is stored there until some time $t_{u}^{2}$ when $u$ replaces the content of Show $(u)$ again.

Recall, node $u$ delays the train and keeps $I\left(F_{j}(u)\right)$ in $\operatorname{Show}(u)$ as long as it sees any client $v$ such that 

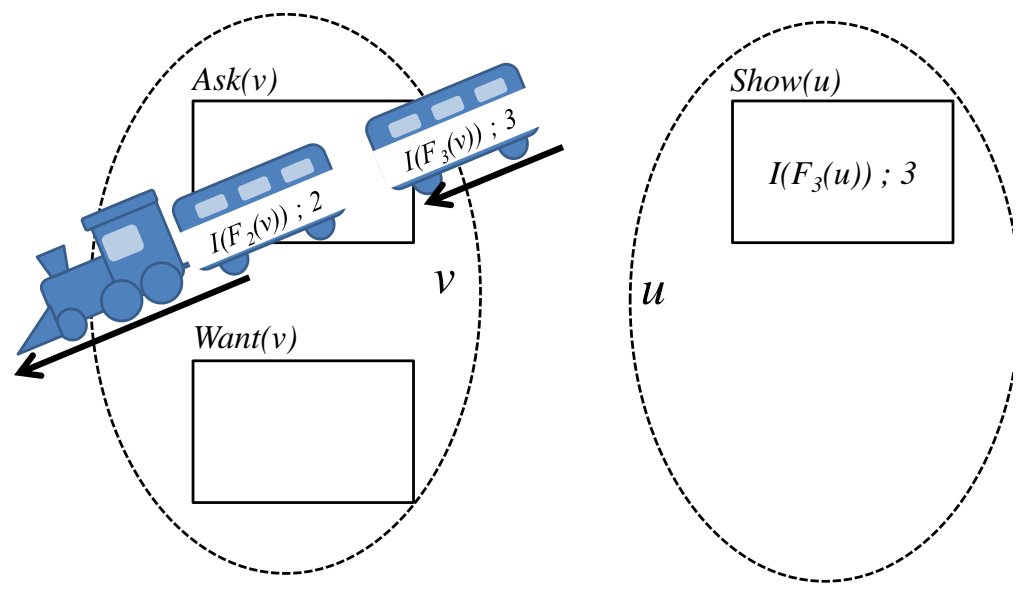

Figure 4: Node $v$ receives the next piece (for $j=3$ ) to compare.

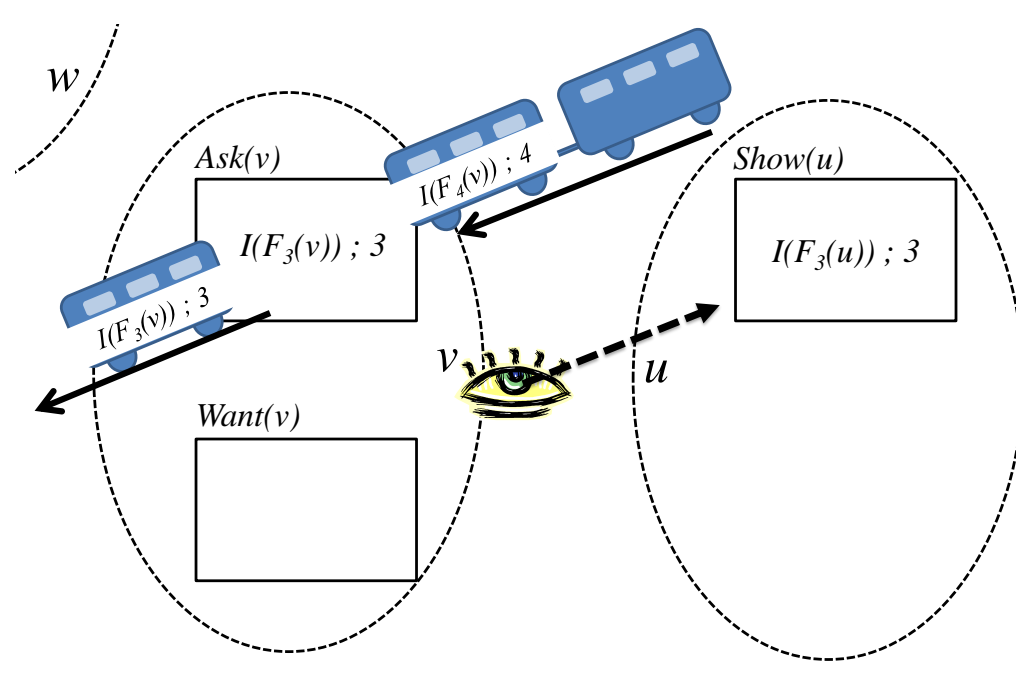

Figure 5: First case, $\mathcal{E}(v, u, 3)$ occurred the first time $v$ reads $I\left(F_{3}(u)\right)$. Next, $v$ may look at its next node, $w$. 


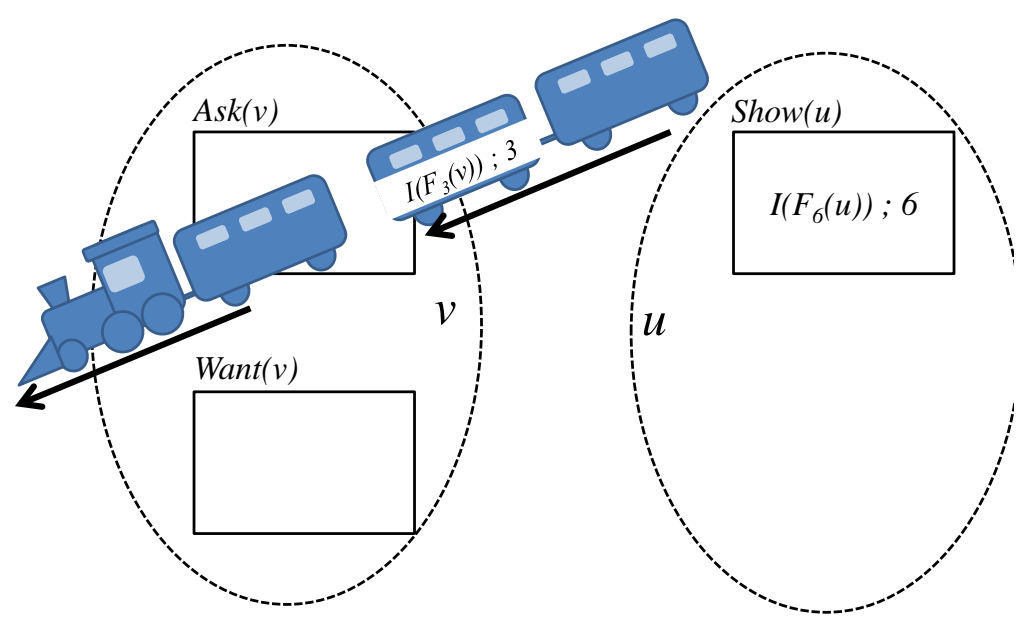

Figure 6: Second case, $\mathcal{E}(v, u, 3)$ does not occur immediately.

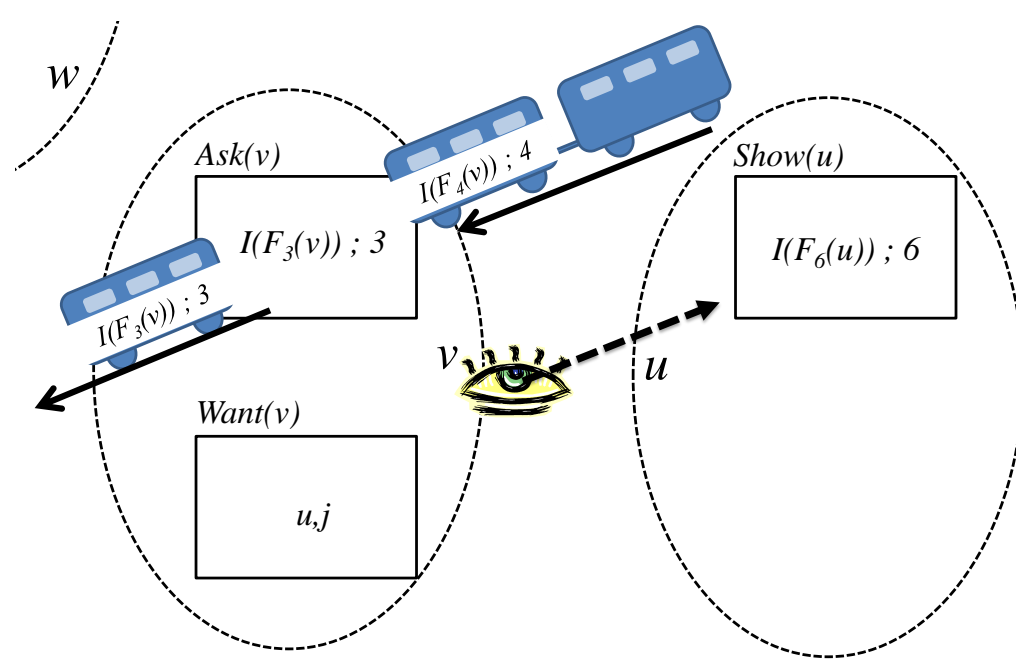

Figure 7: Node $v$ files a request at $u$ for $j=3$. 


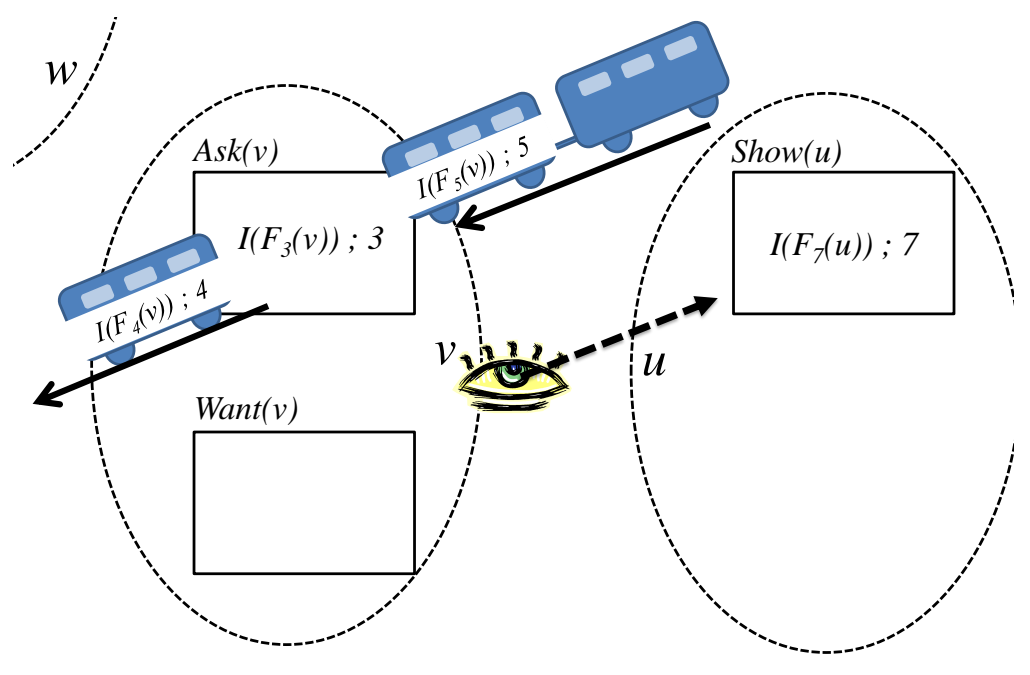

Figure 8: The trains at $v$ and at $u$ do not stop.

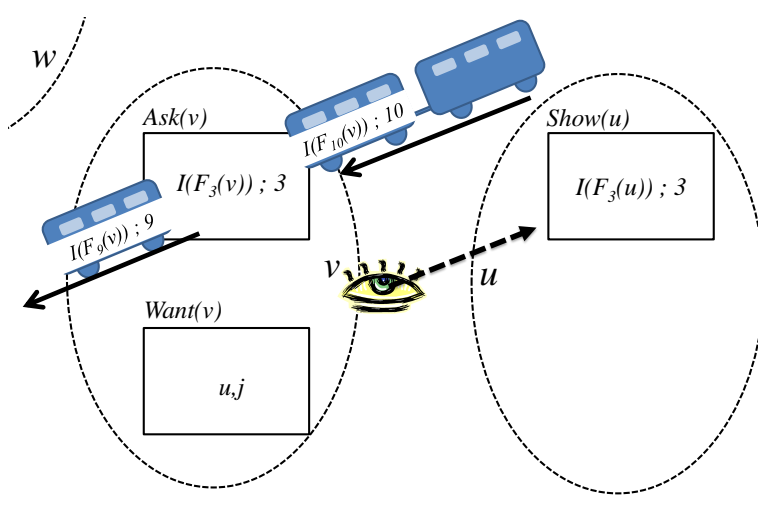

Figure 9: The server $u$ received the requested piece at last. 
Want $(v)=(\operatorname{ID}(u), j)$; when it sees that no such neighbour $v$ exists, it stops delaying the train and waits for the train to deliver it the next piece $I\left(F_{j^{\prime}}(u)\right)$ to be used for replacing the content of $\operatorname{Show}(u)$. We now claim that the delay time period at node $u$ is at most some constant time. To prove that, we first show that there exists a constant $c$ such that no client $v$ has $\operatorname{Want}(v)=(\operatorname{ID}(u), j)$ in the time interval $\left[t_{u}^{1}+c, t_{u}^{2}\right]$. Indeed, for each neighbour $v$ of $u$, let $t_{v}$ be the first time after $t_{u}^{1}$ that $v$ reads the value of Show $(u)$. Clearly, there exists a constant $c$ (independent of $u$ and $v$ ) such that $t_{v} \in\left[t_{u}^{1}, t_{u}^{1}+c\right]$. Right at time $t_{v}$, the content of Want $(v)$ stops being $(\operatorname{ID}(u), j)$ (if it were before), since $\mathrm{I}\left(F_{j}(u)\right)$ is the value of $\operatorname{Show}(u)$ during the whole time interval $\left[t_{u}^{1}, t_{u}^{2}\right]$. Moreover, during the time interval $\left[t_{v}, t_{u}^{2}\right]$, node $v$ does not file a request for $j$ at $u$, since again, whenever it reads Show $(u)$ during that time interval, it sees $\mathrm{I}\left(F_{j}(u)\right)$. Hence, no client $v$ has $\operatorname{Want}(v)=(\operatorname{ID}(u), j)$ in the time interval $\left[t_{u}^{1}+c, t_{u}^{2}\right]$. Now, from time $t_{u}^{1}+c$, it takes at most some constant time to let $u$ observe that none of its neighbours $v$ has $\operatorname{Want}(v)=(\operatorname{ID}(u), j)$. This establishes the fact that the delay of the train at each node is at most some constant. Hence, as mentioned before, the time the train finishes a cycle is $O\left(\log ^{2} n\right)$. (It is also easy to get convinces that this delay does not prevent the train from being self-stabilizing.)

Next, consider the time that some node $v$ starts holding $\mathrm{I}\left(F_{j}(v)\right)$ in Ask $(v)$. Consider a neighbour $u$ of $v$. The time it takes for $v$ until it sees $\mathrm{I}\left(F_{j}(u)\right)$ in Show $(u)$ is $O\left(\log ^{2} n\right)$. Hence, a client $v$ waits $O\left(\log ^{2} n\right)$ for each request $v$ files at a server $u$ for a value $j$. The total time that $v$ waits for a service of $j$ at all the servers is then $O\left(\Delta \log ^{2} n\right)$. From that time, $v$ needs to wait additional $O\left(\log ^{2} n\right)$ time to receive from the train the next piece $\mathrm{I}\left(F_{j^{\prime}}(v)\right)$ (to replace the content of Ask $(v)$ ). Summing this over the $O(\log n)$ pieces in the cycle, we conclude that the total time of an Ask cycle of $v$ is $O\left(\Delta \log ^{3} n\right)$.

Lemma 7.7 If (1) two partitions are indeed represented, such that each part of each partition is of diameter $O(\log n)$, and the number of pieces in a part is $O(\log n)$, and (2) the trains operate correctly, then the following holds.

- In a synchronous network, $\max _{t}|C(t)|=O\left(\log ^{2} n\right)$.

- In an asynchronous network, $\max _{t}|C(t)|=O\left(\Delta \log ^{3} n\right)$.

\section{Local verifications}

In this section, we describe the measures taken in order to make the verifier self-stabilizing. That is, the train processes, the partitions, and also, the pieces of information carried by the train may be corrupted by an adversary. To stress this point and avoid confusion, a piece of information of the form $z \circ j \circ \omega$, carried by a train, is termed the claimed information $\hat{I}(F)$ of a fragment $F$ whose root ID is $z$, whose level is $j$, and whose minimum outgoing edge is $\omega$. Note that such a fragment $F$ may not even exist, if the information is corrupted. Conversely, the adversary may also erase some (or even all) of such pieces corresponding to existing fragments. Finally, even correct pieces that correspond to existing fragments may not arrive at a node in the case that the adversary corrupted the partitions or the train mechanism. Below we explain how the verifier does detect such undesirable phenomena, if they occur. Note that for a verifier, the ability to detect with assuming any initial configuration means that the verifier is self-stabilizing, since the sole purpose of the verifier is to detect. We show, in this section, that if an MST is not represented in the network, this is detected. Since the detection time (the stabilization time of the verifier) is sublinear, we still consider this detection as local, though some of the locality was traded for improving the memory size when compared with the results of [54, 55].

Verifying that some two partitions exist is easy. It is sufficient to (1) let each node verify that its label contains the two bits corresponding to the two partitions; and (2) to have the root $r(T)$ of the tree verify that the value of each of its own two bits is 1. (Observe that if these two conditions hold then (1) $r(T)$ is a root of one part in each 
of the two partitions; and (2) for a node $v \neq r(T)$, if one of these two bits in $v$ is zero, then $v$ belongs to the same part in the corresponding partition as its parent.) Note that this module of the algorithm self-stabilizes trivially in zero time.

It seems difficult to verify that the given partitions are as described in Section 6.1, rather than being two arbitrary partitions generated by an adversary. Fortunately, this verification turns out to be unnecessary. (As we shall see, if the components at the nodes do not describe an MST, no adversarial partitioning can cause the verifier to accept this as representing an MST; if partitions are represented, we just need to verify that a part is not too large for the time complexity).

First, for the given partitions, it is a known art to self-stabilize the train process. That is, the broadcast part of the train is a standard flooding, for which the self stabilization has been heavily studied, see, in particular, [20, 24]. For the convergecast, first, note that pieces are sent up the tree. Hence, they cannot cycle, and cannot get "stuck". Moreover, it is easy to get convinced that only pieces that are already in some buffer (either incomming, or outgoing, or permanent) can be sent. Finally, notice that the order of the starting of the nodes is exactly the DFS order. The stabilization of the DFS process is well understood [23]. It is actually easier here, since this is performed on a tree (recall that another part of the verifier verifies that there are no cycles in the tree).

Finally, composing such self-stabilizing primitives in a self-stabilizing manner is also a known art, see e.g. [34, 49, 64, 52]. In our context, once the DFS part stabilizes, it is easy to see the pieces flow up the tree stabilizes too. This leads to the following observation.

Observation 8.1 Starting at a time that is $O(\log n)$ after the faults in synchronous networks, and $O\left(\log ^{2} n\right)$ time in asynchronous networks, the trains start delivering only pieces that are stored permanently at nodes in the part.

After the trains stabilize (in the sense described in Observation 8.1), what we want to ensure at this point is that the set of pieces stored in a part (and delivered by the train) includes all the (possibly corrupted) pieces of the form $\mathrm{I}\left(F_{j}(v)\right.$ ), for every $v$ in the part and for every $j$ such that $v$ belongs to a level $j$ fragment. Addressing this, we shall show that the verifier at each node rejects if it does not obtain all the required pieces eventually, whether the partitions are correct or not. Informally, this is done as follows. Recall that each node $v$ knows the set $J(v)$ of levels $j$ for which there exists a fragment of level $j$ containing it, namely, $F_{j}(v)$. Using a delimiter (stored at $v$ ), we partition $J(v)$ to $J_{\text {Top }}(v)$ and $J_{\text {Bottom }}(v)$; where $J_{\text {Top }}(v)$ (respectively, $J_{\text {Bottom }}(v)$ ) is the set of levels $j \in J(v)$ such that $F_{j}(v)$ is top (resp., bottom).

Node $v$ "expects" to receive the claimed information $\hat{\mathrm{I}}\left(F_{j}(v)\right)$ for $j \in J_{\text {Top }}(v)$ (respectively, $j \in J_{\text {Bottom }}(v)$ ) from the train of the part in Top (respectively, Bottom) it belongs to.

Let us now consider the part $P_{\text {Top }} \in$ Top containing $v$. In correct instances, by the way the train operates, it follows that the levels of fragments arriving at $v$ should arrive in a strictly increasing order and in a cyclical manner, that is, $j_{1}<j_{2}<j_{3}<\cdots<j_{a}, j_{1}<j_{2}<\cdots j_{a}, j_{1} \cdots$ (observe that $j_{a}=\ell$ ). Consider the case that the verifier at $v$ receives two consecutive pieces $z_{1} \circ j_{1} \circ \omega_{1}$ and $z_{2} \circ j_{2} \circ \omega_{2}$ such that $j_{2} \leq j_{1}$. The verifier at $v$ then "assumes" that the event $S$ of the arrival of the second piece $z_{2} \circ j_{2} \circ \omega_{2}$ starts a new cycle of the train. Let the set of pieces arriving at $v$ between two consecutive such $S$ events be named a cycle set. To be "accepted" by the verifier at $v$, the set of levels of the fragments arriving at $v$ in each cycle set must contain $J_{\text {Top }}(v)$. It is trivial to verify this in two cycles after the faults cease. (The discussion above is based implicitly on the assumption that each node receives pieces infinitely often; this is guaranteed by the correctness of the train mechanism, assuming that at least one piece is indeed stored permanently in $P_{\text {Top }}$; verifying this assumption is done easily by the root $r\left(P_{\text {Top }}\right)$ of $P_{\text {Top }}$, simply by verifying that $r\left(P_{\text {Top }}\right)$ itself does contain a piece.) Verifying the reception of all the pieces in a part in Bottom is handled very similarly, and is thus omitted. Hence, we can sum up the above discussion as follows: 
Claim 8.2 If the verifier accepts then each node $v$ receives $\hat{I}\left(F_{j}(v)\right.$ ), for every level $j \in J(v)$ (in the time stated in Lemma 7.7), and conversely, if a node does not receive $\hat{I}\left(F_{j}(v)\right.$ ) (in the time stated in Lemma 7.7) then the verifier has rejected.

Let $p(v)$ denote the parent of $v$ in $T$. Recall, that by comparing the data structure of a neighbour $u$ in $T$, node $v$ can know whether $u$ and $v$ belong to the same fragment of level $j$, for each $j$. In particular, this is true for $u$ being the parent of $v$ in $T$. Consider an event $\mathcal{E}(v, p(v), j)$. In case $p(v)$ belongs to the same level $j$ fragment as $v$, node $v$ compares $\hat{\mathrm{I}}\left(F_{j}(v)\right)$ with $\hat{\mathrm{I}}\left(F_{j}(p(v))\right)$, and verifies that these pieces are equal (otherwise, it rejects). By transitivity, if no node rejects, it follows that for every fragment $F \in \mathcal{H}$, we have that $\hat{\mathrm{I}}(F)$ is of the form $z \circ j \circ \omega$, and all nodes in $F$ agree on this. By verifying at the root $r_{F}$ of $F$ that $\operatorname{ID}\left(r_{F}\right)=z$, we obtain the following.

Claim 8.3 If the verifier accepts then:

- The claimed identifiers of the fragments are compatible with the given hierarchy $\mathcal{H}$. In particular, this guarantees that the identifiers of fragments are indeed unique.

- For every $F \in \mathcal{H}$, all the nodes in $F$ agree on the claimed weight of the minimum outgoing edge of fragment $F$, denoted $\hat{\omega}(F)$, and on the identifier of fragment $F$, namely, ID $F)$.

So far, we have shown that each node does receive the necessary information needed for the verifier. Now, finally, we show how to use this information to detect whether this is an MST. Basically, we verify that $\hat{\omega}(F)$ is indeed the minimum outgoing edge $\omega(F)$ of $F$ and that this minimum is indeed the candidate edge of $F$, for every $F \in \mathcal{H}$. Consider a time when $\mathcal{E}(v, u, j)$ occurs. Node $v$ rejects if any of the checks below is not valid.

- C1: If $v$ is the endpoint of the candidate edge $e=(v, u)$ of $F_{j}(v)$ then $v$ checks that $u$ does not belong to $F_{j}(v)$, i.e., that $\operatorname{ID}\left(F_{j}(v)\right) \neq \operatorname{ID}\left(F_{j}(u)\right)$, and that $\hat{\omega}\left(F_{j}(v)\right)=\omega(e)$ (recall, it is already ensured that $v$ knows whether it is an endpoint, and if so, which of its edges is the candidate);

- C2: If $\operatorname{ID}\left(F_{j}(v)\right) \neq \operatorname{ID}\left(F_{j}(u)\right)$ then $v$ verifies that $\hat{\omega}\left(F_{j}(v)\right) \leq \omega((v, u))$.

The following lemma now follows from C1, C2 and Lemma 5.1.

Lemma 8.4 - If by some time $t$, the events $\mathcal{E}(v, u, j)$ occurred for each node $v$ and each neighbour $u$ of $v$ in $G$ and for each level $j$, and the verifier did not reject, then $T$ is an MST of $G$.

- If $T$ is not an MST, then in the time stated in Lemma 7.7 after the faults cease, the verifier rejects.

We are now ready for the following theorem, summarizing Sections 4 to 8

Theorem 8.5 The scheme described in Sections 48 is a correct proof labeling scheme for MST. Its memory complexity is $O(\log n)$ bits. Its detection time complexity is $O\left(\log ^{2} n\right)$ in synchronous networks and $O\left(\Delta \log ^{3} n\right)$ in asynchronous ones. Its detection distance is $O(f \log n)$ if $f$ faults occurred. Its construction time is $O(n)$.

Proof: The correctness and the specified detection time complexity follow from Lemma 8.4 and Claim 8.2 . The space taken by pieces of I stored permanently at nodes (and rotated by the trains) was already shown to be $O(\log n)$ bits. In addition, a node needs some additional $O(\log n)$ bits of memory for the actions described in Section 8 . Similarly, the data-structure at each node and the corresponding 1-proof labeling schemes (that are 
used to verify it) consume additional $O(\log n)$ bits. Finally, for each train, a node needs a constant number of counters and variables, each of logarithmic size. This establishes the required memory size of the scheme.

To show the detection distance, let network $G_{1}$ contain faults. Consider a (not necessarily connected) subgraph $U$ containing every faulty node $v$, every neighbour $u$ of $v$, and the parts, both of Bottom and of Top of $v$ and $u$. First, we claim that no node outside of $U$ will raise an alarm. To see that, assume (by way of contradiction) that some node $w$ outside $U$ does raise an alarm. Now, consider a different network $G_{2}$ with the same sets of nodes and of edges as $G_{1}$. The state of every node in $G_{2} \backslash U$ is exactly the (correct) state of the same node in $G_{1}$. The states of the nodes in $U$ are chosen so that to complete the global configuration to be correct. (Clearly, the configuration can be completed in such a way.) Hence, no node should raise an alarm (since we have shown that our scheme is correct). However, node $w$ in $G_{2}$ receives exactly the same information it receives in $G_{1}$, since it receives only information from nodes in the parts to which it or its neighbours belong. Hence, $w$ will raise an alarm. A contradiction. The detection distance complexity now follows from the fact that the radius of $U$ is $O(f \log n)$. (Informally, this proof also says that non-faulty nodes outside of $U$ are not contaminated by the faulty nodes, since the verification algorithm sends information about the faulty nodes only within $U$.)

The construction time complexity required for the more complex part of the proof labeling scheme, that is, the proof scheme described in Sections 6-8, is dominated by the construction time of the MST algorithm SYNC_MST. This time is shown to be $O(n)$ in Theorem 4.4. The construction time required for the simpler 1-proof labeling scheme described in Section 5 is shown to be linear in Lemma 5.2.

\section{Verification of time lower bound}

We now show that any proof labeling scheme for MST that uses optimal memory must use at least logarithmic time complexity, even when restricted to synchronous networks. The lower bound is derived below from the relatively complex lower bound for 1-proof labeling schemes for MST presented in [54], by a not- too- difficult reduction from that problem. We prove the lower bound on the specific kind of networks used in [54]. These networks are a family of weighted graphs termed $(h, \mu)$-hypertrees. (The name may be misleading; a $(h, \mu)$ hypertrees is neither a tree nor a part of a hyper graph; the name comes from them being a combination of $(h, \mu)$-trees (see also [42, 51]) and hypercubes.) We do no describe these hypertrees here, since we use them, basically, as black boxes. That is, all we need here is to know certain properties (stated below) of these graphs. (We also need to know the lower bound of [54]).

The following two properties of this family were observed in [54]. First, all $(h, \mu)$-hypertrees are identical if one considers them as unweighted. In particular, two homologous nodes in any two $(h, \mu)$-hypertrees have the same identities. Moreover, the components assigned to two homologous graphs in [54] are the same. Hence, the (unweighted) subgraphs $H(G)$ induced by the components of any two $(h, \mu)$-hypertrees are the same. The second property that was observed is that this subgraph $H(G)$ is in fact a (rooted) spanning tree of $G$, the corresponding $(h, \mu)$-hypertree. Another easy observation that can be obtained by following the recursive construction of an $(h, \mu)$-hypertree (see Section 4 of [54]), is that each node in an $(h, \mu)$-hypertree $G$ is adjacent to at most one edge which is not in the tree $H(G)$, and that the root of $H(G)$ is adjacent only to edges in $H(G)$.

Fix an integer $\tau$. Given a $(h, \mu)$-hypertree $G$, we transform $G$ into a new graph $G^{\prime}$ according to the following procedure (see Figures 10 and 11 . We replace every edge $(u, v)$ in $G$ where $\operatorname{ID}(u)<\operatorname{ID}(v)$ with a simple path $P(u, v)$ containing $2 \tau+2$ consecutive nodes, i.e., $P(u, v)=\left(x_{1}, x_{2} \cdots, x_{2 \tau+2}\right)$, where $x_{1}=u$, and $x_{2 \tau+2}=v$. For $i=2, \cdots, 2 \tau+1$, the port number at $x_{i}$ of the port leading to $x_{i-1}$ (respectively, $x_{i+1}$ ) is 1 (resp., 2). The port-number at $x_{1}$ (respectively, $x_{2 \tau+2}$ ) of the port leading to $x_{2}$ (resp., $x_{2 \tau+1}$ ) is the same as the port-number of the port leading from $u$ (resp., $v$ ) to $v$ (resp., $u$ ) in $G$. The weight of the edge $\left(x_{2 \tau+1}, x_{2 \tau+2}\right)$ is the weight of $(u, v)$, that is, $\omega\left(x_{2 \tau+1}, x_{2 \tau+2}\right)=\omega(u, v)$, and the weight of all other edges in $P(u, v)$ is 1 . The identities of 


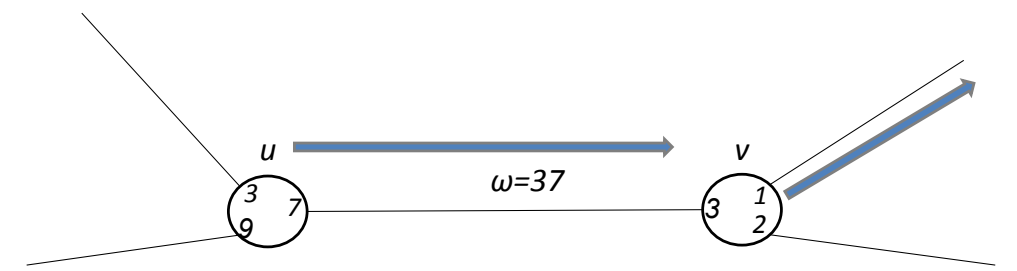

Weight=37

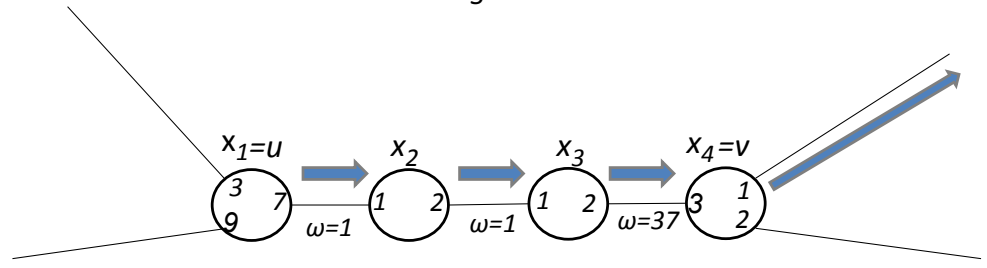

Figure 10: Transforming an edge of $G$ (the upper part) to a path of $G^{\prime}$ (the lower part) for $\tau=1$ and the case that the component of $u$ points at $v$. The component of $v$ points at $v$ 's port 1.

the nodes in the resulted graph are given according to a DFS traversal on $G^{\prime}$. We now describe the component of each node in $G^{\prime}$. Let $(u, v)$ be an edge in $G$ and let $P(u, v)=\left(x_{1}, x_{2} \cdots, x_{2 \tau+2}\right)$ be the corresponding path in $G^{\prime}$, where $x_{1}=u$ and $x_{2 \tau+2}=v$ (here we do not assume necessarily that $\operatorname{ID}(u)<\operatorname{ID}(v)$ ). Consider first the case that in the graph $G$, the edge $(u, v)$ belongs to the tree $H(G)$. Assume without loss of generality that the component of $u$ in $G$ points at $v$. For each $i=1,2, \cdots, 2 \tau+1$, we let the component at $x_{i}$ point at $x_{i+1}$ (the component at $x_{2 \tau+2}$ is the same as the component of $v$ in $\left.G\right)$. Consider now the case that $(u, v)$ does not belong to $H(G)$. In this case, for $i=2,3, \cdots, \tau+1$, we let the component at $x_{i}$ point at $x_{i-1}$ (the component at $x_{1}=u$ in $G^{\prime}$ is the same as the component of $u$ in $G$ ) and for $i=\tau+2, \tau+3, \cdots, 2 \tau+1$, we let the component at $x_{i}$ point at $x_{i+1}$ (similarly, the component at $x_{2 \tau+2}$ is the same as the component of $v$ in $G$ ).

By this construction of $G^{\prime}$, we get that the subgraph $H\left(G^{\prime}\right)$ induced by the components of $G^{\prime}$ is a spanning tree of $G^{\prime}$, and it is an MST of $G^{\prime}$ if and only if $H(G)$ is an MST of $G$. Let $\mathcal{F}(h, \mu, \tau)$ be the family of all weighted graphs $G^{\prime}$ obtained by transforming every $(h, \mu)$-hypertree $G$ into $G^{\prime}$ using the method explained above.

Lemma 9.1 If there exists a proof labeling scheme for $M S T$ on the family $\mathcal{F}(h, \mu, \tau)$ with memory complexity $\ell$ and detection time $\tau$ then there exists a 1-proof labeling scheme (a proof labeling scheme in the sense of [54]) for the MST predicate on the family of $(h, \mu)$-hypertrees with label size $O(\tau \ell)$.

Proof: Let $\left(\mathcal{M}^{\prime}, \mathcal{V}^{\prime}\right)$ be a proof labeling scheme for MST and the family $\mathcal{F}(h, \mu, \tau)$ with memory complexity $\ell$ and detection time $\tau$. We describe now a 1-proof labeling scheme $(\mathcal{M}, \mathcal{V})$ for the MST predicate on the family of $(h, \mu)$-hypertrees. Let $G$ be an $(h, \mu)$-hypertree that satisfies the MST predicate. We first describe the labels assigned by the marker $\mathcal{M}$ to the nodes on $G$. In this lemma, we are not concerned with the time needed for actually assigning the labels using a distributed algorithm, hence, we describe the marker $\mathcal{M}$ as a centralized algorithm and not as a distributed one. (We note that this is consistent with the model of [54] that considers only centralized marker algorithms.)

The marker $\mathcal{M}$ transforms $G$ to $G^{\prime}$. Observe that $G^{\prime}$ must also satisfy the MST predicate. $\mathcal{M}$ labels the nodes of $G^{\prime}$ using the marker $\mathcal{M}^{\prime}$. Note that any label given by the marker $\mathcal{M}^{\prime}$ uses at most $\ell$ bits. Given a node $u \in G$, let $e_{1}(u)$ be the edge not in the tree $H(G)$ that is adjacent to $u$ (if one exists) and let $e_{2}(u)$ be the edge 


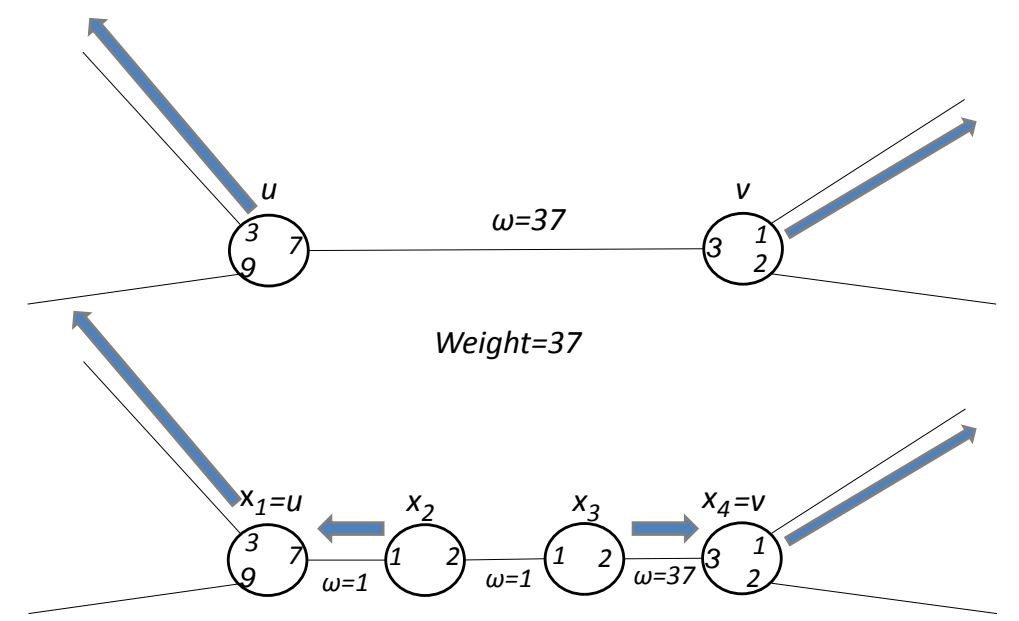

Figure 11: The case that the component of $u$ points at $u$ 's port 3 that does not lead to $v$, and the component of $v$ points at $v$ 's port 1 that does not lead to $u$.

in $H(G)$ leading from $u$ to its parent in $H(G)$ (if one exists). Let $P_{1}(u)=\left(w_{1}, w_{2} \cdots, w_{2 \tau+2}\right)$ be the path in $G^{\prime}$ corresponding to $e_{1}(u)$. If $u$ is not the root of $H(G)$ then $e_{2}(u)$ exists and let $P_{2}(u)=\left(y_{1}, y_{2} \cdots, y_{2 \tau+2}\right)$ be the path in $G^{\prime}$ corresponding to $e_{2}(u)$, where $y_{1}=u$ and $y_{2 \tau+2}$ is the parent of $u$. For the root $r$ of $H(G)$, let $P_{2}(r)$ be simply $(r)$. If $u$ is not the root of $H(G)$ then for each $i \in\{1,2, \cdots, 2 \tau+1\}$, the marker $\mathcal{M}$ copies the labels $\mathcal{M}^{\prime}\left(y_{i}\right)$ and $\mathcal{M}^{\prime}\left(w_{i}\right)$ into the $i$ 'th field in the label $\mathcal{M}(u)$. (Note that the labels $\mathcal{M}^{\prime}\left(w_{i}\right)$ are copied in the labels given by $\mathcal{M}$ to both end-nodes of $e_{1}(u)$.) If $r$ is the root of $H(G)$ then $e_{2}(r)$ does not exist and actually, also $e_{1}(r)$ does not exist, as $r$ is not adjacent to any edge not in $H(G)$. The marker $\mathcal{M}$ simply copies the label $\mathcal{M}^{\prime}\left(r^{\prime}\right)$ into the label $\mathcal{M}(r)$, where $r^{\prime}$ is the corresponding node of $r$ in $G^{\prime}$.

In the model of proof labeling schemes in [54], the verifier $\mathcal{V}$ at a node $u \in G$ can look at the labels of all nodes $v$ such that $(u, v)$ is an edge of $G$. In particular, it sees the labels assigned by $\mathcal{M}^{\prime}$ to all nodes in $G^{\prime}$ at distance at most $2 \tau$ from its corresponding node $u^{\prime}$ in $G^{\prime}$. Let $B_{\tau}\left(u^{\prime}\right)$ be the set of nodes at distance at most $\tau$ from $u^{\prime}$ in $G^{\prime}$. We let the verifier $\mathcal{V}$ at $u$ simulate the operations of the verifier $\mathcal{V}^{\prime}$ at each node in $B_{\tau}\left(u^{\prime}\right)$-this can be achieved as the information in the 1-neighbourhood of $u$ (in $G$ ) contains the information in the $\tau$-neighbourhood of $G^{\prime}$ of any node in $B_{\tau}\left(u^{\prime}\right)$. Finally, we let $\mathcal{V}(u)=1$ if and only if $\mathcal{V}^{\prime}(x)=1$ for all $x \in B_{\tau}\left(u^{\prime}\right)$. It can be easily observed that $(\mathcal{M}, \mathcal{V})$ is indeed a 1-proof labeling scheme for the family of $(h, \mu)$-hypertrees. Moreover, each label assigned by the marker $\mathcal{M}$ uses $O(\tau \ell)$ bits. (Note that the model in [54] restricts only the sizes of the labels and not the memory size used by the verifier.) This completes the proof of the lemma.

Corollary 9.2 Fix a positive integer $\tau=O(\log n)$. The memory complexity of any proof labeling scheme for $\mathcal{F}(n)$ with detection time $\tau$ is $\Omega\left(\frac{\log ^{2} n}{\tau}\right)=\Omega(\log n)$. (Recall $\mathcal{F}(n)$ represents all connected undirected weighted graphs.)

Proof: In [54] we showed that the label size of any proof labeling scheme for the MST predicate and the family of $(\log n, n)$-hypertrees is $\Omega\left(\log ^{2} n\right)$ bits. The claim now follows by combining the previous lemma together with the fact that the number of nodes in a graph $G^{\prime} \in \mathcal{F}(\log n, n, \tau)$ is polynomial in $n$. 


\section{The self-stabilizing MST construction algorithm}

We use a transformer that inputs a non-self-stabilizing algorithm and outputs a self-stabilizing one. For simplicity, we first explain how to use the transformer proposed in the seminal paper of Awerbuch and Varghese [15] (which utilizes the transformer of its companion paper [13] as a black box). This already yields a self-stabilizing MST algorithm with $O(n)$ time and $O(\log n)$ memory per node. Later, we refine that transformer somewhat to add the property that the verification time is of $O\left(\log ^{2} n\right)$ in a synchronous network, or $O\left(\min \left\{\Delta \log ^{3} n, n\right\}\right)$ in an asynchronous one. We then also establish the property that if $f$ faults occur, then each fault is detected within its $O(f \log n)$ neighbourhood.

The Resynchronizer of [15] inputs a non-stabilizing synchronous input/output algorithm 4 [ $\Pi$ whose running time and memory size are some $T_{\Pi}$ and $S_{\Pi}$, respectively. Another input it gets is $\hat{D}$, which is an upper bound on the actual diameter $D$ of the network. It then yields a self-stabilizing version whose memory size is $O\left(S_{\Pi}+\log n\right)$ and whose time complexity is $O\left(T_{\Pi}+\hat{D}\right)$.

For our purposes, to have the Resynchronizer yield our desired result, we first need to come up with such a bound $\hat{D}$ on the diameter. (Recall that we do not assume that $D$, or even $n$, are known). Second, the result of the Resynchronizer of [15] is a synchronous algorithm, while we want an algorithm that can be executed in an asynchronous network. Let us describe how we bridge these two gaps.

We use known self-stabilizing protocols [1, 28] to compute $D$, the diameter of the network, in time $O(n)$, using $O(\log n)$ bits of memory per node. We use this computed $D$ as the desired $\hat{D}$. Note that at the time that [15] was written, the only algorithm for computing a good bound (of $n$ ) on the diameter with a bounded memory had time complexity $\Theta\left(n^{2}\right)[3]$.

To bridge the second gap, of converting the resulting self-stabilizing algorithm for an asynchronous network, we use a self-stabilizing synchronizer that transforms algorithms designed for synchronous networks to function correctly in asynchronous ones. Such a synchronizer was not known at the time that [15] was written, but several are available now. The synchronizer of [10, 11] was first described as if it needs unbounded memory. However, as is stated in [10], this synchronizer is meant to be coupled with a reset protocol to bound the memory. That is, to have a memory size of $O(\log n)$ and time $O(n)$, it is sufficient to use a reset protocol with these complexities. We use the reset protocol of [13]. Similarly, this reset protocol is meant to be coupled with a self-stabilizing spanning tree construction algorithm. The complexities of the resulting reset protocol are dominated by those of the spanning tree construction. We plug in some spanning tree algorithm with the desired properties (such as [1, 28]) whose memory size and time complexities are the desired $O(\log n)$ and $O(n)$ in asynchronous networks, respectively. (It is easy to improve the time to $O(D)$ in synchronous networks.) This yields the desired reset protocol, and, hence, the desired synchronizer protoco 5

Let us sum up the treatment of the first two gaps: thanks to some new modules developed after [15], one can now use the following version of the main result of [15].

Theorem 10.1 Enhanced Awerbuch-Varghese Theorem, (EAV): Assume we are given a distributed algorithm $\Pi$ to compute an input/output relation. Whether $\Pi$ is synchronous or asynchronous, let $T_{\Pi}$ and $S_{\Pi}$ denote $\Pi$ 's time complexity and memory size, respectively, when executed in synchronous networks. The enhanced Resynchronizer compiler produces an asynchronous (respectively, synchronous) self-stabilizing algorithm whose memory size is $O\left(S_{\Pi}+\log n\right)$ and whose time complexity is $O\left(T_{\Pi}+n\right)\left(\right.$ resp., $\left.O\left(T_{\Pi}+D\right)\right)$.

The EAV theorem differs from the result in [15] by (1) addressing also asynchronous algorithms, and (2) basing

\footnotetext{
${ }^{4} \mathrm{An}$ input/output algorithm is one whose correctness requirement can be specified as a relation between its input and its output.

${ }^{5}$ An alternative synchronizer can be based on the one of [19], again, coupled with some additional known components, such as a module to compute $n$.
} 
the time complexity on the actual values of $n$ and $D$ of the network rather than on an a-priori bound $\hat{D}$ that may be arbitrarily larger than $D$ or $n$.

Recall from Theorem 4.4 that in synchronous networks, algorithm SYNC_MST constructs an MST in $O(n)$ time and using $O(\log n)$ memory bits per node. Hence, plugging in algorithm SYNC_MST as $\Pi$ yields the following theorem.

Theorem 10.2 There exists a self-stabilizing MST construction algorithm that can operate in an asynchronous environment, runs in $O(n)$ time and uses $O(\log n)$ bits of memory per node.

\subsection{Obtaining fast verification}

The Resynchronizer compiler performs iterations forever. Essentially, the first iteration is used to compute the result of $\Pi$, by executing $\Pi$ plus some additional components needed for the self-stabilization. Each of the later iterations is used to check that the above result is correct. For that, the Resynchronizer executes a checker. If the result is not correct, then the checker in at least one node "raises an alarm". This, in effect, signals the Resynchronizer to drop back to the first iteration. Let us term such a node a detecting node. Our refinement just replaces the checker, interfacing with the original Resynchronizer by supplying such a detecting node.

We should mention that the original design in [15] is already modular in allowing such a replacement of a checker. In fact, two alternative such checkers are studied in [15]. The first kind of a checker is $\Pi$ itself. That is, if $\Pi$ is deterministic, then, if executed again, it must compute the same result again (this is adjusted later in [15] to accommodate randomized protocols). This checker functions by comparing the result computed by $\Pi$ in each "non-first" iteration to the result it has computed before. If they differ, then a fault is detected. The second kind of a checker is a local checker of the kinds studied in [3, 13] or even one that can be derived from local proofs [54, 55]. That is, a checker whose time complexity is exactly 1 . When using this kind of a checker, the Resynchronizer uses one iteration to execute $\Pi$, then the Resynchronizer executes the checker repeatedly until a fault is detected. It was argued in [15] that the usage of such a checker (of time complexity exactly 1) is easy, since such a checker self-stabilizes trivially. We stress that it was later shown that such a checker (whose time complexity is 1 ) must use $\Omega\left(\log ^{2} n\right)$ bits [55]. Hence, plugging such a checker into the Resynchronizer compiler cannot yield an optimal memory self-stabilizing algorithm.

The door was left open in [15] for additional checkers. It was in this context that they posed the open problem of whether MST has a checker which is faster than MST computation, and still uses small memory. (Recall that Theorem 8.5 answers the open problem in the affirmative.)

We use a self-stabilizing verifier (of a proof labeling scheme) as a checker. That is, if a fault occurs, then the checker detects it, at least in one node, regardless of the initial configuration. Such nodes where the fault is detected serve as the detecting nodes used above by the Resynchrnonizer. The following theorem differs from the EAV theorem by stating that the final protocol (resulting from the transformation) also enjoys the good properties of the self-stabilizing verifier. I.e., if the self-stabilizing verifier has a good detection time and good detection distance, then, the detection time and distance of the resulting protocols are good too.

Theorem 10.3 Suppose we are given the following:

- A distributed algorithm $\Pi$ to compute an input/output relation $R$. Whether $\Pi$ is synchronous or asynchronous, let $T_{\Pi}$ and $S_{\Pi}$ denote $\Pi$ 's time complexity and memory size, when executed in synchronous networks.

- An asynchronous (respectively, synchronous) proof labeling scheme $\Pi^{\prime}$ for verifying $R$ with memory size $S_{\Pi^{\prime}}$, 
whose verifier self-stabilizes with verification time and detection distance $t_{\Pi^{\prime}}$ and $d_{\Pi^{\prime}}$, and whose construction time (of the marker) is $T_{\Pi^{\prime}}$.

Then, the enhanced Resynchronizer produces an asynchronous (resp., synchronous) self-stabilizing algorithm whose memory and time complexities are $O\left(S_{\Pi}+S_{\Pi^{\prime}}+\log n\right)$ and $O\left(T_{\Pi}+T_{\Pi^{\prime}}+t_{\Pi^{\prime}}+n\right)\left(\right.$ resp., $O\left(T_{\Pi}+T_{\Pi^{\prime}}+\right.$ $\left.\left.t_{\Pi^{\prime}}+D\right)\right)$, and whose verification time and detection distance are $t_{\Pi^{\prime}}$ and $d_{\Pi^{\prime}}$.

Proof: The proof relies heavily on the Resynchronizer compiler given by the EAV theorem (Theorem 10.1). This Resynchronizer receives as input the following algorithm $\Pi^{\prime \prime}$, which is not assumed to be neither self-stabilizing nor asynchronous. Specifically, algorithm $\Pi^{\prime \prime}$ first constructs the relation $R$ using algorithm $\Pi$ and, subsequently, executes the marker algorithm of the proof labeling scheme $\Pi^{\prime}$.

The resulted Resynchronizer (when executing together with the algorithm $\Pi^{\prime \prime}$ it transforms) is a detection based self-stabilizing algorithm (see the explanation of the detection time and distance in Section 2.5). It executes algorithm $\Pi^{\prime \prime}$ for a set amount of time (here, counting the time using the self-stabilizing synchronizer) and then puts all the nodes in an output state, where it uses the self-stabilizing verifier of the proof labeling scheme $\Pi^{\prime}$ to check. (Recall, in contrast to the marker algorithm, the verifier algorithm of $\Pi^{\prime}$ is assumed to be self-stabilizing.) The detection time and the detection distance of the combined algorithm thus follow directly from the detection time and the detection distance of the proof labeling scheme $\Pi^{\prime}$. This concludes the proof of the theorem.

Now, as algorithm $\Pi$, we can plugged in Theorem 10.3 the MST construction algorithm SYNC_MST, that uses optimal memory size and runs in $O(n)$ time. Furthermore, two possible proof labeling schemes that can be plugged in Theorem 10.3 as $\Pi^{\prime}$ are the schemes of [54, 55]. Both these schemes use $O\left(\log ^{2} n\right)$ memory size. Since their detection time is 1 , they stabilize trivially. The corresponding distributed markers are simplified versions of the marker of the proof labeling scheme given of the current paper, and hence their construction time is $O(n)$. Hence, plugging either one of these schemes as $\Pi^{\prime}$ yields the following.

Corollary 10.4 There exists a self-stabilizing MST algorithm with $O\left(\log ^{2} n\right)$ memory size and $O(n)$ time. Moreover, its detection time is 1 and its detection distance is $f+1$.

Finally, by plugging to the Resynchronizer given in Theorem 10.3 , the construction algorithm SYNC_MST as $\Pi$ and our optimal memory proof labeling scheme mentioned in Theorem 8.5 as $\Pi^{\prime}$, we obtain the following.

Theorem 10.5 There exists a self-stabilizing MST algorithm that uses optimal $O(\log n)$ memory size and $O(n)$ time. Moreover, its detection time complexity is $O\left(\log ^{2} n\right)$ in synchronous networks and $O\left(\Delta \log ^{3} n\right)$ in asynchronous ones. Furthermore, its detection distance is $O(f \log n)$.

\subsection{Combining self stabilzing algorithms}

The algorithm in this paper is composed of multiple modules (Figures 12 and 13). Some of them are self stabilizing, and some are not. When composing self stabilizing algorithms together, the result may not be self stabilizing, so one should take care [34]. We have claimed the stabilization of composite programs throughout this paper. For the sake of completeness, let us go over all the components here once again, to recall that their composition self stabilizes in spite of the composition.

The main composition is that of the transformer algorithm of Awerbuch and Varghese [15] together with a checking scheme. The way to perform this composition, as well as its correctness, have been established in [66] (as well as in [15]). See Theorem 6.1 in [15] and Theorem 9.3.2 in [66]. 


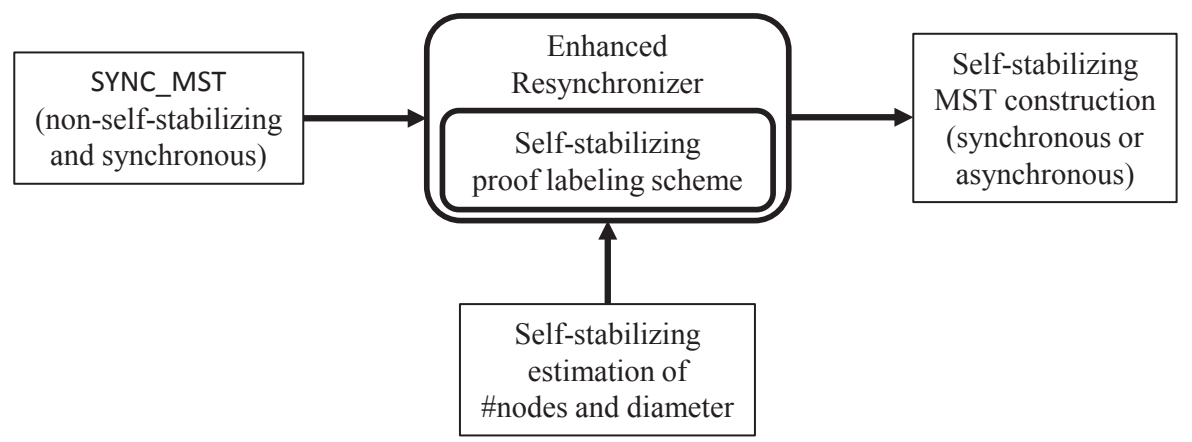

Figure 12: Structure of the self-stabilizing (synchronous or asynchronous) MST construction algorithm obtained by the enhanced Resynchronizer.

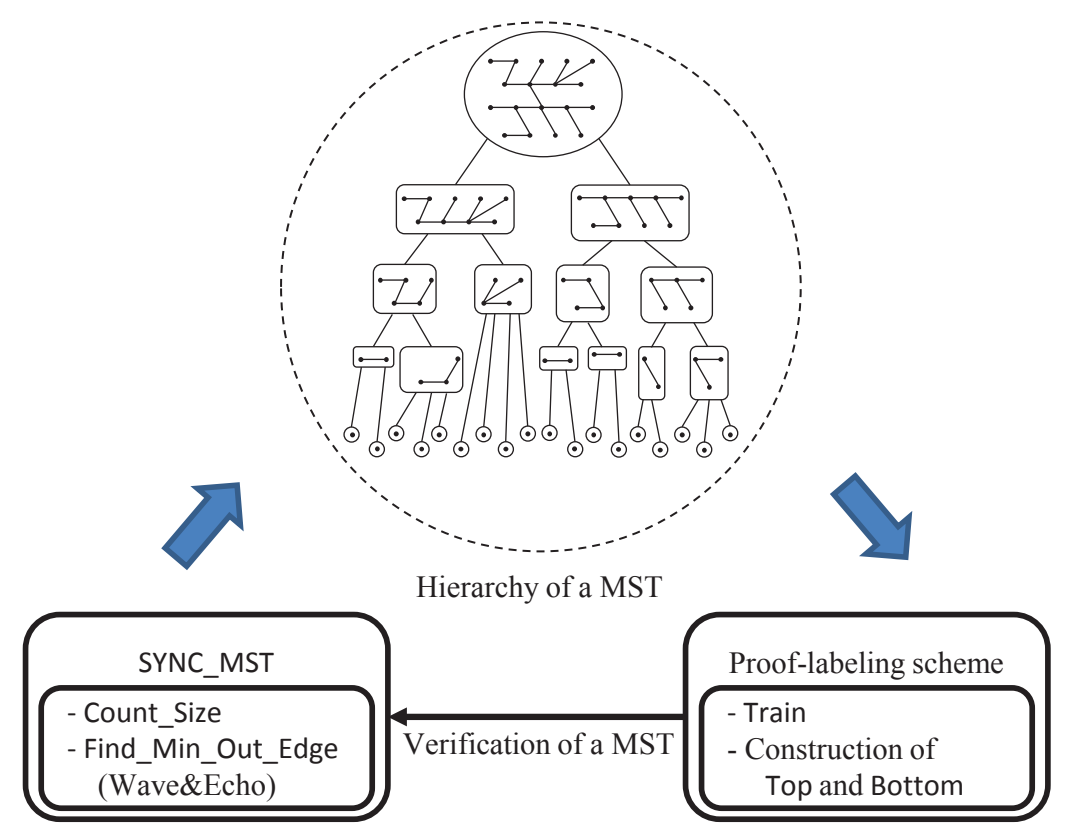

Figure 13: Algorithm SYNC_MST, mainly consisting of algorithms Count_Size and Find_Min_Out_Edge, induces the hierarchy of a MST. From the hierarchy the proof-labeling-scheme, mainly consisting of the trains and the construction of partitions Bottom and Top, produces a marker and a verifier. 
A synchronizer uses, as an input, the number of nodes and the value of the diameter computed by other algorithms. Here the correctness follows easily from the "fair combination" principle of [34, 64]. That is, the algorithms computing these values do not use inputs from the other algorithms in the composition. Moreover, their outputs stabilize to the correct values at some points (from their respective proofs, that do not depend on assumptions in other algorithms). From that time on, their values are correct.

The tree construction itself is not supposed to be self stabilizing for the transformer scheme of [15]. This is also the case with the marker algorithm, since the MST construction algorithm and the marker together constructs a data structure to be verified. (Recall that verifying the MST alone is costly [53]; hence the idea is to construct a "redundant" representation of the MST, containing the MST plus the proof labels, such that verifying this redundant data structure is easier).

It is left to argue that the verifier on its own self stabilizes, in spite of the fact that it is composed of several components. Recall that the output of the verifier is a logical AND of several verifiers. That is, if either the verifier for the scheme for the Well-Forming property (Sections 4 and 5) or the verifier for the scheme for the Minimality property (Sections 6, 7, and 8) outputs "no", then the combined (composed together) verifier outputs no. Hence, the different schemes do not interfere with each other. If all of them are self stabilizing, then the composition is self stabilizing. In particular, the scheme for verifying the Well-Forming property runs in one time unit repeatedly. As observed by [15], such a verifier is necessarily self stabilizing. It is then left to show that the verifier for the Minimality property self stabilizes.

Note that Section 6 describes a part of the marker, devoted to the scheme for verifying the Minimality property. Recall that the marker is not required to self stabilize. Section 7 describes the trains process which is composed of two parts: the convergecast of the information to a part's root, and its broadcast from the root. The second process (the broadcast) inputs (at the root) the results of the first process, but not vice versa. Hence, clearly, the composition self stabilizes as above (that is, after the first process eventually stabilizes, the second process will eventually stabilize too). The pieces of information carried by the train are then used by each node to compare information with its neighbours (in Section 7.2) and by the part root (in Section 5). Again, the flow of information between modules is one way. That is, from the train process to the computations by each node and by the root. After the trains stabilizes, so does the rest, eventually. (The later computations also input the output of the module computing the number $n$ of nodes in the network; again, the flow of information is only unidirectional, and hence the verifier does stabilize after the $n$ computation stabilizes).

Comment 10.1 Using later synchronizers: As explained in Section 10, for simplicity of the presentation we prefer using the synchronizer and the reset protocols built in the scheme of [15], since the proof of their composition is already covered in [15] 66]. For those who prefer using the later synchronizers and reset protocols we mentioned, e.g., [11], the composition would remain self stabilizing even if we use those. The correction of this statement has essentially been established in those synchronizers papers. That is, they presented synchronizers such that they can take any algorithm intended to run over a synchronous network, compose with it, and have it run correctly (and in a self-stabilizing manner) in an asynchronous network. The same holds also for self stabilizing reset protocols.

For the sake of completeness, let us recall, nevertheless, why this composition is correct. For the synchronizer to work, it needs a certain output from the algorithm. This output is TRIVIAL. That is, a SYNCHRONOUS algorithm at a node at a pulse acts as follows. It receives messages from ALL the neighbours (or at least a statement that no message is going to arrive from a specific neighbour), and then processes a message from each neighbour. Then it is ready for the next pulse.

Thus, the synchronizer needs to know (1) when did the algorithm receive messages from all the neighbours. For this purpose, the synchronizer receives the messages on the algorithm's behalf, and when it receives all of them (or notifications that no messages will be sent), it passes all of them to the algorithm together, which, in turn, 
processes all of these messages together. The algorithm needs then to tell the synchronizer that it has finished processing the messages. If this processing generates messages to be sent to neighbours, the algorithm needs to give these new messages to the synchronizer to send them on the algorithm's behalf. (This is done so that if there is a neighbour $u$ to which the node does not send a message in the current pulse, the synchronizer will send a "dummy" message, saying that no message will arrive.)

The analysis of the synchronizers (in the papers that presented self stabilizing synchronizers, e.g., [11]) were base on the rather obvious observation regarding the correctness of this trivial information for any "reasonable" algorithm, starting from the second round. That is, it is not assumed that the computation or the messages are correct. What is assumed by the synchronizers is just the fact that the algorithm computed already the messages from the previous round (and is giving the synchronzer the resulting messages). Obviously, this assumption holds for our algorithm too, so we can rely on the results of the papers where the synchronizers were designed. 


\section{References}

[1] Y. Afek and A. Bremler-Barr. Self-stabilizing unidirectional network algorithms by power supply. Chicago Journal of Theoretical Computer Science: 111-120 (1997).

[2] Y. Afek, S. Kutten, and M. Yung. Memory-efficient self stabilizing protocols for general networks. WDAG (renamed DISC), 15-28 (1991).

[3] Y. Afek, S. Kutten, and M. Yung. The local detection paradigm and its application to self-stabilization. Theoretical Computer Science, 186(1-2), 199-229 (1997).

[4] S. Aggarwal and S. Kutten. Time optimal self-stabilizing spanning tree algorithms. FSTTCS, 400-410 (1993).

[5] G. Antonoiu and P. Srimani. Distributed Self-Stabilizing Algorithm for Minimum Spanning Tree Construction. Euro-Par 1300, 480-487 (1997).

[6] A. Arora and M. G. Gouda. Distributed reset. IEEE Transactions on Computers, 43(9), 1026-1038 (1994).

[7] B. Awerbuch. A new distributed depth first search algorithm. Information Processing Letters, 20(3), 147150 (1985).

[8] B. Awerbuch. Complexity of network synchronization. JACM, 32(4), 804-823 (1985).

[9] B. Awerbuch. Optimal distributed algorithms for minimum weight spanning tree, counting, leader election and related problems. STOC, 230-240 (1987).

[10] B. Awerbuch, S. Kutten, Y. Mansour, B. Patt-Shamir, and G. Varghese. Time optimal self-stabilizing synchronization. STOC, 652-661 (1993).

[11] B. Awerbuch, S. Kutten, Y. Mansour, B. Patt-Shamir, and G. Varghese. A time-optimal self-stabilizing synchronizer using a phase clock. IEEE Transactions on Dependable and Secure Computing, 4(3), 180190 (2007).

[12] B. Awerbuch and R. Ostrovsky. Memory-efficient and self-stabilizing network RESET. PODC, 254-263 (1994).

[13] B. Awerbuch, B. Patt-Shamir, and G. Varghese. Self-stabilization by local checking and correction. FOCS, 268-277 (1991).

[14] B. Awerbuch, B. Patt-Shamir, G. Varghese, and S. Dolev. Self-stabilization by local checking and global reset. WDAG: 326-339 (1994).

[15] B. Awerbuch and G. Varghese. Distributed program checking: a paradigm for building self-stabilizing distributed protocols. FOCS: 258-267 (1991).

[16] Y. Azar, S. Kutten, and B. Patt-Shamir. Distributed error confinement. ACM Transactions on Algorithms, 6(3), No. 48 (2010).

[17] L. Blin, S. Dolev, M. G. Potop-Butucaru, and S. Rovedakis. Fast self-stabilizing minimum spanning tree construction. DISC, 480-494 (2010).

[18] L. Blin, M. Potop-Butucaru, S. Rovedakis, and S. Tixeuil. A new self-stabilizing minimum spanning tree construction with loop-free property. DISC, 407-422 (2009). 
[19] C. Boulinier, F. Petit, and V. Villain. When graph theory helps self-stabilization. PODC, 150-159 (2004).

[20] A. Bui, A. K. Datta, F. Petit, and V. Villain. Snap-stabilization and PIF in tree networks. Distributed Computing, 20(1), 3-19 (2007).

[21] E. J. H. Chang, Echo algorithms: depth parallel operations on general graphs. IEEE Transactions on Software Engineering, 8(4), 391-401 (1982).

[22] I. Chlamtac and S. Kutten. Tree-based broadcasting in multihop radio networks. IEEE Transactions on Computers, 36(10), 1209-1223 (1987).

[23] Z. Collin and S. Dolev. Self-stabilizing depth-first search. Information Processing Letters, 49(6), 297-301 (1994).

[24] A. Cournier, F. Petit, V. Villain, and A. K. Datta. Self-stabilizing PIF algorithm in arbitrary rooted networks. ICDCS, 91-98 (2001).

[25] Y. K. Dalal. A distributed algorithm for constructing minimal spanning trees. IEEE Transactions on Software Engineering, 13(3), 398-405 (1987).

[26] A. Das Sarma, S. Holzer, L. Kor, A. Korman, D. Nanongkai, G. Pandurangan, D. Peleg, and R. Wattenhofer. Distributed verification and hardness of distributed approximation. SIAM Journal on Computing 41(5), 1235-1265 (2012).

[27] A. K. Datta, L. L. Larmore, and H. Piniganti. Self-stabilizing leader election in dynamic networks. SSS, 35-49 (2010).

[28] A. K. Datta, L. L. Larmore, and P. Vemula. Self-stabilizing leader election in optimal space. SSS, 109-123 (2008).

[29] E. W. Dijkstra. Self-stabilizing systems in spite of distributed control. CACM, 17(11), 643-644 (1974).

[30] B. Dixon, M. Rauch, and R. E. Tarjan. Verification and sensitivity analysis of minimum spanning trees in linear time. SIAM Journal on Computing, 21(6), 1184-1192 (1992).

[31] B. Dixon and R. E. Tarjan. Optimal parallel verification of minimum spanning trees in logarithmic time. Algorithmica, 17(1), 11-18 (1997).

[32] S. Dolev. Self-stabilization. MIT Press (2000).

[33] S. Dolev, M. Gouda, and M. Schneider. Requirements for silent stabilization. Acta Informatica, 36(6), 447-462 (1999).

[34] S. Dolev, A. Israeli, and S. Moran. Self-stabilization of dynamic systems assuming only read/write atomicity. Distributed Computing, 7(1), 3-16 (1994).

[35] S. Even. Graph Algorithms. Computer Science Press (1979).

[36] P. Fraigniaud and C. Gavoille. Routing in trees. ICALP, 757-772 (2001).

[37] P. Fraigniaud, A. Korman, and D. Peleg. Local distributed decision. FOCS, 708-717 (2011).

[38] P. Fraigniaud, A. Korman, M. Parter, and D. Peleg. Randomized Distributed Decision. DISC, 371-385 (2012). 
[39] P. Fraigniaud, M.M. Halldorsson and A. Korman. On the Impact of Identifiers on Local Decision. OPODIS (2012).

[40] R. G. Gallager, P. A. Humblet, and P. M. Spira. A distributed algorithm for minimum-weight spanning trees. ACM Transactions on Programming Languages and Systems, 5(1), 66-77 (1983).

[41] J. Garay, S. Kutten, and D. Peleg. A sub-linear time distributed algorithm for minimum-weight spanning trees. FOCS, 659-668 (1993).

[42] C. Gavoille, D. Peleg, S. Pérennes, and R. Raz. Distance labeling in graphs. Journal of Algorithms, 53(1), 85-112 (2004).

[43] S. Ghosh, A. Gupta, T. Herman, and S. V. Pemmaraju. Fault-containing self-stabilizing algorithms. PODC, 45-54 (1996).

[44] Goemans, Michel X."Minimum bounded degree spanning trees." In Foundations of Computer Science, 2006. FOCS’06. 47th Annual IEEE Symposium on, pp. 273-282. IEEE, 2006.

[45] O. Goldreich and L. Shrira. Electing a leader in a ring with link failures. Acta Informatica, 24(1), 79-91 (1987).

[46] M. Göös and J. Suomela. Locally checkable proofs. PODC, 159-168 (2011).

[47] S. K. S. Gupta and P. K. Srimani. Self-stabilizing multicast protocols for ad hoc networks. Journal of Parallel and Distributed Computing, 63(1), 87-96 (2003).

[48] L. Higham and Z. Liang. Self-stabilizing minimum spanning tree construction on message passing networks. DISC, 194-208 (2001).

[49] A. Israeli and M. Jalfon. Token management schemes and random walks yield self stabilizing mutual exclusion. PODC, 119-132 (1990).

[50] G. M. Jayaram and G. Varghese. The fault span of crash failures. JACM, 47(2), 244-293 (2000).

[51] M. Katz, N. A. Katz, A. Korman, and D. Peleg. Labeling schemes for flow and connectivity. SIAM Journal on Computing, 34, 23-40 (2004).

[52] S. Katz and K. J. Perry. Self-stabilizing extensions for message-passing systems. Distributed Computing, 7(1), 17-26 (1993).

[53] L. Kor, A. Korman, and D. Peleg. Tight bounds for distributed MST verification. STACS, 69-80 (2011).

[54] A. Korman and S. Kutten. Distributed verification of minimum spanning trees. Distributed Computing, 20(4), 253-266 (2007).

[55] A. Korman, S. Kutten, and D. Peleg. Proof labeling schemes. Distributed Computing, 22(4), 215-233 (2010).

[56] A. Korman and D. Peleg. Compact separator decomposition for dynamic trees and applications. Distributed Computing, 21(2), 141-161 (2008).

[57] S. Kutten and D. Peleg. Fast distributed construction of k-dominating sets and applications. PODC, 238249 (1995). 
[58] Z. Lotker, B. Patt-Shamir, and D. Peleg. Distributed MST for constant diameter graphs. Distributed Computing, 18(6), 453-460 (2006).

[59] J. McQuillan, I. Richer, and E. Rosen. The New Routing Algorithm for the ARPANET. IEEE Transactions on Communications, 28(5), 711-719 (1980).

[60] M. Naor and L. Stockmeyer. What can be computed locally? STOC, 184-193 (1993).

[61] D. Peleg. Distributed Computing: A Locality-sensitive Approach. SIAM (2000).

[62] D. Peleg and V. Rubinovich. A near-tight lower bound on the time complexity of distributed minimumweight spanning tree construction. SIAM Journal on Computing, 30(5), 1427-1442 (2000).

[63] A. Segall. Distributed network protocols. IEEE Transactions on Information Theory, 29(1), 23-34 (1983).

[64] F. A. Stomp. Structured design of self stabilizing programs. Proc. IEEE 2nd Israeli Symp. on Theory of Comput. Systems: 167-176 (1993).

[65] R. E. Tarjan. Applications of path compression on balanced trees. JACM, 26(4), 690-715 (1979).

[66] G. Varghese. Self-stabilization by local checking and correction. PhD dissertation, Laboratory for Computer Science, Massachusetts Inst. of Tech nology (1992). 